\title{
B ULLET IN
}

OF THE

\author{
UNITED STATES
}

\section{GEOLOGICAL SURVEY}

\section{No. 176}

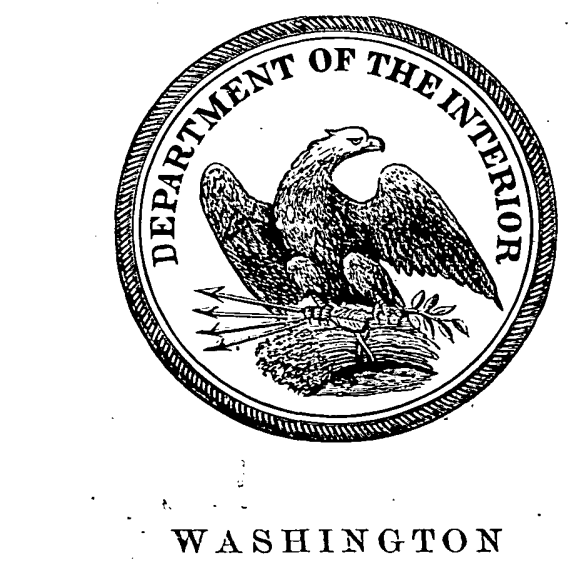

GOVERNMEN'T PRINTING OFFICE

- 1900 

UNITED STA'TES (IEOTOGICAL SURVEY CHAJLES T. WALCOT'L, DRREC'OR

\section{SOME PRINCIPLES AND METHODS}

\section{ROCK A N A Y S I S}

BY

WILLIAM FRANCIS HILLEBRAND

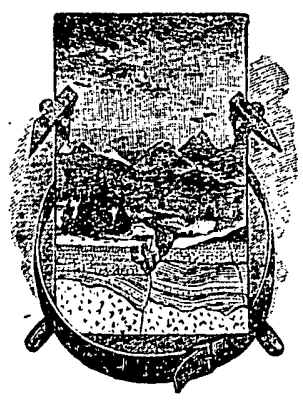

WASHINGTON

GOVERNMENT PRINTING OEFICIE 1900 


\section{CONTENTS.}

Part T. Introduction . . . . . . . . . . .

I. Importance of complete and thorough analyses.............. 13

II. Object and scope of the present treatise: $\ldots \ldots \ldots \ldots \ldots \ldots \ldots \ldots \ldots \ldots \ldots$

III. Statement of analyses. . . . . . . . . . . . . .

IV. Time needed for making an analysis................... 22

V. Two useful aids in chemical manipulation. ................. 23

VI. Limits of allowable error in summation of results. . . . . . . . . . . 24

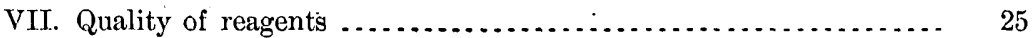

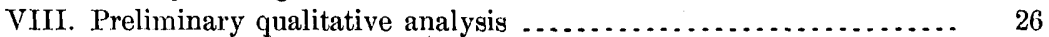

Part II. Methods.............................................. 27

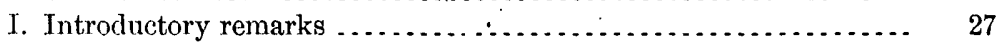

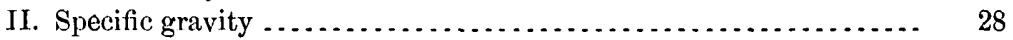

By suspension in water........................ 28

Ordinary method........................... 28

Penfield's method........................... 29

Pycnometer method . ............................ 29

Heavy solutions not suitable for rocks. . . . . . . . . . . . . . $\quad 31$

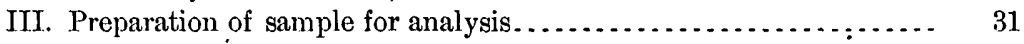

Quantity of rock to be crushed...................... 31

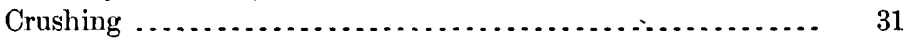

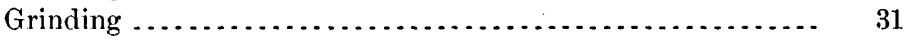

Weight of ground sample........................ 32

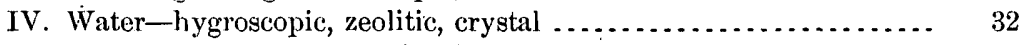

Importance of employing air-dry powder for analysis. . $\quad 32$

Temperature of drying......................... 33

Cautionary hints . ............................ 33

Procedure in special cases....................... 34

Argument in favor of including hygroscopic water in summation. ................................. 35

Separate entry of hygroscopic and combined water... $\quad 36$

Is all true hygroscopic water expelled at $100^{\circ} \ldots \ldots \ldots . .36$

Apparatus for the direct determination of water at different temperatures.................................... 36

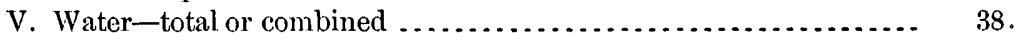

Arguments against "loss on ignition" method ............. 38

Direct. weighing of the water without the use of absorption tubes-

Penfield's methods.................................... 38

For minerals easily deprived of their water. . . . . . . . 38

For minerals not easily deprived of their water...... $\quad 39$

Direct weighing of the water in absorption tubes. . . . . . . . . 40

Penfield's procedure........................ 40

Gooch's apparatus. ........................... 41

Chatard's apparatus....................... 45

Merits of the above three forms of apparatus. . . . . . $\quad 45$

Jannasch's methods ........................... 45 
Part II. Methods-Continued.

VI. Silica, separation from alumina, etc...................... 47

Alternative methods of decomposition.................. 47

Preliminary remarks............................ 47

Decomposition of refractory silicates by hydrochloric acid under pressure

The boric-oxide method .......................... 49

Preparation of the boric oxide.................. 49

Treatment of easily decomposable silicates .......... 49

Treatment of refractory silicates .................. 49

Further treatment after fusion................... $\quad 50$

Possible.objections to the boric oxide method....... 50

The sodium carbonate method..................... $\quad 50$

Purity of the sodium carbonate used as a flux ....... 50

Precautions in fusing ......................... 51

Treatment after fusion........................ 51

Subsequent treatment. ............................. 52

Drying and testing of silica................... 52

Platinum in filtrates.......................... 54

VII. Metals precipitable by hydrogen sulphide................. 54

VIII. Aluminum, total iron.................................... 54

Indirect method for aluminum........................ 54

Precipitation of aluminum, iron, etc................ 55

Precipitation by ammonia .................... 55

Precipitation by the basic acetate method .......... 55

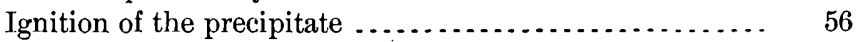

Recovery of silica and possible barium............. $\quad 56$

Estimation of iron in the precipitate of alumina, etc ........ 57

Without regard to the presence of vanadium........ 57

Having regard to the presence of vanadium........ 58

Determination of the true value for ferric iron .......... 59

Methodsaiming at the more or less directestimation of aluminum. $\quad 59$

After first removing iron as sulphide................. $\quad 59$

By.extraction with.a fixed caustic alkali ............ $\quad 59$

Direct precipitation of alumina.................... $\quad 60$

IX. Manganese, nickel, cobalt, copper, zinc .................... 60

Manganese and rinc .................................... 61

Nickel, cobalt, copper ...................................... 61

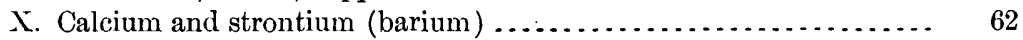

Separation from magnesium........................... 62

Precipitation and ignition of the oxalates together.... 62

Necessity for two precipitations by ammonium oxalate. 62

Separation of strontium (barium). from calcium by ether-alcohol . $\quad 63$

Behavior of barium.................................. 63

Separation of barium from strontium ................... 63

XI. Magnesium ............................................. 64

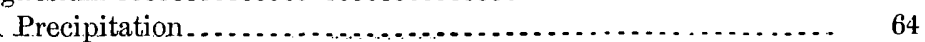

Methods of collecting and igniting the precipitate.......... 66

$\begin{array}{lll}\text { Contamination by and removal of barium and calcium........ } & 67\end{array}$

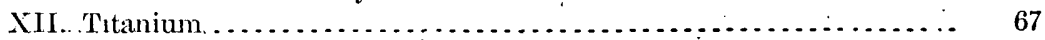

Colorimetric estimation with hydrogen peroxide (Weller's

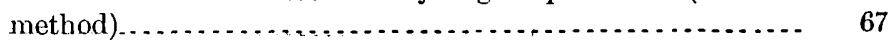

Alternative mode of preparing the test solution......... 69

The colorimetric apparatus and its use ................. $\quad 70$ 
Part II. Methods-Continued.

XII. Titanium-Continued.

Page.

Gooch's gravimetric method :.........................

Gooch's method not directly applicable to rocks con-

taining zirconium .............................

Superiority of the colorimetric and Gooch methods over the older ones ..................................

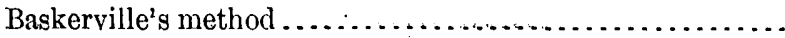

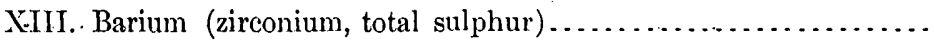

Reasons for estimating barium in a.separate portion of rock powder .............................

Modes of attack and subsequent treatment .........

XIV. Zireonium

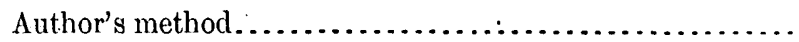

....... Other methods . .................................. 76

XV. Rare earths other than zirconia ............................. 77

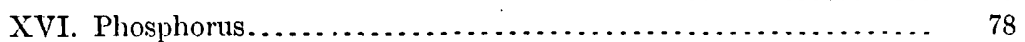

Procedure when material is ample................. 78

Procedure when material is scanty ................. 79

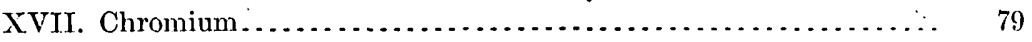

Gravimetric method ............................. 80

Colorimetric method .......................... $\quad 80$

Preparation and strength of staridard solution..... 80

Preparation of the test solution............... 80

Comparison of colors ....................... 81

A few comparative data ......................... 81

XVIII. Vanadium (chromium), molybdenum.................... 82

Distribution of vanadium and molybdenum........... 82

Description of method ........................... 82

Application of the method in presence of relatively much chromium ............................... 85

Condition of vanadium in rocks .................... 88

XIX. Ferrous iron ................................... 88

Comparison of the sealed-tube and hydrofluoric-acid methods; comparative worthlessness of the former in rock analysis..

The modified Mitscherlich method................... 90

Strength of acid.......................... 90

Filling, sealing, and heating of the tube.......... 90 .

Reason for introducing gas and sealing as above directed ................................ 91

The hydrofluoric-acid method ...................... 92

Pratt's modification ......................... 94

Influence of sulphides, vanadium, and carbonaceous matter on the determination of ferrous iron ............... 94 .

Sulphides............................... 94

a Vanadium ............................. 95

Carbonaceous matter ...................... 96

Uncertainties of the ferrous iron determination . . . . . . . . 96

XX. Alkalies .......................................... 96

The Lawrence Smith method ...................... 96

Treatment of the mineral powder.............. 97

Separation of calcium and sulphuric acid........ 98

Precipitation of potassium ................... 98

Lithium ................................... 99

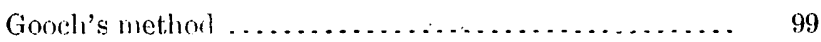


Part II. Methods-Continued.

XX. Alkalies-Continued.

Page.

Separation of alkalies by other methods............. 100

The mercuric-oxide method................ 100

The ammonium-carbonate method............ 100

The amyl-alcohol method.................... 101

XXI. Carbon dioxide, carbon............................. 101

XXII. Chlorine....................................... 103

XXIII. Fluorine....................................... 103

XXIV. Sulphur ........................................ 105

XXV. Boron ........................................ 107

XXVI. Nitrogen....................................... 107

XXVII. Special operations ............................... 108

Detection of nepheline in presence of olivine........... 108

Estimation of soluble silica........................ 109

XXVIII. Estimation of minute traces of certain constituents.......... 110 


\section{L L U S T R A T' I O N S.}

Page.

Fic. 1. Platinum-tipped crucible tongs ............................ 23

2. Radiator for rapid and safe evaporation .................... 24

3. Chatard's form of drying oven for water determinations ........... 37

4. Penfield's tubes for water determination in minerals .............. 39

5. Penfield's fire brick and charcoal oven for use in determining water .. 40

6. Tube for water determination according to Penfield .............. 40

7. Modified form of the Gooch tubulated platinum crucible for the determination of water .............................................. 42

8. Details of Gooch crucible for determining water ................. 43

9. A rrangement during fusion of Gooch apparatus for determining water . 44

10. Glass tube for determination of water (Jannasch) ............... 46

11. Glass tube for determination of water in special cases (Jannasch) .... 46

12. Apparatus for colorimetric determinations .................... 70

13. Cooke's apparatus for the determination of ferrous iron ........... 98

14. J. Lawrence Smith's crucible for alkali determinations............ 97

15. Compact form of apparatus for estimation of carbon dioxide ........ 102 



\title{
LETTER OF TRANSMITTAL.
}

\author{
Departiment of the Interior, \\ United States Geologrcal Surver, \\ - Washington, D. C., Juily 13, 1900.
}

SIR: I herewith submit, with a request for its publication as a bulletin of the United States Geological Survey, a manuscript dealing in very considerable detail with the subject of silicate rock analysis, with special regard to the practice in the Survey laboratory. It represents, in largely rewritten and much extended form, that portion of Bulletin 148 which dealt with the same subject. The present publication seems fully justified by the demand on the part of mineral chemists for Bulletin 148, the supply of which available for sale has been exhausted.

Very respectfully,

W. F. Hrmlibiand,

Chemis:

Hon. Charlis D. Walcott,

Directom United States Geological Survey. 



\title{
SOME PRINCIPLES AND METHODS OF ROCK ANALYSIS.
}

\author{
By W: F. Hillebrand.
}

PART I.-INTIRODUCTION.

\section{IMPORTANCE OF COMPLETE AND THOROUGH ANALYSES.}

The composition of the ultimate ingredients of the earth's crust-the different mineral species which are there found and of many of which its rocks are made up-was the favorite theme of the great workers in chemistry of the earlier half of this century, and for the painstaking care and accuracy of Berzelius, Wöhler, and others the mineralogists and geologists of to-day have need to be thankful. Considering the limited facilities at their disposal in the way of laboratory equipment and quality of reagents, the general excellence of their work is little short of marvelous. As an outgrowth of and closely associated with the analysis of minerals came that of the more or less complex mixtures of them-the rocks - to aid. whose study by the petrographer and geologist a host of chemists have for many decades annually turned out hundreds of analyses of all grades of quality and completeness. With the growth and extraordinary development of the so-called organic chemistry inorganic chemistry gradually fell into a sort of disfavor. In many, even the best, European laboratories, the course in mineral analysis, while maintained as a part of the curriculum of study, became but a subordinate prelude to the ever-expanding study of the carbon compounds, whose rapid multiplication, offering an easy and convenient field for original research and possible profit, proved a more tempting opening to young chemists than the often-workedover and apparently exhausted inorganic pasture. For one student devoting his time to higher research on inorganic lines were perhaps fifty engaged in erecting the present enormous structure of carbon chemistry. The instruction afforded the student in mineral analysis 
was confined to the ordinary separations of the commoner ingredients occurring in appreciable quantities, with little regard to supposed traces and with still less attempt to find out if the tabulated list really comprised all that the mineral or rock contained.

With the introduction of improved methods of examination by the petrographer, especially as applied to thin rock sections, and the use of heavy solutions, whereby, on the one hand, the qualitative mineral composition of a rock could be preliminarily ascertained with considerable certainty, and on the other, chemical examination of the more or less perfectly separated ingredients was rendered possible, a great help and incentive was afforded to the few chemists engaged in rock analysis. The microscope often obviated in part the necessity for tedious and time-wasting qualitative tests, and the heavy solutions, by permitting the concentration and separation of certain components, facilitated the detection of elements whose existence had long been overlooked.

Meanwhile in the progress of chemistry new methods and reagents for qualitative detection, and quantitative separation and estimation were gradually being discovered and devised. The supposed adequacy of some well-established methods was shown to be unwarranted; some had to be discarded altogether; others were still utilizable after modification. In the light thus shed it became possible to explain many hitherto incomprehensible variations in the composition of some rock species or types, as shown in earlier analyses, and in not a few cases it appeared that the failure to report the presence of one or more elements had obscured relations and differences which more thorough examination showed to exist (see pp. 16-17). Consequently there arose a feeling of distrust of much of the older work in the minds of those chemists and petrographers best fitted to judge of its probable qualities. This, and the incompleteness of nearly all the earlier work (and much of that of to-day, unfortunately), as shown by the largely increased list of those elements now known to enter into the normal composition of rocks, is rendering the old material less and less available to meet the increasing demands of the petrographer.

And yet these demands on his part are, with few exceptions, by no means so exacting as they should be. Often the analysis is intrusted to the hands of a student without other experience than that gained by the analysis of two or three artificial salts and as many comparatively simple natural minerals, and with a laboratory instructor as adviser whose experience in rock analysis may be little superior to his own. In other words, one of the most difficult tasks in practical analysis is expected to be solved by a tyro, and his results are complacently accepted and published broadcast without question. Even to those thoroughly familiar with the subject rock analysis is a complex and 
often trying problem. Although long practice may have enabled one to do certain parts of it almost mechanically, one is still from time to time confronted with perplexing questions which "require trained judgment to properly meet and answer, and there is still room for important work in some of the supposedly simplest quantitative determinations. If the results are to have any decided value for purposes of scientific interpretation and comparison, they should be the product of one competent to find his way through the intricacies of an analysis in which from fifteen to twenty-five different components are to be separated and estimated with close approach to accuracy, and this a beginner can not hope to do in the majority of cases. The conscientious chemist should have a live interest in this matter. He should work with a twofold purpose in view-that of lightening the labors of those who come after him by enabling them to use his work with less supplementary examination and of thereby enhancing his own reputation by meriting encomiums on work that has stood the test of time.

The petrographer, again, should seek to have his analyses made as complete as possible, and not, as is so often the case, be content with determinations of silica, alumina, the oxides of iron, lime, magnesia, the alkalies, and water. The latter; it is true, are entirely justifiable at times, and may serve the immediate purpose for which they were intended, but their incompleteness may, on the other hand, not only conceal points fruitful of suggestion to the attentive mind, hut, what is of still greater importance, they may be actually misleading. Enough instances of totally inaccurate conclusions to be drawn from them have fallen under my own observation to fully justify this plea in favor of greater completeness in rock: and mineral analyses made for purely scientific purposes.

The importance of the points indicated in the foregoing paragraph is shown by the difference between the analyses given on the following page. The specimens were taken and analyzed at widely separated times and by different persons, it is true, but they were unquestionably from the same rock mass, in which, however much the relative proportions of the different mineral constituents might vary within certain limits, there can be no reason to doubt the general distribution of all the elements shown by the second analysis. 


\begin{tabular}{|c|c|c|}
\hline & $\begin{array}{l}\text { Earlier } \\
\text { analysis. }\end{array}$ & $\begin{array}{l}\text { Later } \\
\text { analysis. } a\end{array}$ \\
\hline $\mathrm{SiO}_{2} \ldots$ & 54.42 & 53.70 \\
\hline $\mathrm{TiO}_{2}$. & …... & 1.92 \\
\hline $\mathrm{Al}_{2} \mathrm{O}_{3} \ldots \ldots \ldots \ldots$ & 13.37 & 11.16 \\
\hline $\mathrm{Cr}_{2} \mathrm{O}_{3} \ldots \ldots \ldots$ & ........ & .04 \\
\hline $\mathrm{Fe}_{2} \mathrm{O}_{3} \ldots \ldots \ldots$ & b. 61 & 3.10 \\
\hline $\mathrm{FeO} \ldots \ldots$ & b3.52 & 1. 21 \\
\hline $\mathrm{MnO} \ldots \ldots \ldots$ & …. & .04 \\
\hline $\mathrm{CaO} \ldots \ldots \ldots \ldots$ & 4.38 & 3.46 \\
\hline Sro $\ldots \ldots \ldots \ldots$ & ...... & .19 \\
\hline $\mathrm{BaO} \ldots$ & $\ldots . .$. & .62 \\
\hline $\mathrm{MgO} \ldots \ldots \ldots$ & 6.37 & $: 6.44$ \\
\hline $\mathrm{K}_{2} \mathrm{O}$ & 10.73 & 11.16 \\
\hline $\mathrm{Na}_{2} \mathrm{O} \ldots \ldots \ldots$ & 1.60 & 1.67 \\
\hline $\mathrm{Li}_{2} \mathrm{O}$ & Trace. & Trace. \\
\hline $\mathrm{H}_{2} \mathrm{O}$ below $110^{\circ} \mathrm{C} \ldots$. & ....... & .80 \\
\hline $\mathrm{H}_{2} \mathrm{O}$ above $110^{\circ} \mathrm{C} \ldots .$. & $c 2.76$ & 2.61 \\
\hline $\mathrm{CO}_{2}, \ldots \ldots \ldots \ldots \ldots$ & 1.82 & ..... \\
\hline $\mathrm{P}_{2} \mathrm{O}_{5}$ & $\ldots . .$. & 1.75 \\
\hline $\mathrm{SO}_{3} \ldots$ & $\cdots$ & .06 \\
\hline$F \ldots \ldots$ & … & $.44^{\circ}$ \\
\hline Cl...................... & ......... & .03 \\
\hline \multirow{3}{*}{ Less $\mathrm{O}$ for $\mathrm{F} \ldots \ldots$} & 99.58 & 100.40 \\
\hline & & .19 \\
\hline & & 100.21 \\
\hline
\end{tabular}

$a \mathrm{~A}$ still more recent analysis of another of the series of rocks of which this is an example has shown that this "later analysis" is itself probably incomplete and incorrect in part-incomplete because of the probable presence of 0.2 per cent or more of $\mathrm{ZrO}_{2}$, incorrect because of the crror in $\mathrm{Al}_{2} \mathrm{O}_{3}$ resulting from having counted the $\mathrm{ZrO}_{2}$ as $\mathrm{Al}_{2} \mathrm{O}_{3}$, and from the fact that titanium is not fully precipitable in presence of zirconium by Gooch's method (the one employed). This latter error involves both the $\mathrm{TiO}_{2}$ and the $\mathrm{Al}_{2} \mathrm{O}_{3}$. (See pp. 72-73.)

$b$ From the fact that repeated determinations of the iron oxides in this and related rocks from the same region show always a great preponderance of ferric oxide, it is not improbable that the figures given for the two oxides in the first analysis were accidentally transposed.

$c$ In the published analysis it does not appear whether this is total water or, as seems probable, only that remaining above $100^{\circ} \mathrm{C}$.

Another instance of similar kind is given below. Here, again, certain differences are explainable by natural variations in the proportions of the constituent minerals, but it can hardly be doubted that $\mathrm{TiO}_{2}$, $\mathrm{BaO}, \mathrm{SrO}, \mathrm{P}_{2} \mathrm{O}_{5}$, and $\mathrm{SO}_{3}$ were present in both specimens in approximately the same amounts. In the earlier analysis determinations of some supposed unimportant constituents were purposely omitted, or made only qualitatively, with results that can not be otherwise than fatal to a full comprehension of the mineralogical nature of the rock. 


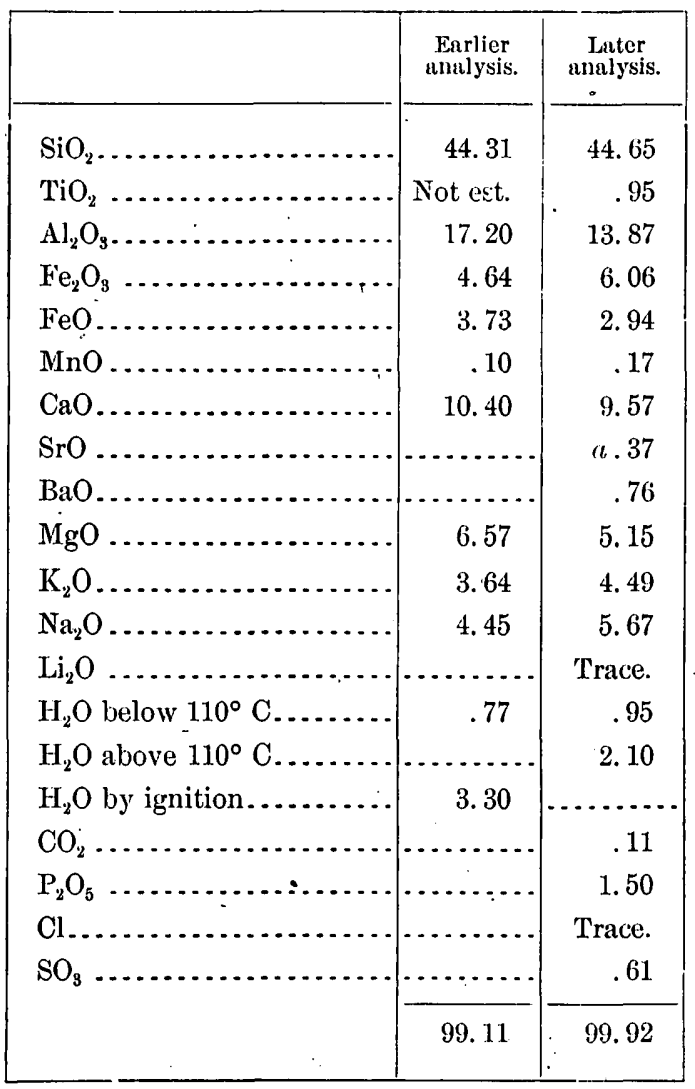

a Not entirely iree from $\mathrm{CaO}$.

Prof. F. W. Clarke has shown that the combined percentages of titanic and phosphoric oxides in rocks of the earth's crust, averaged from hundreds of analyses, is 0.8 per cent. When the determination of these is neglected the error falls upon the alumina. If the latter is then used as a basis for calculating the feldspars, it is easy to see that a very large average error in the latter may result, amounting to several per cent of the rock.

In order to more strongly emphasize the importance of completeness in analysis, a few facts brought out by the hundreds of rock analyses made in this laboratory may be cited. It has been demonstrated most conclusively that barium and strontium are almost neverfailing constituents of the igneous rocks of the United States and of many of their derivatives. These amounts are usually below 0.1 per cent for each of the oxides of those metals, but higher amounts are by no means uncommon. Furthermore, the weight of barium is almost without exception in excess of that of strontium. But a still more important point is that the igneous rocks of the Rocky Mountain region, so far as examined. show far higher average percentages of Bull. $176-2$ 
both metals than the rocks from the eastern and the more western portions of the United States. The following examples serve to illustrate certain types of Rocky Mountain igneous rocks: Of seven rocks forming a Colorado series, six held from 0.13 to 0.18 per cent of $\mathrm{BaO}$, while in the seventh the percentage was 0.43. The $\mathrm{SrO}$ ranged from 0.07 to 0.13 per cent for six, and was 0.28 for that one highest in $\mathrm{BaO}$. Of thirteen geologically related rocks from Montana, embracing basic as well as acid and intermediate types, the range of $\mathrm{BaO}$ was from 0.19 to 0.37 per cent, with an average of 0.30 per cent. Three others of the same series contained 0.10 per cent or less, while the seventeenth carried 0.76 per cent $\mathrm{BaO}$. The $\mathrm{SrO}$ ranged from 0.37 per cent in the last instance to an average of 0.06 for the other sixteen. Certain - peculiar rocks from Wyoming carry from 0.62 to 1.25 per cent $\mathrm{BaO}$, and from 0.02 to 0.33 per cent SrO. Surely this concentration of certain chemical elements in certain geographic zones has a significance which future geologists will be able to interpret, if those of to-day are not.

Again, vanadium is an element which few chemists have ever thought of looking for in igneous rocks, though it has long been known to occur in magnetites and other iron ores. Hayes, in 1875, reported its occurrence in a great variety of rocks and ores. Quoting from Thorpe's Dictionary of Chemistry: "It is said to be diffused with titanium through all primitive granite rocks (Dieulafait), and has been found by Deville in bauxite, rutile, and many other minerals, and by Bechi and others in the ashes of plants and in argillaceous limestones, schists, and sands." It is further reported to comprise, as the pentoxide, up to 0.1 per cent of many. French and Australian clays, $0.02-0.03$ per cent of some basalts, 0.24 per cent of a coal of unknown origin, and 0:45 per cent of one from Peru. Still later examinations in this laboratory of about 100 rocks, chiefly igneous, covering the whole territory of the United States, show not only its general qualitative and quantitative distribution, but that it predominates in the less siliceous igneous rocks and is absent, or nearly so, in those high in silica. In some of the more basic rocks it occurs in sufficient amount to seriously affect the figures for the oxides of iron unless separately estimated and allowed for (see p. 96)--a matter of considerable importance, since the petrographer lays great stress on accuracy in their determinations.

This same investigation has also thrown some light on the distribution of molybdenum, which seems to be confined to the more siliceous rocks and to occur in quantities far below those commonly found for vanadium.

Finally, had it not been the writer's practice of late years to look for sulphur in rocks, even when no sulphides were visible to the eye, its almost invariable presence in the form of sulphide, and consequent 
connection with the long mystifying lack of agreement between results for ferrous iron obtained by the Mitscherlich and the hydrofluoricacid methods, might not have been suspected. (See p. 89.)

While strongly upholding the necessity for more thorough work, necessarily somewhat at the expense of quantity, it is far from the writer's intention to demand that an amount of time altogether disproportionate to the immediate objects to be sought should be expended on every analysis. But it is maintained that in general the constituents which are likely to be present in sufficient amount to admit of determination in the weight of sample usually taken for analysis-say 1 gram for $\mathrm{SiO}_{2}, \mathrm{Al}_{2} \mathrm{O}_{3}$, etc., to 2 grams for certain other constituents-should be sought for, qualitatively at least, in the ordinary course of quantitative work, and their presence or absence noted among the results. If present in little more than traces, that knowledge alone may suffice, for it is often more important to know whether or not an element is present than to be able to say that it is there in amount of exactly 0.02 or 0.06 per cent. In the tabulation of analyses a special note should be made in case of intentional or accidental neglect to look for substances which it is known are likely to be present. Failure to do this may subject the analyst to unfavorable criticism, when at some future time his work is reviewed and the omissions are discovered by new analyses.

Finally, whenever possible, a thorough microscopical examination of the rock in thin section should precede the chemical analysis. This may be of the greatest aid to the chemist in indicating the presence of unusual constituents, or of more than customary amounts of certain constituents, whereby, possibly, necessary modifications in the analytical procedure may be employed without waste of time or labor. ${ }^{1}$

\section{OBJECT AND SCOPE OF THE PRESENT TREATISE.}

The literature relating to analysis of silicates is extensive but scattered, and in no single article is there to be found a satisfactory exposition of the methods to be followed or the precautions to be observed, especially in the search for some of the rarer constituents or those which, without being rare, have been of late years recognized as occurring persistently in small amounts. It is not intended to make this little volume a treatise on mineral analysis, but it is believed that the experience gained by the chemists of this Survey during the twenty years since the establishment of its first chemical laboratory

1'The foregoing tables and accompanying remarks, including several sentences preceding the tables, have been largely taken.from the writer's papers entitled "A plea for greater completeness in chemical rock analysis," published in the Journal of the American Chemical Society, Vol. XVI, pp. 90-93, 1894; also in the Chemical News, Vol. LXIX, p. 163, 1894. See also "Distribution and quantitutive occurrence of vanadium and molybdenum in rocks of the United States," in the American Journal of Science, 4th series, Vol. VI, p. 209, 1898, and Chemical News, Vol. LXXVIII, p. 216, 1898. 
in Denver may pe usefu to most chemists interested in mineral and especially rock analysis.

The original publication of these data in Bulletin No. 148 was primarily intended to show the principles and methods according to which the major part of the very many hundreds of analyses therein brought together had been executed, and thus to furnish a partial measure of the trustworthiness of those analyses, rather than to serve as a practical manual of rock analysis. But the use which has been made by mineral chemists of that bulletin has seemed to render it advisable to amplify somewhat in detail and to add, besides a few new methods, a number of alternative ones which are known or believed to be good, in order that those who may wish to use this treatise as a practical guide shall have a choice from which to select in case the rather expensive apparatus or complicated arrangements sometimes preferred are not available. Where silicate analyses are very frequently made, however, it is a saving of time and of money in the end to set up permanent arrangements for convenience in estimating water, carbon dioxide, ferrous iron, making reductions in hydrogen, etc.

Stress will be laid on those points meriting particular attention, and now and then a brief discussion or criticism of methods elsewhere in vogue may be entered into.

In the earlier years of the existence of the Washington laboratory opportunity was afforded for the testing of novel methods and the devising of new ones, with most excellent results, as shown especially by the methods for separation of titanium, of lithium, and of boron, due to Prof. F. A. Gooch, to whose inventive skill chemists owe likewise the perforated filtering crucible and the tubulated platinum crucible arrangement for the estimation of water. Of late years the press of routine work has been such as to more fully fill up the time of the much-reduced chemical force, and as a consequence it has been found impossible to subject to critical trial several separation methods of recent origin, some of which seem to be full of promise, or to follow out certain lines of investigation which have been suggested by the observations made in this laboratory. This, then, must be offered in explanation if, in the following discussion, it may seem to some that any of the methods followed are too conservative. In general the discussion will be confined strictly to such separations as may be required in the analysis of an igneous, metamorphic, or sedimentary silicate rock of complex mineralogical composition, in which the majority and possibly all of the ingredients in the list given below may occur in weighable or readily discoverable quantities:

$\mathrm{SiO}_{2}, \mathrm{TiO}_{2}, \mathrm{ZrO}_{2}, \mathrm{Al}_{2} \mathrm{O}_{3}, \mathrm{Fe}_{2} \mathrm{O}_{3}, \mathrm{Cr}_{2} \mathrm{O}_{3}, \mathrm{~V}_{2} \mathrm{O}_{3}, \mathrm{FeO}, \mathrm{MnO}, \mathrm{NiO}$, $\mathrm{CoO}, \mathrm{MgO}, \mathrm{CaO}, \mathrm{SrO}, \mathrm{BaO}, \mathrm{ZnO}, \mathrm{CuO}, \mathrm{K}_{2} \mathrm{O}, \mathrm{Na}_{2} \mathrm{O}, \mathrm{Li}_{2} \mathrm{O}, \mathrm{H}_{2} \mathrm{O}$, $\mathrm{P}_{2} \mathrm{O}_{5}, \mathrm{~S},{ }^{1} \mathrm{SO}_{3} \mathrm{C},{ }^{2} \mathrm{CO}_{2}, \mathrm{Fl}, \mathrm{Cl}, \cdot \mathrm{N}$.

The special problems often arising in the analysis of rocks of extra- 
terrestrial origin-the more or less stony meteorites-will not be considered. An analysis of that kind should never be intrusted to the novice, - but only to the chemist who has a knowledge of the composition and properties of the peculiar mineral constituents of those bodies and a judgment fit to cope with the oftentimes difficult problems presented by them.

Thorium, cerium, and other rare earths are seldom encountered in quantities sufficient to warrant the expenditure of the time necessary for their isolation. A search for them qualitatively, even, is at present rarely justifiable unless there is microscopic or other evidence of the presence of minerals likely to contain them. Tantalum, columbium, boron, and glucinum have never been certainly metwith in the writer's experience, and yet they must be present in certain rocks, and doubtless traces have been overlooked at times. There is no reason to suppose that other elements may not be found by careful search, possibly all in tue known category, and, indeed, Sandberger's researches have shown to what an extent this is true of a large number of those elements contributing to the filling of metalliferous veins. But those in the above list may usually be estimated with ease in weights of from one-half to 2 grams.

If the point be raised that many of the published analyses emanating frộm the Survey laboratories, even the earlier ones of the writer, are not in accord with the advocacy of completeness contained in the foregoing pages, it may be remarked that these ideas have been to a considerable degree evolved during a personal experience of twenty years in this line of work, and that frequently the exigencies were such as to compel restriction in the examination. Where the latter has been the case subsequent developments have in some cases shown it to be bad policy in every respect. It is better, both for the geologist and the chemist, to turn out a limited amount of thorough work than a great deal of what may prove to ive of more than doubtful utility in the end.

\section{STATEMENT OF ANALYSES.}

Until recently it has been the practice in this laboratory to tabulate the constituents of a rock somewhat in the order of their determination, beginning with $\mathrm{SiO}_{2}$ as the chief constituent. and grouping together all chemically related oxides, as shown, for instance, on pages 16 and 17 .

From a strictly scientific point of view a chemical classification founded on a separation into basic and acidic atoms or radicals would be more satisfactory, but until we learn to find out what silicic radicals are present and in what relative amounts, also how much free silica there may be, it is useless to think of employing the arrangement so valuable in stating water analyses. 
Of late petrographers have begun to demand, with considerable reason, an arrangement "which shall bring the essential chemical features-both the percentage figures and the molecular ratios-prominently and compactly before the eye, so that the general chemical character and the relations of the various constituents may be seen at a glance." 1

In accordance with this demand it is now our practice to follow pretty closely the arrangement proposed by Pirsson and very recently strongly advocated by Washington (loc. cit.), namely:

$\mathrm{SiO}_{2}, \mathrm{Al}_{2} \mathrm{O}_{3}, \mathrm{Fe}_{2} \mathrm{O}_{3}, \mathrm{FeO}, \mathrm{MgO}, \mathrm{CaO}, \mathrm{Na}_{2} \mathrm{O}, \mathrm{K}_{2} \mathrm{O}, \mathrm{H}_{2} \mathrm{O}$ (above 105-. $110^{\circ}$ C.), $\mathrm{H}_{2} \mathrm{O}$ (below 105-110 C.), $\mathrm{CO}_{2}, \mathrm{TiO}_{2}, \mathrm{ZrO}_{2}, \mathrm{P}_{2} \mathrm{O}_{5}, \mathrm{SO}_{3}, \mathrm{Cl}$, Fl, $\mathrm{S}\left(\mathrm{FeS}_{2}\right), \mathrm{Cr}_{2} \mathrm{O}_{3}, \mathrm{~V}_{2} \mathrm{O}_{3}, \mathrm{NiO}, \mathrm{CoO}, \mathrm{CuO}, \mathrm{MnO}, \mathrm{SrO}, \mathrm{BaO}, \mathrm{Li}_{2} \mathrm{O}, \mathrm{C}$, $\mathrm{NH}_{3}$.

By this arrangement the nine constituents which in the great majority of cases determine the character of the rock are placed at the head of the list, thus greatly facilitating the comparison of different analyses similarly arranged, especially when, as Washington recommends; the molecular ratios are calculated for these leading constituents and placed immediately after the corresponding oxides. The order of the remaining members is determined somewhat by the following considerations: $\mathrm{CO}_{2}$ is placed next after $\mathrm{H}_{2} \mathrm{O}$, since these two are generally a measure of the alteration the rock may have undergone. $\mathrm{TiO}_{2}$ and $\mathrm{ZrO}_{2}$ naturally follow $\mathrm{CO}_{2}$ on chemical grounds, and $\mathrm{SO}_{3}$ and $\mathrm{Cl}$, being common constituents of the sodalite group, are conveniently placed together.

\section{TIME NEEDED FOR MAKING AN ANALYSIS.}

The question has often been put, "How long does it take to complete an analysis of this kind?" This will depend, of course, on the mineral complexity of the sample and on the personal factor of the individual worker. If there is a competent assistant to do the grinding, and specific-gravity determinations are not required, it is quite possible after long experience for a quick worker to learn to so economize every moment of time in a working day of seven hours, with an abundance of platinum utensils and continuous use of air and water baths through the night, as to finish every three days, after the completion of the first analysis, barring accidents and delays, one of a series of rocks of generally similar character, each containing from eighteen to twenty quantitatively determinable constituents, excluding, for instance, fluorine, carbon as such, nitrogen, metals of the hydrogen sulphide group, and cobalt. On one occasion a series of fourteen rocks, of comparatively simple composition, was completed in one month, with the help of an assistant who made the phosphorus

'H. S. Washington, The statement of rock analyses: Am. Jour. Sci., 4th series, Vol. X, p. 61, 1900. 
and ferrous iron determinations. But such an output of work is more than exceptional and implies an unusual freedom from those occasional setbacks to which every chemist is exposed.

It should here be remarked that the Survey laboratory is most exceptionally well supplied with all kinds of platinum vessels and utensils, so that it is rare indeed for delay to arise through lack of dishes of even the largest sizes.

\section{TWO USEFUL AIDS IN CHEMICAL MANIPU- LATION.}

In connection with the foregoing remarks it is in place to mention two aids to the chemist which are in constant lise in this laboratory and have come to be well-nigh indispensable. Neither is novel in principle and both arc in use elsewhere, but they are not so commonly known as they deserve to be, hence this allusion to them.

Fig. 1 represents a form of platinum-tipped crucible tongs devised by Dr. A. A. Blair many years ago. With them a crucible can be securely grasped and brought into any desired position while still hot. To the contents, if in fusion over the blast flame, can be imparted the rotatory motion so often desirable. Above all, the cover need not be in the slightest degree displaced, as when using the common form of platinum-tipped tongs.

Fig. 2 represents a very useful adjunct to the worktable and especially to the draught cupboard, whereby the liquid contents of crucibles can be speedily evaporated at almost any desired temperature and the dehydration of many solids effected much more safely than on an iron plate or sand bath. I do not recall who originated this form of air bath, but it has been in use here for over fifteen years and is identical in principle with the later Nickel-becher of Jannasch. Nickel undoubtedly has a certain advantage in not rusting as does iron, but the form depicted in $R$ of fig. 2 can easily be made anywhere of sheet iron riveted at the joint, the bottom (not shown in the figure) being securely

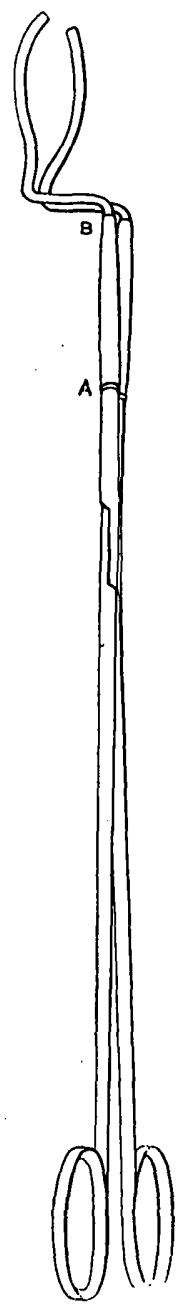

Fra. 1.-Platinum-tipped crucible tongs. The parts $A B$, also of heavy platinum, are hollow, to serve as sockets for the cheaper metal of the handles.

held by a flange at the extremity of the truncated cone. A crucible placed on the platinum triangle becomes uniformly heated by hot air, and large quantities of liquid, cven sulphuric acid, can be thus volatilized in a short time without ebullition or spattering. 
VI. LIMITS OF ALLOWABLE ERROR IN SUMMATION OF ANALYTICAL RESULTS.

As is well known, a complete silicate rock analysis which foots up less than 100 per cent is generally less satisfactory than one'which shows a summation somewhat in excess of 100 . This is due to several

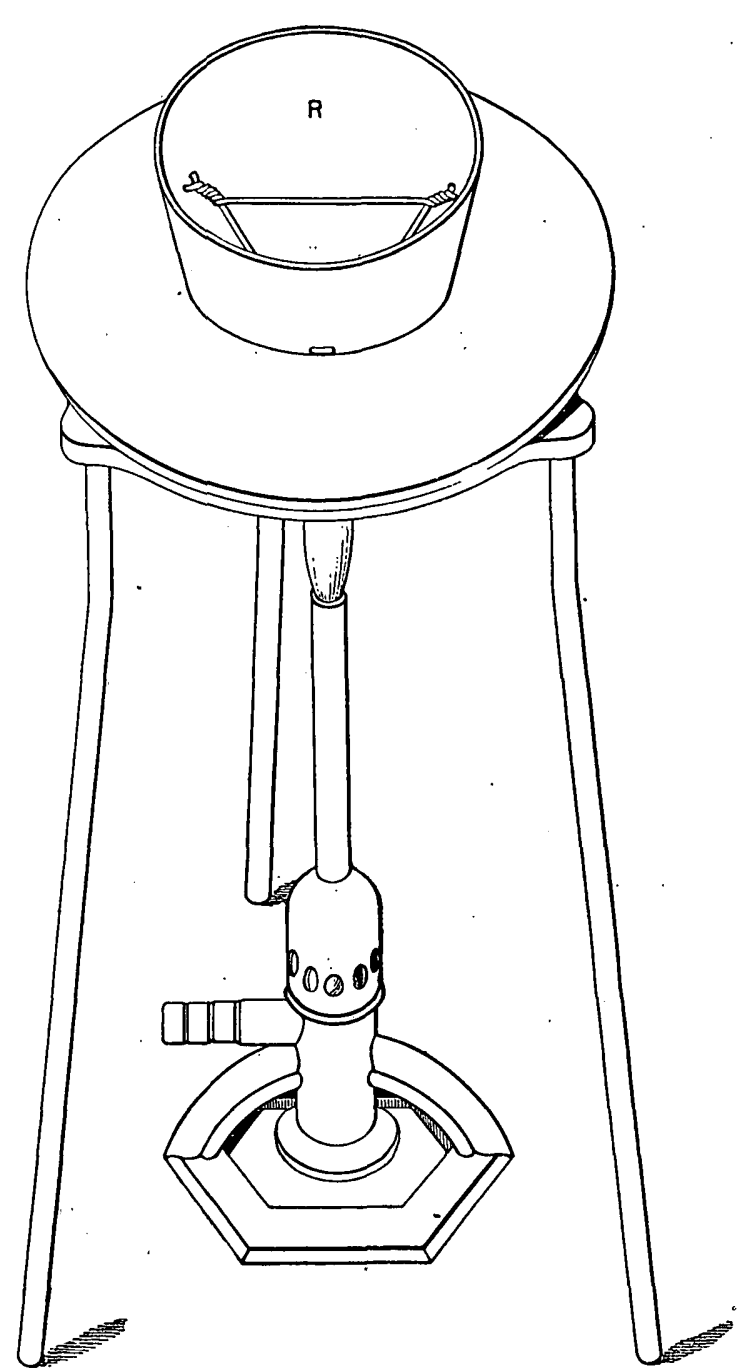

Fig. 2.-Radiator for rapid and safe evaporation. $R$ is of sheet iron, also nickel (Jannasch). Various sizes. A convenient height is $7 \mathrm{~cm}$, width at top $7 \mathrm{~cm}$ and at bottom $5 \mathrm{~cm}$. causes. Nearly all reagents, however carefully purified, still contain, or extract from the vessels used, traces of impurities, which are eventually weighed in part with the constituents of the rock. The dust entering an analysis from first to last is very considerable, washings of precipitates may be incomplete, and if large filters are used for small precipitates the former may easily be insufficiently washed.

Given the purest obtainable reagents, an ample supply of platinum, facilities for working, and a reasonably clean laboratory, there is no excuse for failure on the part of a competent chemist to reach a summation within the limits 99.75 and 100.50. Failure to attain 100 per cent in several of a series of analyses of similar nature should be the strongest evidence that something has been overlooked. Excess above 100.5 per cent should be good ground for repeating portions of the analysis in order to ascertain where the error lies, for it is not proper to assume that the excess is distributed over all determined constit- 
uents. It is quite as likely, in fact more than likely, to affect a single determination and one which may be of importance in a critical study of the rock from the petrographic side.

\section{QUALITY OF REAGENTS.}

It is due to say that all analyses performed in the Survey laboratories have been made with the purest reagents obtainable, either by purchase in the open market or by special preparation on the part of manufacturers or in the laboratory. The best acids made in this country are of a high grade and need no redistillation except for special experiments. Ammonia has always been redistilled at short intervals; and no sodium carbonate which exceeds $2 \frac{1}{2}$ milligrams of total impurity (see p. 50) in 20 grams ( 0.012 per cent) is used for the main portions, in which silica, alumina, etc., are to be estimated. For other portions, as phosphoric acid, fluorine, sulphur, a poorer grade is entirely allowable, provided it is free from the element to be determined, and from any other which might interfere with its estimation.

Hydrofluoric acid was always freshly distilled with potassium permanganate until the introduction of ceresine botties afforded an article sufficiently pure for all but the most exacting work. Care must be exercised even yet, however, that no particles of paraffin or ceresine are floating on the acid, and that the latter is free from traces of chlorine whenever it is to be used for attacking silicates with a view to estimating chlorine (p. 103).

Potassium bisulphate has usually been prepared in the laboratory from sulphuric acid and potassium sulphate, since it is not always to be bought of satisfactory quality. Even then the normal sulphate had first to be examined, for it has been found to contain, on different occasions, notable amounts of lead, calcium, and silica.

The phosphoruis salt used for precipitating magnesium has been found to contain iron, and calcium is almost always a constituent of ammonium oxalate. The latter has therefore to be purified or specially prepared, as also oxalic acid, ammonium chloride (in which latter: manganese has been observed), and occasionally other reagents. Some hydrogen peroxide contains fluorine, which renders it unfit for use as a chemical reagent.

A "C. P." label is no guaranty whatever of the purity of a reagent; hence no chemicals should be taken on trust because of bearing" such a label. Every new purchase should be examined, if it is one in which purity is a desideratum. In general all so-called "C. P." chemicals should at least stand the tests laid down by Krauch."

Of late years the appearance upon the market of so-called guaranteed reagents promised to meet a long-felt want. But experience has 
shown that with the pioneer in this line at least the guaranty amounts to nothing, the reagents being sometimes worse than the "C. P." articles emanating from sources which make no claim to special purity for their goods, and redress being unobtainable. The "guaranteed reagent" needs checking as much as any other.

VIII. PRELIMINARY QUALITATIVE ANALYSIS.

A complete qualitative analysis of a rock, preceding the quantitative examination, is in most cases a sheer waste of time. A few constituents may now and then be specially looked for, but in general time is saved by assuming the presence of most of them and proceeding on that assumption in the quantitative analysis. 
PART II. METHODS.

\section{INTRODUCTORY REMARKS.}

The order hereinafter followed in describing the various chemical separations has little relation to the affinities of the constituents of the rock, but those are grouped together which can be conveniently determined in the same portion of rock powder. Thus, in the main portion are usually determined $\mathrm{SiO}_{2}, \mathrm{TiO}_{2}, \mathrm{MnO}, \mathrm{NiO}, \mathrm{CaO}, \mathrm{SrO}$, $\mathrm{MgO}$, total iron, and the combined weight of all the following: $\mathrm{\Lambda I}_{2} \mathrm{O}_{3}$, $\mathrm{TiO}_{2}, \mathrm{P}_{2} \mathrm{O}_{5}, \mathrm{ZrO}_{2}$, all iron as $\mathrm{Fe}_{2} \mathrm{O}_{3}$, and nearly if not quite all vanadium as $\mathrm{V}_{2} \mathrm{O}_{5}$, also perhaps rare earths if present. In a separate portion is estimated $\mathrm{FeO}$, and also the total iron, as well as $\mathrm{BaO}$, if these last are desired as checks. The alkalies need a portion for themselves. In another, $\mathrm{ZrO}_{2}, \mathrm{BaO}$, and total sulphur are very conveniently determined. For $\mathrm{V}_{2} \mathrm{O}_{3}$ and $\mathrm{Cr}_{2} \mathrm{O}_{3}$ still another and usually much larger portion is to be used. Determinations of $\mathrm{CO}_{2}, \mathrm{C}, \mathrm{H}_{2} \mathrm{O}, \mathrm{Fl}, \mathrm{Cl}$, are all best made in separate portions of substance, though various combinations are possible, as $\mathrm{CO}_{2}$ and $\mathrm{H}_{2} \mathrm{O}, \mathrm{C}$ and $\mathrm{H}_{2} \mathrm{O}$, or $\mathrm{H}_{2} \mathrm{O}, \mathrm{Fl}$, and $\mathrm{Cl}$. In fact, by a judicious selection and combination of methods a very satisfactory analysis can sometimes be made on 4 grams of material without omission of anything of importance, though the time consumed will be greater than if ample material is available.

As an illustration of the advantage to be gained by a little judgment in the combination of methods, the case of sulphur, barium, and zirconium may serve. Many chemists never look for the second and third of these, but by following the procedure given on pages $74-76$ very little more labor is expended in confirming their presence or absence than that of sulphur alone.

With only occasional exceptions, nearly all the constituents mentioned on page 20 can be estimated if present in portions of powder not exceeding 1 gram each in weight.

This is a convenient weight to. take for the main portion in which silica, alumina, etc., the alkaline earths, and magnesia are to be sought; but it should, in general, be a maximum, because if larger the precipitate of alumina, etc., is apt to be unwieldy. Its weight can not often be much reduced with safety if satisfactory determinations of manganese, nickel, and strontium are to be expected. For the alkali por- 
tion one-half gram is a very convenient weight. In general, it may be made a rule not to use more than 2 grams for any portion which has to be fused with an alkali carbonate, as for sulphur, fluorine, and chlorine. For carhon dioxide the weight may rise to 5 grams, or even more, if the amount of this constituent is very small, without expenditure of any more time than is required by 1 gram, and with correspondingly greater approach to correctness in the result. For vanadium also a larger weight than 2 grams is usually demanded.

\section{SPECIFIC GRAVITY.}

\section{BY SUSPENSION IN WATER.}

Ordinary method.-This determination, when required, is best made upon one or several fragments weighing up to 20 grams. They are held together by a fine platinum wire ready for suspension from the balance, and thus held are placed-in a small beaker to soak over night in distilled water under the exhausted receiver of an air pump, side by side with a similar beaker of water. Boiling is, of course, a much less effective means of removing air than the air pump, and the boiling - water may exert an undesirable solvent and abrading effect. In the morning the wire is attached to the balance arm, the rock fragments remaining immersed in the water; a thermometer is placed in the companion beaker of water, now likewise in the balance case, and the weight is at once taken. Both vessels of water having precisely the same temperature, it is quite unnecessary to wait for the water to assume that of the balance should it not already possess.it. The fragments are now lifted out, without touching the vessel, and carefully transferred to a tared crucible or dish; the wire is removed and at once reweighed, with the precaution that it dips just as far into the water now as when weighted. Hereby a special weighing of the wire out of water is avoided. The sample may'now be dried on the water bath and then at $110^{\circ} \mathrm{C}$. for some hours to certainly expel all absorbed water, and weighed after prolonged cooling in the desiccator. It is better to ascertain the weight of the dry rock after soaking in water than before, in order to avoid the error due to possible breaking off of a few grains between the two weighings. Should the density of the rock in air-dry condition be required, it may be left exposed to the air for a long period after drying and before weighing; ${ }^{1}$ but the difference will only

\footnotetext{
${ }^{1}$ In view of the uncertainty as to what constitutes hygroscopic water (see p. 35), this course is perhaps more to be commended than the former, and seems imperative for certain zeolitic rocks. In such cases it is best to weigh the fragments before putting to soak, and afterwards to collect on a Gooch crucible the grains which may have fallen off in the water. Should no crucible of this kind be available, a paper filter may unhesitatingly be used and incinerated with the powder, owing to the small amount of which the error due to loss of even all its water during ignition is quite negligible.
} 
in exceptional cases affect the second decimal by more than a single unit. For instance, an undried rock of 2.775 specific grevity containing in the uncrushed state the high percentage of 0.3 hygroscopic moisture will have a density of 2.79 when dry; a rock of 2.982 specific gravity, undried, will have a density of 3.00 after removal of 0.3 per cent of moisture. The difference becomes greater as the density of the rock increases.

This method of ascertaining the specific gravity of rocks is certainly more convenient than, and for compact rocks is believed to be decidedly.preferable to, that of the pycnometer, in which the fragments must be reduced to small size with consequent formation of more or less powder, which is subject to slight loss in the various manipulations. To exclude this powder and employ only small frag ments would introduce a possible source of error, since it is likely to consist largely of the most easily abraded minerals and consequently not to have the average composition of the mass. By following the instructions'given above, loss of material is absolutely avoided, a decided saving in time is effected, and considerable weights can be easily employed with consequent lower probable error in the results. To vesicular rocks, however, notably certain lavas, the above pròcedure is, of course, inapplicable, unless the datum is desired for certain considerations in which the relative density of large rock masses as they occur in nature is sought, as for the comparison of building stones or the calculation of large known or assumed areas of particular rocks.

Penfield's method for mineral fragments. - Penfield ${ }^{1}$ recommends the following modification of the suspension method as more convenient than that by the pycnometer in many cases for small fragments of minerals.

After boiling in water, the substance is transferred with water to a smitll glass tube about $8 \mathrm{~mm}$. by $35 \mathrm{~mm}$, provided with a fine platinum wire for suspension. This is weighed full of water in another vessel of water, and again after the removal of the mineral, the weight of which is found after drying.

This method is, of course, more applicable to homogeneous minerals than to rock fragments, and will therefore be applied in rock analysis chiefly to the determination of the specific gravity of the mineral grains separated by heavy solutions or acids.

\section{PYCNOMETER METHOD.}

If the pycnometer has to be used, as is generally the case when the density of any one of the mineral ingredients of a rock is desired after 
separation by one of the approved methods, it being then in a more or less finely divided state, the most accurate procedure is that adopted in this laboratory by Mr. L. G. Eakins a number of years ago. The pycnometer used is one with a capillary stopper, provided with a millimeter scale etched in the glass, the divisions being numbered both ways from the center and calibrated by mercury so that the value of each one in weight of water is known. The capacity of the flask filled with water to the zero division is then calculated for every half degree of temperature from $0^{\circ}$ C. to $30^{\circ}$ C., by making a series of careful weighings, in which, the capacity of the stem being known, it is quite immaterial at what level the water stands provided it is within the limits of the scale. The exact temperature is obtained by an accurate thermometer placed in a companion vessel of similar shape to the pycnometer and containing a like amount of water, both being left in the balance case till its temperature has been nearly or quite assumed, as shown by a second thermometer. The weighing must of course be made before the thread of water has sunk beneath the lowest division, which it will do after a time, even though at first filling the bore to the top of the stopper; and the corrected weight full of water to the zero mark is found by adding or subtracting the needed amount, as shown by the height of the thread on the scale.

For each pycnometer in use, and these are of different sizes, is prepared a table showing its weight, the value of each scale division in grams of water, and the capacity of the flask at different temperatures, as indicated above. The preparation of such a series of flasks is time saved in the end, for the weighing of the flask full of water each time a density determination is made is rendered superfluous. All that is necessary is to look up in the table the weight corresponding to the temperature.

The density of the previously weighed substance in this case is now determined in much the same way, after the unstoppered pycnometer containing it and nearly filled with water has stood with its companion vessiel of water under the air pump the necessary length of time. The water needed to fill the flask is taken from its companion.

All who have used the pycnometer method for fine substances know the difficulty experienced in preventing a portion from being held at the surface, despite all attempts at making it sink. Hence it often happens that a very small portion runs out around the sides of the stopper on inserting it. If the flask rests in a small tared dish the grains thus forced out may be washed down into it and weighed after evaporation in order to get the correct weight of that in the flask; or, after weighing, the contents of the flask may be emptied into a tared dish and the water slowly evaporated off in order to get the weight of the mineral. Usually this way is less to be recommended than the other. 
HEAVY SOLUTIONS NOT SUITABLE FOR ROCKS.

Because of their roughness, porosity, and complex mineral composition the density of rock fragments can not be accurately detcrmined by that of heavy solutions in which they may remain suspended.

\section{PREPARATION OF SAMPLE FOR ANALYSIS.}

\section{QUANTITY OF ROCK TO BE CRUSHED.}

In the great majority of cases a few chips from a hand specimen will well represent the average of the mass, but with rocks in which a porphyritic structure is strongly developed the case is different. Here a large sample should be provided, gauged according to the size of the crystals, and the whole of this should be crushed and quartered down for the final sample. Unless this is done, it is manifest that the analysis may represent any thing but the true average composition of the rock.

\section{CRUSHING.}

Mechanical appliances for reducing samples to fine powder are much in use in technical laboratories, where they answer their purpose more or less satisfactorily, and something similar is needed in those scientific laboratories where rock analysis is of daily occurrence and many samples must be reduced to fine powder in a short space of time. For accurate analyses the use of steel crushers and mortars is out of the question, because of the danger of contamination by particles of metal and the impossibility of cleansing the roughened surfaces after they have been in use a short time. Extraction by the aid of a magnet of steel particles thus introduced into the powder is quite inadmissible, since the rocks themselves, almost without exception, contain magnetic minerals. The method of rough crushing on a small scale found to be most satisfactory in practice is to place each fragment as received on a hard steel plate about $4 \frac{1}{2} \mathrm{~cm}$. thick and $10 \mathrm{~cm}$. square, on which is likewise placed a steel ring $2 \mathrm{~cm}$. high and of about $6 \mathrm{~cm}$. inner diameter, to prevent undue flying of fragments when broken by a hardened hammer. In this way a considerable sample can soon be sufficiently reduced for transfer to the agate grinding mortar with a minimum of metallic contamination.

For breaking large pieces of rock to small sizes a thick iron plate with specially hardened surface and a similarly hardened pounder, such as street pavers use, will probably render the best service, but the hardening must be done with extreme care.

GRINDING.

Of the various grinding arrangements on the market purporting to fulfill their purpose few, if any, observed have met the conditions 
required by the work in hand. Either the mechanical arrangement is complicated or cumbersome, requiring more power or space than is usually at disposal or causing too much noise, or thorough cleansing is difficult and troublesome, or there is likelihood of contamination from oil or grease or lack of facility for the removal of all powder from the mortar. These last defects are especially prominent in those forms in which the mortar is fixed in its setting.

All rock samples have therefore been reduced to powder by hand, involving a great expenditure of time and labor. Ordinarily an extremely fine state of division is unnecessary, except in the case of those portions in which alkalies and ferrous iron are to be estimated or where soluble constituents are to be removed by acids, etc., and in such cases the final grinding can be done at the balance table on a small portion slightly in excess of the quantity to be weighed off.

The process of sifting through fine cloth, the German "Beuteln," is not one always to be commended, because of the time required and, more especially, because of the certainty of contamination by cloth fiber, which in the ferrous-iron portion might affect the result. . Still less should metal sieves be used.

\section{WEIGHT OF GROUND SAMPLE.}

The sample when ground should weigh not less than 10 grams, and preferably 20 in case it should be necessary to repeat or advisable to employ unusually large portions for certain determinations, notably carbonic acid. Rock analysis has in this respect an advantage.over mineral analysis, since material is almost always available in ample quantity and any desired number of separate portions may be used, whereas with a mineral the analyst is frequently compelled to determine many or all constituents in a single, often very small, portion of the powder. This course often involves delay and the employment of more complicated methods of separation than are usually necessary in rock analysis.

\section{WATER-HYGROSCOPIC, ZEOLITIC, CRYSTAL.}

Importance of employing air-dry powder for analysis.-The timehonored custom of drying a powdered specimen before bottling and weighing has long seemed to the writer one that has no sound basis in reason. Its object is of course plain, namely, that of securing a uniform hygroscopic condition as a basis for convenient comparison of analytical results, since some rocks contain more hygroscopic moisture than others. Nothing, however, is more certain than that by the time the substance is weighed it has reabsorbed a certain amount of moisture, small, indeed, in most cases, but very appreciable in others; and 
further, with every opening of the tube moisture-laden air enters and is inclosed with the remainder of the dry powder. It therefore may very well happen that a powder at first dry will, after several openings of the tube, especially at considerable intervals, be nearly as moist as when first inclosed.

It is preferable to weigh the air-dry powder and to make a special. determination of moisture. If all the portions necessary for an analysis are weighed out one after another, or even at different times on the same day, the error due to difference of hygroscopicity in dry and moist weather, which for most of the separate portions is an entirely negligible quantity, is eliminated. Only in the main portion, in which silica and the majority of the bases are to be estimated, can it ever be an appreciable factor.

Temperature of drying.-As to the temperature to be adopted for drying in order to determine so-called hygroscopic moisture, the practice has varied at different times and with different workers, ranging from $100^{\circ}$ to $110^{\circ} \mathrm{C}$. For the great majority of rock specimens it is quite immaterial which of these temperatures is adopted, since no greater loss is experienced at the higher than at the lower temperature, given a sufficient time for the latter. It is the present practice in this laboratory to employ a toluene bath giving a temperature of about $105^{\circ} \mathrm{C}$. Should the results show a very unusually high loss, the powder is reheated at, say, $125^{\circ}$, in order to learn if the loss is progressive with increased temperature. In the affirmative case it may be well to repeat the drying at $100^{\circ}$, for a portion of the loss at $105^{\circ}$ was probably due to combined water from a mineral or minerals in the rock; but in that case even the loss at $100^{\circ}$ may sometimes very well include combined water, in which case drying over sulphuric acid alone may be desirable, or over dry sand.

Cautionary hints. - In this latter connection it is proper to point out certain pitfalls in the path of the unwary, which, however, are far more likely to be encountered in the analysis of minerals, where their influence, may be of far-reaching consequence.

A mineral which loses a great deal of water over sulphuric acid -2 or 3 per cent, for instance-may need an exposure of several days or even weeks for its complete extraction. If the weighings are made from day to day, the apparent limit may be reached long before all water really removable has been taken up by the acid. Whenever the crucible, after weighing, is replaced in the desiccator it is no longer in a dry but a more or less moist atmosphere, and its contents, even when covered, sometimes absorb a part of this moisture and retain it so persistently that the acid is unable to bring the powder beyond its previous state of dryness in the next twenty-four hours. In fact, it may be unable even to reach it unless greater time is allowed. An experiment Bull. 176-3 
on 1 gram of tyrolite, made and published some years ago, seems to illustrate this point in part:

\begin{tabular}{|c|c||r|r|}
\hline $\begin{array}{c}\text { Hours } \\
\text { exposed. }\end{array}$ & \multicolumn{1}{c|}{ Loss. } & $\begin{array}{r}\text { Hours } \\
\text { exposed. }\end{array}$ & Loss. \\
\hline & $\begin{array}{c}\text { Gram. } \\
18\end{array}$ & & Gram. \\
26 & .0231 & 24 & 0.0002 \\
23 & .0083 & 24 & .0003 \\
24 & .0029 & 48 & .0006 \\
23 & .0012 & 24 & .0002 \\
24 & .0001 & 283 & .0380 \\
25 & .0003 & & \\
\hline
\end{tabular}

The experiment might reasonably have been considerea ended after the one hundred and fifty-eighth hour, when a loss of but 0.1 milligram was shown during twenty-four hours; but nevertheless a nearly steady loss of 0.3 milligram per day took place for six days more, and might have been longer observed but for the interruption of the experiment.

Again, it is a common practice to determine the water given off by hydrous minerals in an air bath at temperatures far above $100^{\circ} \mathrm{C}$. To insure accuracy this experiment should not be made in crucibles or dishes which must be cooled in a desiccator. One instance will suffice: A gram of a mineral mixture containing about 17 per cent of water, of which about 3 per cent was driven off at $100^{\circ}$ and 8 or 9 per cent at $280^{\circ}$, was, after several hours' heating at the latter temperature, placed in a desiccator over sulphuric acid and weighed as soon as cold, then replaced and again weighed the next day. It had regained $1 \frac{1}{2}$ per cent of its original weight, although the desiccator was tightly closed and the crucible covered, showing apparently a drying power superior to that of the acid.

A specimen of tyrolite was found on one occasion to lose 10.34 per cent at $280^{\circ}$ C., and on another occasion 14.33 per cent. In the latter case the drying and heating at progressive temperatures had continued during a period of 528 hours, the weighings being made usually from day to day; whereas in the former the duration of the experiment was much shorter and the intervals between weighings were but a few hours each.

Procedure in special cases.-For experiments of the kind just indicated the powder should be heated in a weighed tube, through which a current of dry air can be passed, and allowed to cool therein, or else the water given off should be collected and directly weighed in suitable absorption tubes, even though the long time often required is an objection to this lattter method, since the absorption tube may 
gain weight, other than that of the water from the mineral, sufficient to introduce an appreciable error.

The recent important research of Friedel ${ }^{1}$ well shows what errors are possible in the determination of this easily removable water, since he found that certain zeolites which had been largely dehydrated but not heated to the point of rupture of the molecular net, could then absorb, instead of water, various dry gases in which they might be placed, as carbon dioxide, ammonia, carbon disulphide, and others, even air in large quantities, and certain liquids. In the light of this observation the cause of the great increase of $1 \frac{1}{2}$ per cent in weight of the partially dehydrated mineral mentioned on p. 34 may very possibly be attributed to air from the desiccator instead of moisture, as was at the time supposed. At any rate, as Friedel says, the danger of accepting a loss in weight as an index of the amount of water lost is clearly shown, and thus that method of determining water is for many cases fully discredited. Just what method to adopt must be largely left to the judgment of the operator, who will often be guided by the mineral composition of the rock as revealed by the unaided eye or the microscope.

Friedel (loc. cit.) indicates a means for determining the true weight of water lost by minerals behaving like the zeolites, even without collecting the water lost, namely, by driving out of the dehydrated and weighed mineral, under proper precautions, any air it may have absorbed in the process of drying and cooling, and collecting and measuring this air and thus finding its weight, which, added to the apparent loss, gives the true contents in water.

Argument in favor of inchiding hygroscopic water in summation.The question has been asked: "If the so-called hygroscopic water is not always such, but not infrequently includes combined water, why is not its determination and separate entry in the analysis entirely unnecessary? Why make a distinction, which, after all, may not be a true one?" The question involves the further consideration of the advisability of including in the analysis at all the loss at $100^{\circ}$ or $110^{\circ} \mathrm{C}$. Many petrographers desire to have all analyses referred to a moisturefree basis, in order that they shall be strictly comparable, and therefore would omit the "hygroscopic" water from the list of constituents. This would be eminently proper were it always possible to be sure that the loss at $100^{\circ}$ truly represents mechanically held water. Since it very often represents more, and the determination as to whether or not it does in each case is not always possible, and would add to the time required for the analysis, it seems necessary to include this water. What errors may arise from its exclusion the following rather extreme case well illustrates: Certain rocks of Wyoming in powder form lost from 1 to 2 per cent of moisture at $110^{\circ}$. That not even an appreciable fraction of this was truly hygroscopic the fact of

1 Bull. Soc. Min., Vol. XIX, pp. 14, 94, 1896; Comptes Rendus, Vol. CXXII, p. 1006, 1896. 
the uncrushed rocks losing the same amount fully demonstrates; yet the rule followed by many chemists and petrographers would have involved the removal of all this water as a preliminary to beginning the analysis, and not only would a most important characteristic have passed unnoticed, but the analyst would have reported an incorrect analysis, inviting to false conclusions and possibly serious confusion.

Separate entry of hygroscopic and combined water.-To revert now to the primary question, it may be said that the estimation of the loss at $100^{\circ}$ or $110^{\circ} \mathrm{C}$. and its separate entry in the analysis is advisable as not infrequently affording at once to the lithologist an indication of the mineral character of one or more of the rock constituents, thus perhaps confirming the microscopical evidence or suggesting further examination in that line. An unusually high loss at $100^{\circ}$ would be regarded as probable evidence of the presence of zeolites or other minerals carrying loosely combined water. It has been objected that the true hygroscopic moisture varies with the degree of comminution of the sample and with the condition of the air at the time of weighing, and that it is therefore improper to incorporate it in the analysis; but this variation is ordinarily not at all great. Perhaps the time may come when it will be the rule to ascertain by additional heating at a higher: temperature whether the water lost at $100^{\circ}$ is to be regarded as purely hygroscopic. In such case it would be proper to omit it, and a distinct advance would undoubtedly be scored.

Is all true hygroscopic water expelled at 100\%? - It has been tacitly assumed in the foregoing that true hygroscopic water can all be expelled at $100^{\circ}$, which perhaps is not to be accepted as universally true. Eminent authority holds that it is impossible, in the cases of certain foliaceous minerals, notably the micas, to thus entirely remove it, but that a part is only driven off at higher temperatures. If this is true a further uncertainty is introduced in its determination, which not only strengthens the argument in favor of entering all water in the tabulation, but also serves to emphasize the difficulties of the situation.

APPARA'US FOR THE DIRECT DETERMINATION OF WATER AT DIFFERENT TEMPERATURES.

A form of drying oven devised by Dr. T. M. Chatard ${ }^{1}$ is in use in this laboratory for determining water at different temperatures up to $350^{\circ} \mathrm{C}$, and gives entire satisfaction. It is an asbestos-covered copper box $\mathrm{B}$, shown in different aspects and parts in the accompanying fig. 3. The box is so constructed that the tube with its contents can be removed without detaching from either the drying or collecting tubes, which is a great advantage if it is desired to afterwards apply the direct

${ }^{1}$ Am. Chem. Jour., Vol. XIII, p. 110, 1891; Bull. U: S. Geol. Survey No. 78, p. 84. 
heat of a lamp in order to expel the water retained at $300^{\circ}$ to $350^{\circ} \mathrm{C}$. To facilitate this removal the stand is on rollers, so that after clamping the projecting end of the tube and removing the front of the box $\mathrm{F}$ and the little side pieces $\mathrm{S}$ closing the horizontal slits, the oven can be rolled bodily backward, leaving the tube and its attachments in their original position, ready for further heating over a burner or blast. The removable front $\mathrm{F}$ of the oven is made of two pieces of sheet asbestos board stiffened by an interlaid piece of sheet copper. The inner viece of asbestos board fits snugly into the box, while the

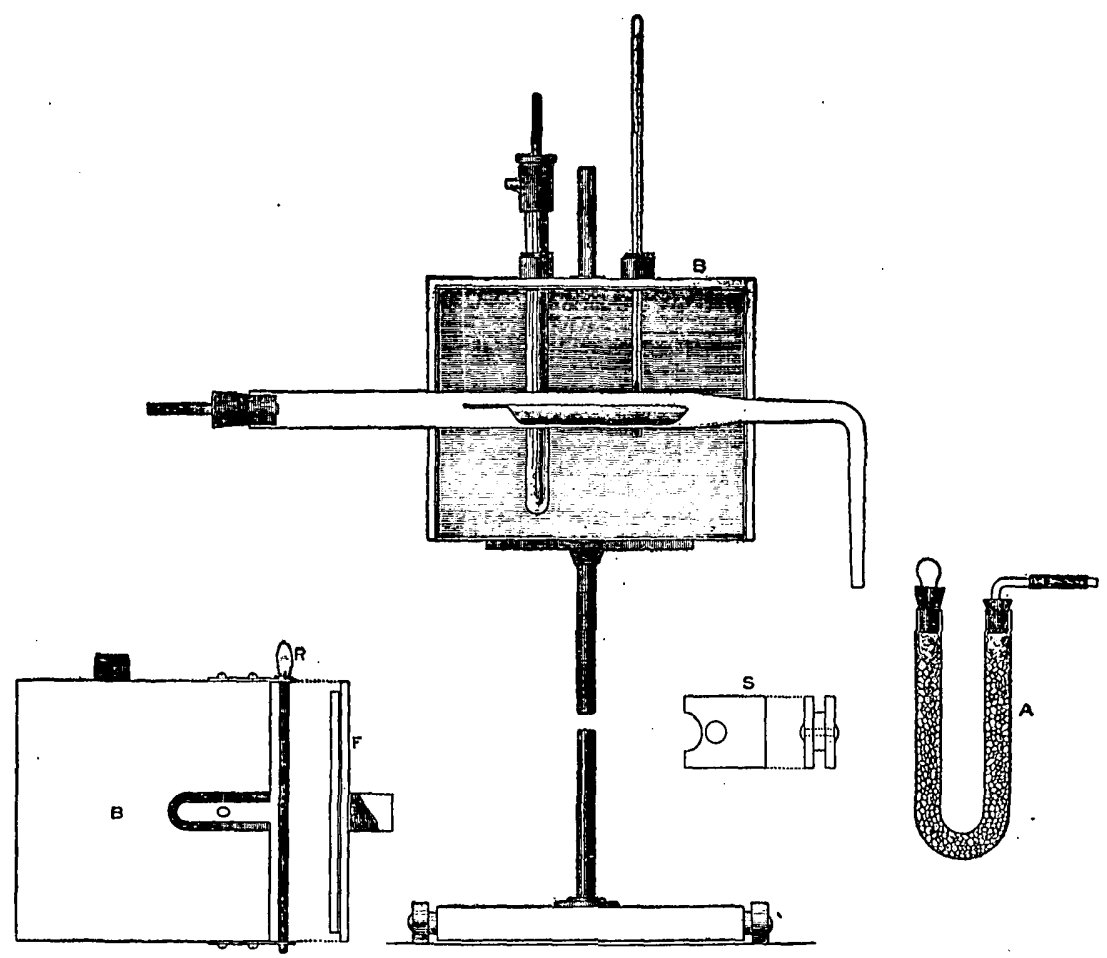

FIG. 3.-Chatard's form of drying oven for water determinations. B, copper box, $18 \mathrm{~cm}$. long, $10 \frac{1}{8} \mathrm{~cm}$. high, $9 \mathrm{~cm}$. wide, open in front, its sides and top covered with asbestos board; $\mathrm{s}$, two slides of different sizes to close the openings $O$, after the tube is in position; $F$, asbestos-board front stiffened by an interlaid sheet of copper; $R$, metal rod to hold front in place; $A$, calcium chloride absorption tube.

outer one, being slightly larger, by its projecting edges hinders the door from falling in and helps to prevent air currents. This door is held in place by the metal rod $\mathrm{R}$. The little slides $\mathrm{S}$ are made in a somewhat similar manner, and are intended to slip in from the front and close the two openings $\mathrm{O}$ after the tube is in place, but before closing the front.

For other forms of tubes adapted to similar determinations, see pages 40 and 46 . 


\section{WATER-TOTAL OR COMBINED.}

\section{ARGUMENTS AGAINST "LOSS ON IGNITION" METHOD.}

In a few cases the simple loss on ignition of a rock will give the total water with accuracy, but in the great majority there are so many possible sources of error that this old-time method can rarely be used with safety. Only when the rock is free from fluorine, chlorine, sulphur, carbon, carbon dioxide, and fixed oxidizable constituents can the loss be accepted as the true index of the amount of water present, and it is rarely that a rock is met with fulfilling these conditions, especially as to the absence of ferrous iron. Blast ignition in presence of carbon dioxide alone of the above list may give a correct result, after separate estimation of the carbon dioxide, provided this emanates from carbonates of the earths and not from those of iron or manganese. The long-maintained and still upheld idea that in presence of ferrous iron a sufficiently correct result is obtainable by adding to the observed loss an amount needed for oxidizing all ferrous iron is not justifiable. There can be no certainty that the oxidation has been complete, especially in the case of readily fusible rocks, and at the high temperature of the blast a partial reduction of higher oxides is not only possible but sometimes certain. The inability to insure complete oxidation by simple ignition is illustrated in the case of precipitated ferric hydroxide which has been ignited in contact with its filter paper. If the quantity was in any degree large it is sometimes decidedly magnetic, presumably from presence of magnetic oxide, which no amount of heating wholly oxidizes, especially in the larger grains. Neither is evaporation with nitric acid and reignition sufficient to destroy the magnetic property of the oxide, as has been claimed.

Direct weighing of the water evolved is then imperative in most cases, and of the numerous methods advocated, or in general use, several will now be considered.

DIRECT WEIGHING OF THE WATER WITHOUT THE USE OF ABSORPTION TUBES-PENFIELD'S METHODS.

For minerals easily deprived of their water.-If no other volatile constituents than water are present, the beautifully simple method first used by Prof. G. J. Brush and extended by Prof. S. L. Penfield ${ }^{1}$ leaves nothing to be desired for accuracy. It consists simply in heating the powder in a narrow tube of hard glass, enlarged at the closed end and provided with one or two further enlargements in the middle to hold the water and prevent its running back and cracking the hot glass. A capillary glass stopper fitted in with rubber tubing pre-

\footnotetext{
${ }^{1}$ Am. Jour. Sei., 3d series, Vol. XLVIII, p. 31, 1894; Zeitsch. für anorg. Chemie, Vol. VII, p. 22,
} 1894. 
vents loss of water by circulating air currents. The tube being held horizontally, the bulb is heated to any required degree by the Bunsen or blast flame. Moistened filter paper or cloth wound about the cooler parts of the tube insures condensation of all water. The heated end being finally pulled off, the tube is weighed after cooling and external cleansing, and again after the water has been removed by aspiration. For most rocks, as they contain little water, central enlargements of the tube are hardly needed.

Various forms of tubes used by Penfield are shown in fig. 4 .

Before using, even if apparently dry, "these tubes must be thoroughly dried inside, which is best accomplished by heating and aspirating a current of air through them by means of a glass tube reaching to the bottom."

How this simple tube is made to afford entirely satisfactory results

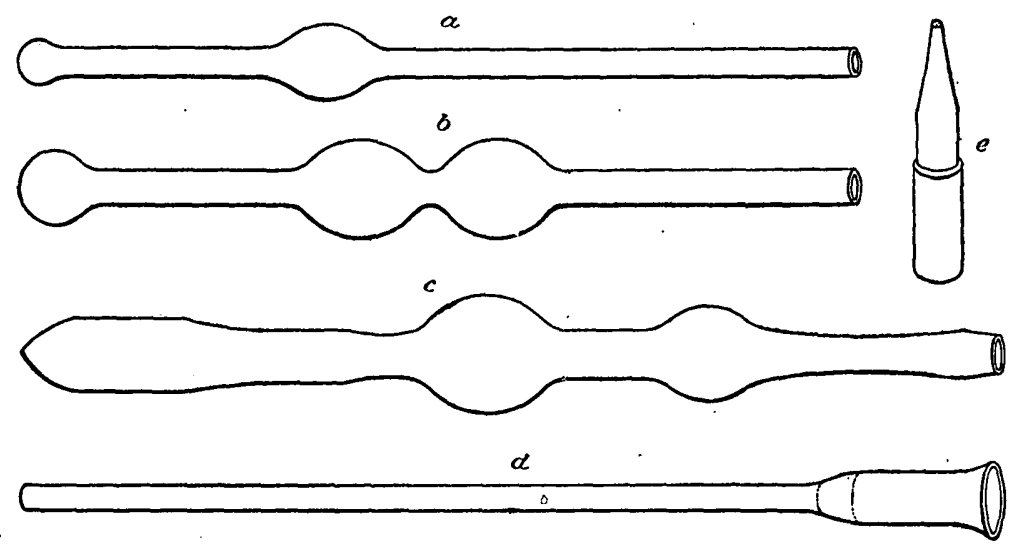

Fra. 4.-Penfield's tubes for water determination in minerals. $a, b, c$, different forms of tubes; $d$, thistle tube for introducing the powder; $e$, capillary-tipped stopper.

with minerals, even when carbonates are present, is fully set forth in the paper cited.

Few rocks, comparatively, are altogether free from other volatile constituents. Hence, for refined work the application of this apparatus in the simple manner above set forth is limited. It may, however, be used with the addition of a retainer for fluorine, sulphur, etc., in the shape of calcium, lead, or bismuth oxides.

For minerals not easily deprived of their water.-When minerals are present which do not give up their water wholly, even over the blast, as talc, topaz, chondrodite, staurolite, etc., Penfield's simple combination of fire-brick and charcoal oven, depicted in fig. 5, must be used, either with or without a retainer for fluorine, as circumstances demand. The part of the tube in the fire is to be protected by a cylinder of platinum foil tightly sprung about its end, and the part outside by asbestos board, as well as by wet cloth or paper. A piece of charcoal 
is likewise laid on the tube, as well as beneath and behind, and the blast flame is given a horizontal direction, so as to play upon the side of the apparatus. In this way a most iṇtense temperature can be reached.

In whichever way the apparatus may be used, the water found is

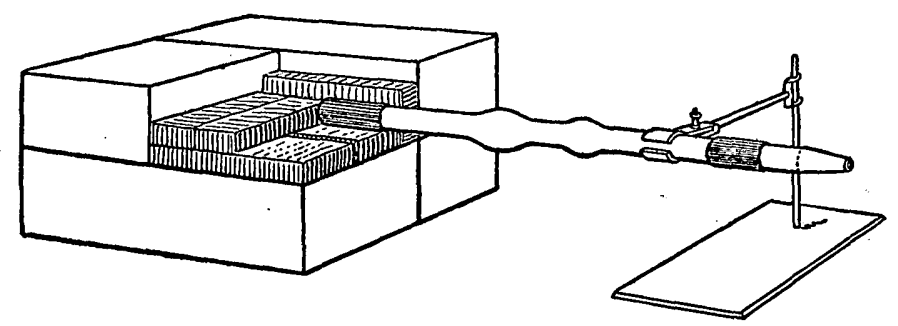

FIG. 5.-Penfleld's fire-brick and charcoal oven for use in determining water.

the total water, from which that found separately at $105^{\circ} \mathrm{C}$. may be deducted if desired.

\section{DIRECT WEIGHING OF THE WATER IN ABSORPTION TUBES.}

Penfield's procedure.-The simplest of these methods as to apparatus, and one permitting, by the use of auxiliary arrangements such $\approx \mathrm{s}$ are shown and described on page 37, the determination of the hygroscopic as well as any other fraction of the water, is the following glasstube arrangement (fig. 6) of Dr. Penfield's, ${ }^{1}$ whereby the brick and charcoal oven already referred to (fig. 5) comes again into play, but without the half brick shown in that figure.

The tube is of about $15 \mathrm{~mm}$. internal diameter, and is fitted with two platinum cylinders at $\mathrm{A}$, one inside, the other outside, where the heat exposure is to be most intense.

These are made from pieces of platinum foil, about $0.07 \mathrm{~mm}$. in thickness and 8 by $11 \mathrm{~cm}$. in diameter, which have been previously bent around glass tubes of such

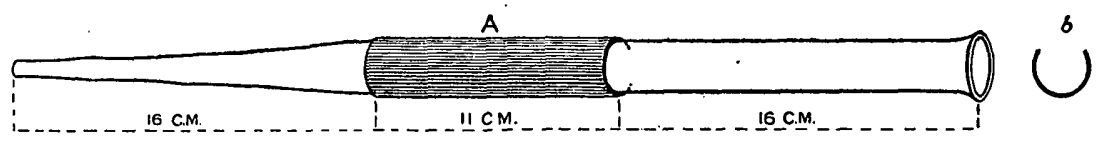

Fig. 6. -Tube for water determination according to Penfield. A, outer protecting covering of platinum foil. A second similar foil on the inside prevents the glass from collapsing when heated to softness. $b$, cross section of platinum boat.

a size that when applied to the combustion tubing the spring of the metal will hold them in place. A large platinum boat, 7 to $8 \mathrm{~cm}$. long and 11 to $12 \mathrm{~mm}$. in diameter, with a cross-section like $b$, should be used, since this will readily hold a gram of mineral mixed with 5 grams of sodium carbonate. *** The tube is placed in the angle formed by the charcoal lining, some pieces of charcoal are placed at the sides in front, leaving an opening through which the flame may be directed, and an additional piece is laid on top. The tube can readily be brought to a full white heat,

IAm.Jour. Sci., 3d series, Vol. XLVIII, p. 37, 1894; Zeitsch. für anorg. Chemie, Vol. VI, p. 22, 1894. 
and by forcing a slow current of dry air through the apparatus the carbon dioxide resulting from the decomposition can be removed and the water carried over into the weighed absorption tube. The glass fuses between the platinum casings, and in a number of experiments that have been tried there has not been a single instance where the glass tube has broken or shown any indication of breaking. After heating the tube will not crack if it is left to cool slowly on the charcoal, but it cannot be used a second time. *** At the high temperature to which the glass is subjected it of course becomes very soft and the ends must be properly supported; also the rubber connections and absorption apparatus must be carefully screened by asbestos board. By constructing a cover for the boat no material need be lost by spattering, and after making the water determination the contents may be used for the remainder of the analysis.

The inner cylinder of platinum serves to prevent the glass from collapsing as it softens, whereby distortion of the boat would result and its withdrawal for further examination of its contents would be impossible.

Gooch's apparatus.-Of more elaborate apparatus, designed to be used with fluxes, the tubulated platinum crucible invented by Dr. Gooch ${ }^{1}$ is capable of affording most excellent service, and it is the one by which far the larger number of water determinations in this laboratory have been made.

Fig. 7, which hardly needs detailed description, shows it in a modified form, which differs from the original forms of Gooch in that the tubes for connecting with both the drying and absorption vessels are constructed wholly of platinum instead of lead glass, the vertical one being bent horizontally at right angles for convenient attachment to the drying towers, and the side one also bent at right angles, but downward, and having its end slightly drawn in at $\mathrm{E}$ (fig. 7) so as to admit of easy insertion in the rubber stopper of a $U$-shaped calciumchloride tube as shown in fig. 9 (p. 44). With tubes of the lengths shown in the figure there is absolutely no danger of their ends becoming hot enough by conduction to scorch or soften the rubber stopper or other connection.

The extra first cost of the platinum extension to these tubes over the lead-glass ends of Gooch's original and modified forms need hardly enter as a factor into the question of employment of this apparatus. The glass ends often break, and only a rich lead glass, not easily obtainable, can be used, since it alone will not crack at the joint with the platinum after cooling. In its present form the whole apparatus weighs approximately 88 grams.

As an adjunct to its convenient use there is needed an ordinary upright iron ring-stand, with two small sliding rings, and a sliding ring-burner provided with entering ducts for gas and air blast. Across the uppermost ring there is an arrangement of stout platinum wire (S, fig. 8), forming at the center of the ring a secure seat for the 
upturned flange of the crucible proper. Both rings and burner can be clamped firmly at any height.

The rock powder, having been placed in the cylindrical crucible (C, fig. 7), is there mixed with not more than 3 or 4 grams of fully dehydrated sodium carbonate, ${ }^{1}$ or more of lead chromate if carbon is to be likewise determined. The crucible is sunk in its seat $\mathrm{S}$ (fig. 8) in the upper ring $R^{\prime}$ and the tubulated cap $T$ (fig. 7) is fitted on and attached to the calcium chloride drying towers-preceded by one containing potassium hydroxide if carbon dioxide is likewise to be estimated-on

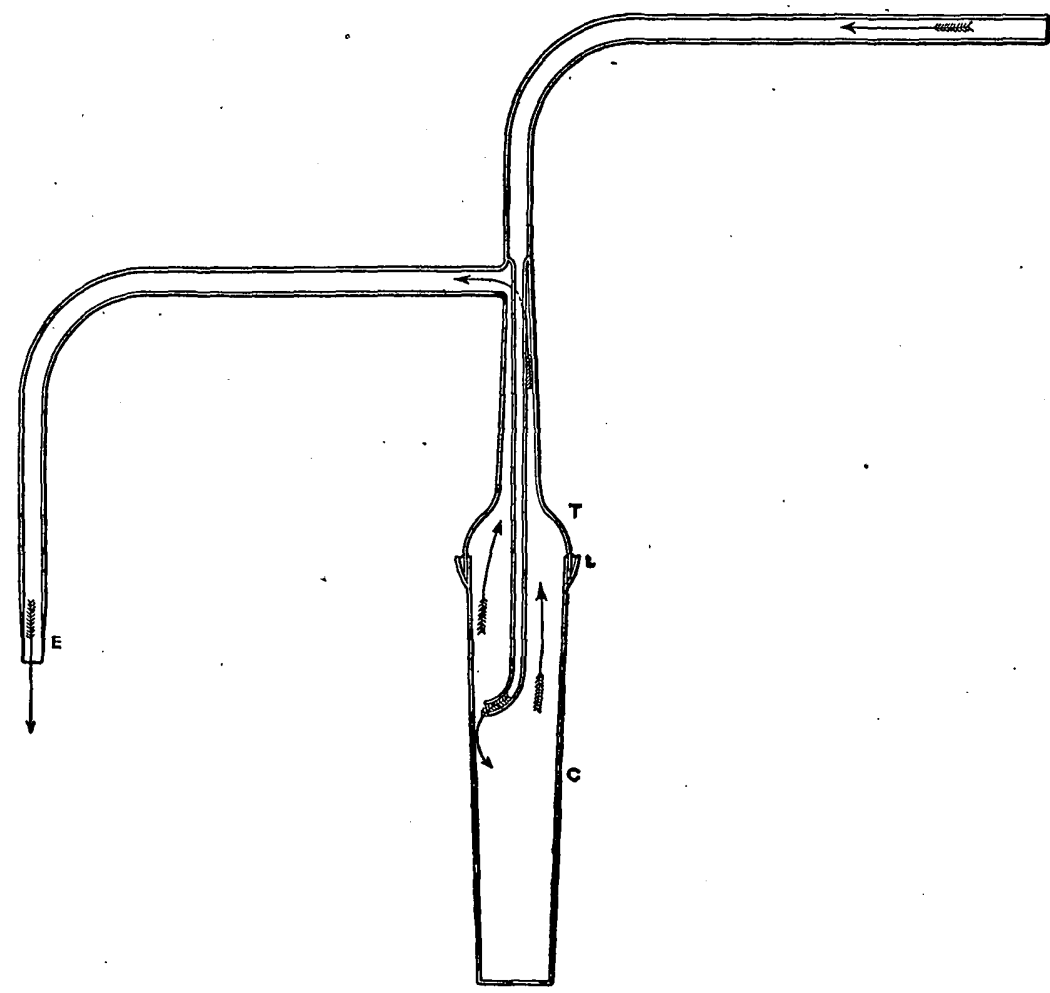

FIG. 7.-Modified form of the Gooch tubulated platinum crucible for the determination of water, one-half natural size. Weight about 88 grams.

the one side, and to a sulphuric-acid bulh tube B (fig. 9) on the other. Powdered sodium tungstate-free from arsenic, which would soon ruin the crucible lips - is now poured into the flanged lip L (fig. 7) in which the cap rests, and a metal vessel of cold water having been raised up by the lower ring $R^{\prime \prime}$ (figs. 8 and 9 ) until the platinum crucible is sufficiently immersed, the flame of an ordinary blast lamp is turned on to melt the tungstate. As soon as this is fused the flame is removed

1 This has been heated for a length of time to near its fusing point over a free flame or in an air bath, to decompose the bicarbonate it usually contains, and then placed in a desiccator. Thus heated it is not very hygroscopic. Penfield found that $2.5 \mathrm{grams}$ of it, spread out on a watch glass, gained only .0002 gram in 15 minntes. Potassium carbonate and potassium-sodium carbonate are too hygroscopie by far to be available. 
and the salt solidifies and makes an air-tight joint, the test of which is the permanence of the column of sulphuric acid in the bulb tubes caused by the contraction of the air in the platinum apparatus as it cools.

After drying by a current of air at $105^{\circ} \mathrm{C}$. for two hours, more or

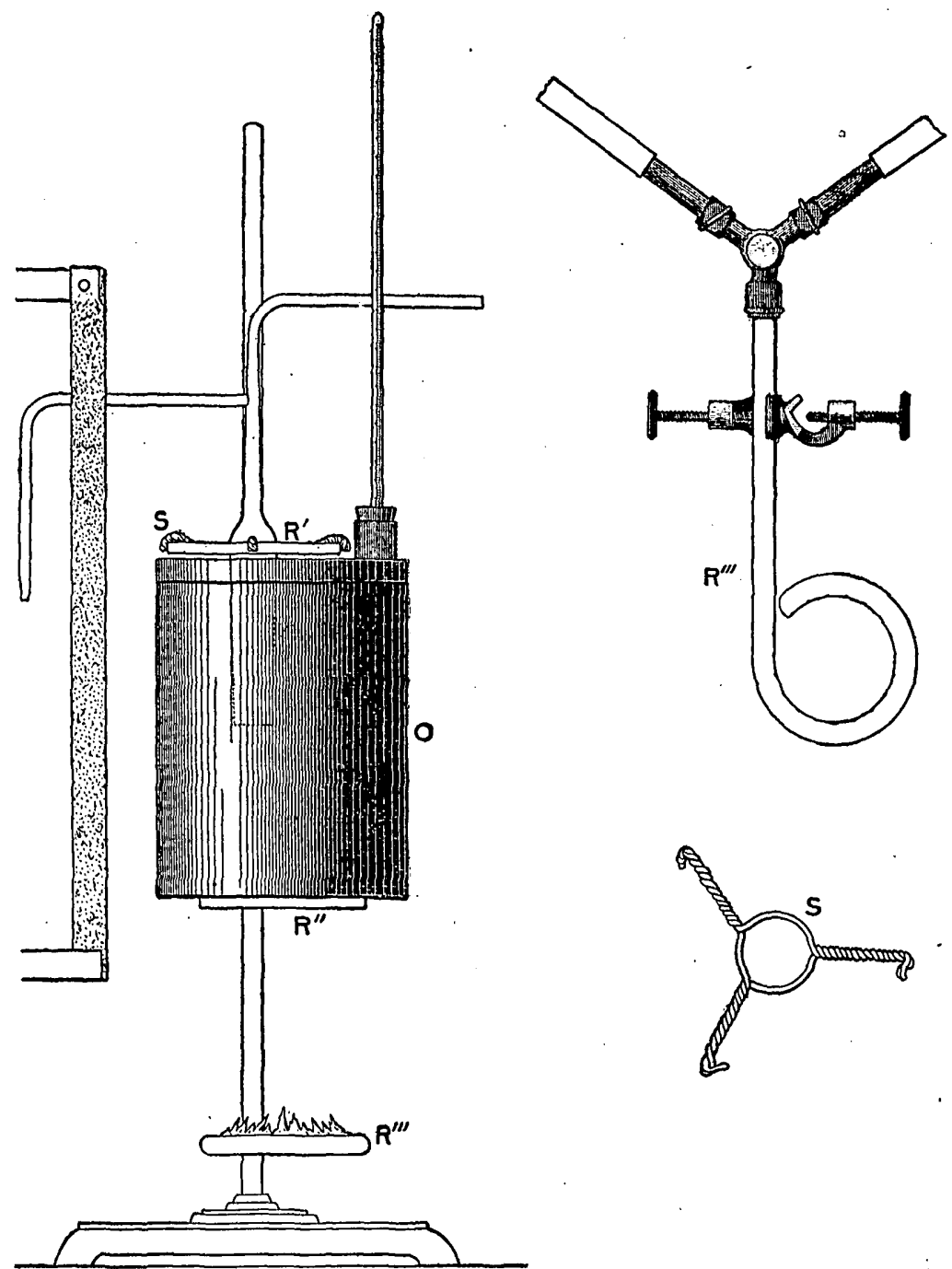

FIG. 8.-Details of the Gooch crucible for determining water. S, seat of stout platinum wire resting on ring $R^{\prime}$, and serving as a support for the crucible; $R^{\prime \prime \prime}$, blast-fed ring burner; $R^{\prime \prime}$, support for air or toluene bath 0 .

less (see below, p. 45), by means of an air or toluene bath as shown in figure 8, the absorption tube A (fig. 9) is interposed between the sulphuric-acid bulbs and the apparatus, being fitted to the latter by its stopper, which is at other times closed by a glass plug, and while a slow 
current of air continues to pass the gradual heating and subsequent fusion of the flux is brought about by the blast-fed sliding ring-burner $\mathrm{R}^{\prime \prime \prime}$ (figs. 8 and 9). The sodium-tungstate joint is shielded from the flame by small pieces of asbestos board $P$ (fig. 9), cut out so as to fit the crucible. When fusion is complete, as shown in the case of sodiumcarbonate flux by the decided slackening of the gas current through the safety bulbs attached to the drying tube, the flame is extinguished and a current of air is allowed to continue until the apparatus is cold.

This apparatus suffers from the drawback of being slightly permeable

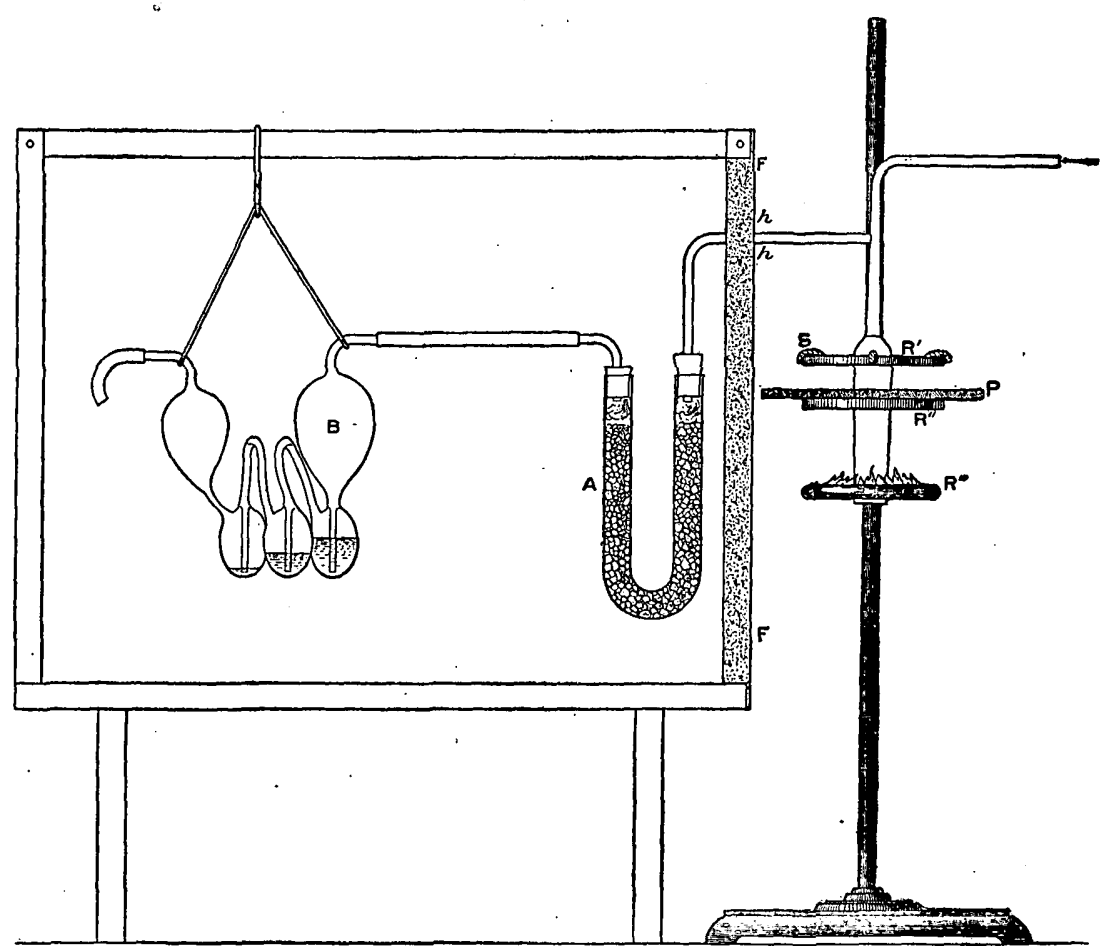

Fig. 9.-Arrangement during fusion of Gooch apparatus for determining water. $R^{\prime \prime \prime}$, blast-fed ring burner; $P$, protective asbestos-board shield resting on ring $R^{\prime \prime} ; F F$, board forming end of frame and covered with asbestos board to prevent being set on fire by the heat of the blast. This serves at the same time as an efficient shield for the absorption-tube $A$. In it there is bored a round hole at $h h$, through which passes the outlet tube from the crucible. B, sulphuric-acid bulbs serving to show the rate of gas current through the absorption-tube and at the same time to prevent back entry of moisture from the air into $\mathrm{A}$.

to combustion gases at high temperature. The defect can be overcome by causing the flame to play upon an outer ordinary platinum crucible, kept permanently filled with sodium-potassium carbonate. This protective crucible, however, is soon ruined for other purposes, being distorted by the alternate expansion and contraction of the carbonate.

It has been found that if the operation is carried out expeditiously and the final full heat applied for but a few minutes the error due to penetrating water gases is inappreciable. This hastening may be rendered safer by using rather finely powdered calcium chloride in the 
central section of the $U$-shaped absorption tube to avoid large anr channels. Through this, or any apparatus based on similar principles, the air current should always be forced, not drawn. A warm blast directed upon the exit tube near its entrance into the absorption tube greatly shoitens the time required and is to be recommended.

In this apparatus only the water expelled above $100^{\circ}$ to $110^{\circ}$ should as a rule be determined, and to effect drying of the mixed mineral powder and sodium carbonate, after luting the tubulated cap on the cylindrical crucible with sodium tungstate, the tube is sunk through a round hole in the cover into a small cylindrical air bath (fig. 8), which can be heated from beneath by the same ring burner which is subsequently to fuse the flux. A slow current of air is then forced through and the drying satisfactorily accomplished.

The reason why it is unsafe to attempt estimation of "hygroscopic" moisture in this apparatus is, that the luting of the two parts must be done by direct application of a flame to the tungstate, and considerable water vapor may enter the apparatus and be in part retained by the dried sodium carbonate.

Chatard's apparatus. - The platinum apparatus devised by Dr. Cha$\operatorname{tard}^{1}$ overcomes the permeability of the metal to gases and affords sharp results, moreover permitting of determining by direct absorption not only the hygroscopic water, but that which may be driven off at any desired temperature, either with or without fluxes. It is, however, perhaps even more costly than the Gooch apparatus, and the supposed nonliability to injury by warping, because of the protective layer of borax and asbestus, can hardly be considered as proved.

Merits of the above three forms of apparatus.-All of these apparatus, except the glass tube of the modified Brush method, permit of the estimation of other constituents besides water in the same portion if necessary, and by the use of lead chromate or potassium chromate, instead of sodium carbonate, graphite, or the carbon of organic matter, can be simultaneously determined with the water.

To one accustomed to its use, and with a drying and suspension attachment permanently set up, the Gooch apparatus, considering its limitations above set forth, offers perhaps the most handy and convenient means for the determination of water in rocks. Its high first cost, in comparison with the glass tube, is fully made up in time by its durability.

Jannasch's methods.-This zealous deviser of methods for mineral analysis has published in the Zeitschrift für anorganische Chemie and the Berichte der deutschen chemischen Gesellschaft several papers dealing with the problem of water determination in minerals, and in his text-book ${ }^{2}$ these are collected in more or less modified form.

${ }^{1}$ Am. Chem. Jour., Vol. XIII, p. 110, 1891; Bull. U. S. Geol. Survey No. 78, p. 84, 1891.

2 Praktischer Leitfaden der Gewichtsanalyse. Leipzig, von Veit \& Co., 1897. 
For the majority of silicates he finds dehydrated borax powder a most efficacious flux, usually at a very moderate temperature. The fusion is accomplished either in a platinum boat within a glass tube or in a tube of the form and dimensions shown in the accompanying fig. 10.

For rocks or minerals containing not much fluorine a retaining layer of granular lead chromate, or of previously fused and powdered lead oxide, is used as shown at $a$. Plugs of glass wool are used at $c, c$. Whether or not the boat is employed the borax is first introduced

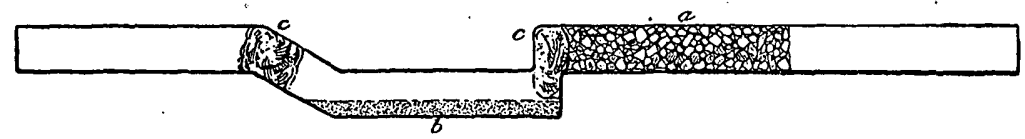

Fig. 10.-Glass tube for determination of water (Jannasch). $b$, mixture of mineral powder with borax; $c, c$, plugs of glass wool; $a$, layer of lead chromate or lead oxide. Total length of the tube, $33 \mathrm{~cm}$; inside diameter, $12-14 \mathrm{~mm}$.

and, together. with the retainer, is thoroughly dried out in an asbestos oven by a hot-air current. Then, after cooling, the mineral powder is added and thoroughly mixed with the borax. Heat is applied by a flat flame to the mixture, which soon melts and forms a clear fusion, when the action is complete. The blast may be used in extreme cases. The layer of retainer must be kept warm by an auxiliary·flame, and the absorption tube must be removed before the flame under the fused mass is extinguished, for the glass breaks as soon as this is done. Carbon dioxide can simultaneously be determined by attaching a soda-

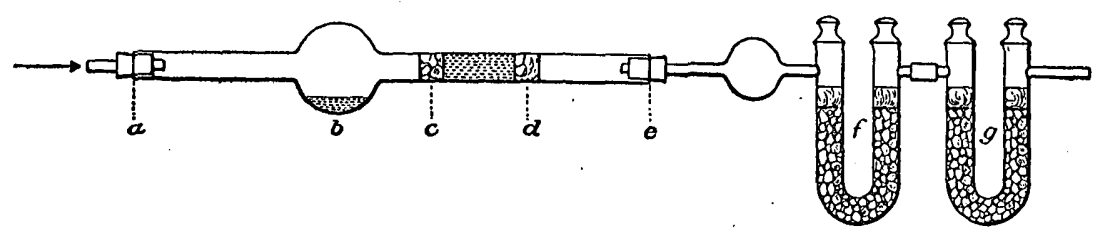

FIG. 11.-Glass tube fogr determination of water in special cases (Jannasch). Length from $a$ to $e$, $26 \mathrm{~cm}$; inside diameter somewhat over $1 \mathrm{~cm}$; volume of bulb $b, 25 \mathrm{~cm}^{3} . \quad c d$, retaining layer of lead oxide between plugs of glass wool; $f$, calcium chloride absorption tube; $g$, protective tube.

lime tube to the calcium-chloride tube. For one-half to 1 gram of silicate Jannasch uses $1 \frac{1}{2}$ to 2 grams of borax.

Regarding the borax method, its inventor insists upon the following points as essential to success, especially when the blast can not be applied: Most thorough mixing of flux and mineral powder and a most impalpable fineness of the latter.

The borax itself is prepared by heating pure crystallized borax in a platinum dish till a small portion has melted. That remaining unfused is powdered and again heated in the dish to dull redness for fifteen minutes, with constant stirring. The powder is placed in a tube with 
tightly fitting glass stopper and kept over sulphuric acid. It must not be kept long without reheating, because of being hygroscopic.

Another form of tube used by Jannasch for special purposes is shown in fig. 11. Minerals, such as topaz, which is not fully decomposed by the borax method and which contains a large amount of fluorine, are fused at $b$ with about six times their weight of lead oxide. A layer of lead oxide between $c d$ serves to retain any fluorine escaping from the fusion.

VI. SILICA, SEPARATION FROM ALUMINA, ETC.

\section{ALTERNATIVE METHODS OF DECOMPOSITION.}

PRELIMINARY REMARKS.

The practice of separating alumina, etc., by the usual methods, after first attacking the rock powder by hydrofluoric and sulphuric acids-silica being estimated in a separate portion-while attractive in principle, was abandoned by the writer after fair trial, owing to the disturbance sometimes occasioned by incomplete expulsion of fluorine and to a less degree by the presence of sulphates instead of chlorides. With exception of the comparatively few analyses made thus, the sodiumcarbonate method has always been employed. In the case of rocks rich in fluorine strict accuracy would require the separation of silica to be made as in the Berzelian method for fluorine estimation (see footnote, p. 50), but in practice it is not often necessary to resort to this tedious procedure, since the amount of fluorine is usually small and it can by no possibility cause a loss of much more than three-fourths its own weight of silica by volatilization as silicon fluoride when the sodium-carbonate fusion is evaporated directly with hydrochloric acid. Probably the loss is less, since some fluorine perhaps escapes as hydrofluoric acid. However this may be, the error is of comparatively slight importance, since it attaches to the constituent always present in greatest amount.

Various fluxes other than alkali carbonates have been recommended for breaking up silicates insoluble in ordinary acids, such as lead and bismuth oxides, lead carbonate, borax, and boric oxide. Professor Jannasch and his pupils have been especially active of recent years in this line of work, as evidenced by their numerous published papers. One of the advantages these fluxes possess over the alkali carbonates is their removability after serving their purpose, thus allowing the various separations to be made more perfectly and without the annoying interference of several grams of foreign fixed salts, which are most troublesome in that part of the analysis devoted to the separation of silica, alumina, iron, lime, and magnesia. 
Another of their advantages is that with some of them it is possible to estimate in one portion the alkalies, in addition to those constituents usually determined in the silica portion. Where the material is limited, as it so often is in mineral analysis, this is a most important advantage, sufficient to outweigh all possible objections; but in rock analysis, where the supply of material is usually ample, it is rarely worth considering. A still further point in their favor is that it is probably more easy to obtain them entirely free from fixed impurities than an alkali carbonate.

There are, however, objections to their use. With some of them an extraordinary amount of time must be devoted to grinding the mineral to an impalpable powder, and the flux itself may need considerable hand pulverization. Once introduced, they must be removed before the analysis can be proceeded with, and this removal takes much time and is always a possible source of error.

In mineral analysis these objections are entitled to far less weight than in rock analysis, since the object sought-usually the deduction of a formula-warrants the expenditure of much time and painstaking care. Finally, it has been found that one or more of these fluxes are not available for altogether general use, since certain minerals do not fully succumb to their attack under simple conditions, as andalusite with boric oxide and others with lead oxide (Jannasch). Therefore, however well adapted one or the other of these methods may be for the analysis of homogeneous minerals, it is very improbable that the vivid anticipations of Professor Jannasch, to the effect that the boric-oxide method will soon supersede the alkali-carbonate-fusion method in rock as well as in mineral analysis, will be speedily realized. Nevertheless, the boric-oxide-fusion method, owing to its evident merit, will be described in detail after brief reference to a means of bringing refractory silicates into solution without employing any solid reagent.

DECOMPOSITION OF REFRACTORY SILICATES BY FYDROCFLORIC ACID UNDER PRESSURE.

Jannasch ${ }^{1}$ pours upon the finely ground rock powder contained in a platinum tube of about $26 \mathrm{~cm} .^{3}$ capacity a somewhat diluted hydrochloric acid (4 acid to 1 water), places over the open end a cap which does not hermetically close the tube, inserts the latter in a larger one of potash glass likewise partially filled with the diluted acid, seals the glass tube, and places it in turn in an inclined position in a steel Mannesmann tube containing ether or benzine to equalize the pressure, and heats to any desired temperature up to $400^{\circ} \mathrm{C}$.

The chief drawback seems to be a somewhat incomplete decomposition, doubtless due to the necessarily inclined position of the tube, which causes the powder to collect at the lower end, and thus renders

1 Ber. deutsch. chem. Gesell., Vol. XXIV, p. 273, 1891, and Zeitschr. für anorg. Chem., Vol. VI, p. 72, 1894. 
decomposition less complete than if the material were spread evenly throughout the length of the tube. Further, the acid strongly attacks the platinum unless the air in both the platinum and the glass tubes is replaced by carbon dioxide. Even when this is done, several milligrams of platinum are found in the silicate solution.

Nevertheless, to those possessing the necessary platinum and steel tubes the method can render efficient service in special cases when economy of material is imperative.

THE BORIC OXIDE METHOD OF JANNASCH AND HEIDENREICH. ${ }^{1}$

Preparation of the boric oxide.-This demands, if the alkalies are to be estimated in the same portion as silica, etc., an absolutely alkalifree boric acid, which can be prepared by two or three recrystallizations of a good commercial article. The purified crystals are dehydrated and fused in a large platinum crucible. This is then suddenly cooled to cause the anhydride to crack into pieces of a size convenient for powdering, which are to be kept in a tight glass and powdered as needed, since the anhydrous oxide is hygroscopic.

Treatment of easily decomposable silicates.-To this flux Jannasch and Heidenreich find that nearly all silicates readily succumb over the ordinary blast lamp. The fusion is made in a large crucible holding $40-65 \mathrm{~cm} .{ }^{3}$, and the proportion of flux to be used is gauged according to the nature of the silicate, ranging from 3 to 8 and more parts to 1 of mineral. This last must be finely powdered, especially the most resistant, the authors recommending the expenditure of one-half to one hour's time for the grinding of one-half to 1 gram of powder. A low burner heat is applied for five to ten minutes till water is expelled, which is then gradually increased till the gas is fully turned on. Bubbling and rising in the crucible is prevented as far as possible by using a short platinum rod which does not reach above the edge of the crucible. When the mass has been in quiet fusion for a time in the covered crucible the blast flame is applied. The average duration of the entire operation is twenty to thirty minutes, but depends much on the character of the mineral.

Treatment of refractory silicates.-For those minerals which, like andalusite, cyanite, and topaz, are not fully decomposable by the heat of the ordinary blast flame, Jannasch and Weber ${ }^{2}$ use a flame fed by oxygen instead of air. The blast lamp, of $2 \frac{1}{2} \mathrm{~mm}$. opening, is supplied with gas from at least five or six ordinary gas cocks, and the flame is made broad and free from luminosity. The mineral having been first heated as above described, but with a much larger "proportion of flux-as high as 30 to $1-$ a few grams additional of boric oxide 
are added and the oxygen blast is applied till, in ten or fifteen minutes, the fusion is as transparant as glass. ${ }^{1}$

Further treatment after fusion. - From this point the further treatment is the same in both cases, and as modified by Jannasch and Weber (loc. cit.) is as follows:

The hot crucible is cooled in cold water and the contents are turned into a very large porcelain or platinum dish, to which, after covering with a glass, a saturated solution of hydrochloric acid gas in methyl alcohol is added. ${ }^{2}$ The cover being then removed, the liquid is heated to boiling over asbestos board by an inch-high flame, stirring constantly, or it is left without attention over a lower flame or on a water bath heated short of boiling. The crucible is cleansed in a similar manner, and its contents are added to the dish. In ten to fifteen minutes, with occasional addition of the methyl chloride, solution is complete and the liquid is then boiled down to a small volume and evaporated to dryness on the bath. The residue is then digested on a bath at $80^{\circ}$ to $85^{\circ} \mathrm{C}$. three or four times in succession, with the ether solution, in order to remove the last traces of boron as boric ether. Care should be taken to wash down from the sides of the dish, with methyl-chloride solution, the boric acid formed and deposited thereon during the evaporation.

Possible objections to the boric-oxide method.-Very much is claimed by Jannasch for this method, but with all its undoubted merit there are two points which may militate against it in time. The boric ether, driven off in such enormous quantities, at once decomposes in contact with moisture, and boric acid settles over all objects with which it comes in contact. The hood must become thickly. coated. Hence a special hood for these evaporations alone seems to be called for, otherwise boric acid may at any time fall into other dishes and cause untold trouble. The second objection attaches to the use of the oxygen flame when alkalies are to be estimated in the fusion, and the ability to so determine them is one of Jannasch's chief claims in favor of the method, for it can not be doubted that at the high temperature of this flame alkalies are volatilized in part. Borax can be slowly but wholly volatilized over the ordinary blast, hence there is great reason to fear sufficient loss at this_much higher temperature to give rise to serious error at times.

THE SODIUM-CARBONATE METHOD.

Purity of the sodium carbonate used as a flux.-Notwithstanding the most earnest efforts for years, it has been impossible to procure, either

\footnotetext{
${ }^{1}$ An interesting and important observation reported by Jannasch and Weber is that when the oxygen blast has been used for silicates carrying fluorine or mixed with fluorides, the fluorine seems to be wholly expelled as boric fluoride, without loss of silica. If this should prove to be generally true, an easy way is at last afforded for estimating silica in such cases, where even its detection, when present in small amount, has heretofore been difficult.

2 Made by passing dry $\mathrm{HCl}$ into cooled $\mathrm{CH}_{4} \mathrm{O}$ for from one to two hours.
} 
in the open market or by special arrangement with manufacturers, an article of sodium carbonate which can be called chemically pure. With special precautions small lots can be prepared in the laboratory that will contain less than 1 milligram total impurity in 10 grams; but such an article can not be purchased in the market, and rarely will the so-called chemically pure dry sodium carbonate contain as little as 1 milligram in 10 grams. The invariable contaminating substances, aside from sand and straw, which bave sometimes been found in large amount, are silica, alumina, iron, lime, and magnesia, all of these going into aqueous solution with the carbonate. The chief of these impurities are usually silica, alumina, and lime. An article of the above degree of purity is satisfactory in almost all imaginable cases, since the use of the usually extravagant amount of 10 grams for a fusion would introduce an error of but 0.1 per cent in the analysis, supposing 1 gram of mineral to be operated on, and it would, moreover, be distributed over several constituents. This error is undoubtedly fully equaled by the introduction of dust from the air in the various long evaporations.

Precautions in fusing.-Special directions with regard to the fusion and its first treatment are unnecessary, except to say that ordinarily from 4 to 6 parts of flux should be used to 1 of rock and that the flame should not be directed vertically against the bottom of the crucible, but at an angle against the side and bottom, nor should the flame be allowed to envelop the whole crucible. These precautions apply in all ignitions of reducible substances, and yet they are rarely observed. In neither case, if neglected, will there be the necessary oxidizing atmosphere within the crucible; on the contrary, reduction may occur fraught with serious consequences. This is especially true if the rock contains more than traces of pyrite or other sulphide, when, after cleansing and igniting the crucible, there may appear on its interior a darkening due to oxidation of reduced iron which had alloyed with the platinum. This may in exceptional cases amount to several milligrams in weight, and can be removed only by repeated ignitions, followed each time by scouring or treatment with hydrochloric acid or acid potassium sulphate. In order to avoid the use of niter in case of pyritiferous rocks, it is well to first roast the weighed powder in the crucible in which the fusion is to be made.

Treatment after fusion.-When fusion is complete, the crucible is seized with the tongs (fig. 1, p. 23) and the contents are caused to solidify in a thin sheet over the sides and bottom by imparting an appropriate rotating motion with the arm during the cooling process. This is far preferable to allowing the melt to form a thick cake at the bottom, since. much less time is required for disintegration, and separation from the crucible is usually much easier.

It sometimes happens that the cooled flux, and even its solution, 
will indicate absence of manganese when it is really present in quantity to give normally a strong coloration. Two fusions made side by side or successively, under apparently similar conditions, may in one case show little or no manganese, in the other considerable. This observation has been frequently made, and therefore the absence of a bluish-green color in the fusion is not to be taken as proof of the absence of manganese. This difference of behavior I can ascribe to no other cause than that of a reducing atmosphere in one of the crucibles and an oxidizing one in the other, even though the conditions were apparently alike.

The contents of the crucible are placed in a rather tall covered beaker with some water, and hydrochloric acid of 1.1 specific gravity is added in excess. The depth of the pink color usually produced on addition of the acid allows of judging approximately as to the amount of manganese present. The beaker is placed on the water bath, and when disintegration is complete, having been assisted by gentle pressure with a blunt glass rod, the contents are transferred to a large platinum dish and evaporated on the bath.

\section{SUBSEQUENT TREATMENT.}

From this point the treatment will ordinarily be the same whether the boric-oxide or the sodium-carbonate method of decomposition has been employed.

Drying and testing of sitica.-As to the best way of rendering silica insoluble by evaporation, the writer's predilection is for a double evaporation instead of a single one on the water bath. By fusing with sodium carbonate in the forenoon, the silica is ready for the first filtration in the afternoon. It is quite unnecessary to carry the evaporation beyond approximate dryness. The filtrate is again evaporated, always in platinum, and is ready for final filtration the following morning, when approximately 1 per cent of silica is recovered and added to the main portion. The writer's experience is that a better separation of silica is effected hereby, and in no more time than by a single long evaporation. That which is subsequently recovered from the precipitate of alumina, etc. (p. 56), rarely exceeds a half or, at the most, 1 milligram.

Drying in an air bath at $110^{\circ} \mathrm{C}$. or higher, or on a hot plate or sand bath, or over a free flame, in order to render silica insoluble, offer's no advantage unless much magnesium is present, and then the most favorable temperature, according to Gilbert, ${ }^{1}$ is $120^{\circ} \mathrm{C}$. The presence of much calcium chloride seems to facilitate dehydration of the silica, while magnesium chloride above $120^{\circ} \mathrm{C}$., on the other hand, by decomposing, forms a silicate which dissolves in hydrochloric acid and

${ }^{1}$ Technology Quarterly, Vol. III, p. 61,1890. Abstract in Fresenius's Zeitschr. für anal. Chemie, Vol. XXIX, p. 688, 1890 . 
increases the amount of silica carried into the filtrate. It does not appear from Gilbert's paper that the blast-furnace slags, on which he experimented, contained titanium, phosphorus, or iron in appreciable amounts. Basic magnesian rocks usually do, and in such cases it is probable that the employment of a drying temperature of $1.20^{\circ}$ would materially add to the large impurity always to be expected with the silica. In other cases he confirms the earlier belief that drying temperatures higher than that of the water bath increase the amount of insoluble impurity, chiefly alumina, in the silica, and that this amount can not be reduced by long digestion with hydrochloric acid. Further, he confirms Lindo's statement that evaporation with sulphuric acid till the appearance of white fumes gives a higher result in silica than with hydrochloric acid. But for general rock analysis the use of sulphuric acid at this stage must be rejected utterly.

Blasting for twenty to thirty minutes ${ }^{1}$ is necessary to expel all moisture from the silica, and it is then not hygroscopic. Its weight should always be corrected for impurities, which are never absent, by evaporating with hydrofluoric and sulphuric acids and again blasting. If toward the end of evaporation with these acids, when the hydrofluoric acid has been driven off and the sulphates begin to appear in solid form, the residue has a peculiar milky or enamel-like appearance, it may be taken as evidence of much phosphorus and titanium. This appearance is possibly due to zirconium with the phosphorus and titanium, ${ }^{2}$ and is so unusual and striking that it is worth while calling attention to it. With basic rocks very rich in titanium and phosphorus the residue may amount to 2 or even 3 per cent of the rock.

The subsequent precipitate of alumina, etc., is usually ignited in the crucible containing the residue from the silica.

It might be supposed that this residue would contain most of the barium of those rocks carrying that element, together with sulphur or sulphates, but the reverse is true as a rule. Only when there is a considerable excess of $\mathrm{SO}_{3}$ over the $\mathrm{BaO}$ will much of the latter be found there, and in the vast majority of cases there is none at all. Should some be present, its removal and estimation at this stage is not necessary, as it can be more conveniently recovered later, together with the silica accompanying the alumina, etc., precipitate (p. 56).

The separation of silica in rocks containing fluorine has been touched upon in commenting on the boric-oxide and sodium-carbonate methods of fusion, ${ }^{3}$ and will be considered further under the head of Fluorine (p. 103).

I It must be borne well in mind that some platinum crucibles lose weight steadily and very appreciably on long blasting, not only when new but even after long use. When a crucible suffers from this defect the rate of loss should be ascertained from time to time and allowance made accordingly, or else the weight of the crucible should be taken after and not before ignition of the precipitate. (Sec on this sulject Hall, Jour. Am. Chem. Soc., Vol. XXII, p. 494, 1900.)

3 See first footnote, p. 57.

${ }^{3}$ See p. 47 and footnote, p 50. 
Platinum in filtrates. - The filtrates from the silica always contain notable amounts of platinum. This arises in very small degree from the crucible fusion, in a larger one indirectly from the action of hydrochloric acid on manganate, vanadate, and sometimes chromate. of sodium, and, if much iron is present, in no small degree from the reduction of ferric chloride to ferrous by the platinum of the dish. This last reaction is little known, apparently, but is mentioned in Gmelin-Kraut, ${ }^{1}$ and can be readily demonstrated by evaporation of ferric chloride in platinum.

The removal of this platinum before precipitating alumina and iron is not necessary (but see second footnote, p. 57), and to do so involves the reoxidation of all iron and subsequent boiling to remove or destroy the excess of oxidizing agent, together with the expenditure of much valuable time. The iron is already oxidized by the fusion, and needs no further help in that direction. Nevertheless, if time is not a prime object, its removal by hydrogen sulphide is to be recommended. In the following descriptions, however, it is assumed that the platinum has not been gotten rid of at this stage.

\section{METALS PRECIPITAPABE BY HYDROGEN SULPHIDE.}

The presence in appreciable amounts of metals precipitable by hydrogen sulphide, except perhaps copper, is of such infrequent occurrence in most rocks that discussion is unnecessary in their connection. In case it is necessary to precipitate them at this stage, however, it is always well to bear in mind that a little titanium may be thrown down along with them. Separations of the silica should be made in porcelain, to eliminate platinum, or, better still, the quantitative estimation of these metals should be made in a separate portion of the rock broken up by the action of hydrofluoric and sulphuric acids.

\section{ALUMINUM. TOTAL IRON.}

\section{INDIRECT METHOD FOR ALUMINUM.}

The common practice in this laboratory is to find alumina by difference, after deducting from the precipitate produced by ammonia or sodium acetate the sum of all other oxides this precipitate may contain. Of these, only ferric oxide, titanic oxide, and the trace of silica are determined in this portion (see also second footnote, p. 57), those of phosphorus, vanadium, chromium, and zirconium being looked for in other portions of the rock powder. This throws upon the alumina all errors involved in their separate determinations; but these may balance, and

${ }^{1}$ Anorg. Chem., Vol. III, p. 359. Sixth revised edition. 
in any case the probable error can hardly be as high as that involved in the direct weighing of the alumina itself, considering the difficulty of effecting a satisfactory separation of it from all the other admixtures, an operation which would, moreover, immoderately extend the time required for each analysis.

\section{PRECIPITATION OF ALUMINUM, IRON, ETC.}

Precipitation by ammonia.-Two precipitations by ammonia at boiling heat are usually quite sufficient to separate iron, aluminum, phosphorus, vanadium, chromium, titanium, and zirconium, if all these are present, from nickel, manganese, the alkaline-earth metals, and magnesium, provided ammoniacal salts are present in sufficient quantity. This last point is of special importance as regards magnesium, and failure to observe it is doubtless the reason why many old analyses, and sometimes modern ones, show utterly improbable percentages of alumina, especially as chemists were formerly often satisfied with a single precipitation. The necessary ammonium chloride is better obtained by the use of purified ammonia water and hydrochloric acid than by the addition of the solid salt, which is seldom pure.

Precipitation by the basic acetate method.-But it will occasionally happen that the separation from even very small amounts of manganese is altogether incomplete, and the uncertainty of insuring this separation has led the writer of late to employ the basic acetate method for the first precipitation in all cases where manganese is present-and the exceptions are few-even though the precipitation of alumina is sometimes less complete than by ammonia, and in spite of other admitted defects, as, for instance, a tendency of the precipitate to run through the filter on washing. ${ }^{1}$ Not more than 2 , or at most 3 , grams of sodium acetate need be used. After slight washing and sucking dry at the pump, the precipitate is redissolved in a large excess of hydrochloric acid and reprecipitated by ammonia in slight excess. The complete boiling off of this excess is unnecessary, as pointed out by Genth and Penfield, since it is apparently the washing with pure water and not the free ammonia which carries small amounts of alumina into the filtrate. Penfield and Harper ${ }^{2}$ recommend washing with a dilute solution of ammonium nitrate $\left(20 \mathrm{~cm} \cdot{ }^{3}\right.$ nitric acid, neutralized by ammonia, to the liter), and also the solution of the first precipitate in nitric instead of hydrochloric acid, in order to shorten the washing, there being no chloride to remove.

\footnotetext{
1The fact must not be overlooked that certain of the rare earths may pass completely into the filtrate if the basic acetate method is followed. If then, later, on rendering the combined filtrates ammoniacal, an unexpectedly large precipitate appears, this should be carefully examined as to its nature. In an analysis of piedmontite from Maryland over 2 per cent of rare earths, including cerium and others not identified, were quantitatively separated in this way from iron, alumina, etc.

${ }^{2}$ Am. Jour. Sci., 3d series, Vol. XXXII, p.112, 1886.
} 
The filtrates are strongly concentrated separately ${ }^{1}$ in platinum, a drop or two of ammonia being added toward the end to the second one, and filtered successively through the same small filter into a flask of 150 to $200 \mathrm{~cm}^{3}$ capacity, the ammoniacal filtrate serving as wash water for the first dish and containing enough ammoniacal salt to prevent precipitation of magnesium in the first filtrate when mixed with it. If manganese has been deposited upon the surface of the dish it is removed by hydrochloric and a drop or two of sulphurous acids, which mixture is then passed hot through the filter. A reprecipitation by ammonia is then made, and the precipitate collected again on the filter and added to the main one, the filtrate passing into the flask containing the previous one. If much manganese is present, of course a second precipitation by ammonia of the small precipitate may be required. In these cases there is no difficulty in getting all the manganese into the filtrate.

IGNITION OF THE PRECIPITATE.

The combined precipitates of alumina, etc., are ignited moist, in the paper, unless considerable iron is present, when the main one is dried, removed as far as possible from the paper, and the latter ignited separately to prevent partial reduction of a portion of the iron, which can not then be wholly reoxidized by heating or by treatment with nitric acid (see p. 38).

Alumina in the quantities ordinarily found can not be fully dehydrated by the full heat of the Bunsen burner. It must be blasted for five or ten minutes. If iron is present in large amount this last operation must be conducted so as to insure free access of air to the crucible (p. 51).

RECOVERY OF SILICA AND POSSIBLE BARIUM IN THE ALUMINA PRECIPITATE.

The precipitate is dissolved by fusion with acid potassium sulphate, an operation which is accomplished without trouble in from two to four hours if the temperature is kept low and the acid salt has been properly made free from water and excess of acid. The melt is taken up with hot water and considerable dilute sulphuric àcid, the residue collected, weighed, and corrected by hydrofluoric and sulphuric acids for silica, which, as said before, rarely amounts to 1 milligram in weight, and further examined for barium (see p. 53) by dissolving any

\footnotetext{
IIf, instead of sodium acetate, ammonia alone has been used to precipitate alumina, etc., it has sometimes happened in the experience of others than the writer that on concentration of the first filtrate a pale straw-colored precipitate appeared, which remained on the filter with the traces of alumina thatimay also separate, although it is slowly soluble in hot water. This is said to be some compound of platinum, and attention is called to $\mathrm{dt}$ here for the guidance of others who may notice it and be unaware of its character.
} 
remaining residue in hot, strong sulphuric acid and diluting with cold water. ${ }^{1}$

ESTIMATION OF IRON IN THE PRECIPITATT OF ALUMINA, E.'C.

Without regard to the presence of vanadium.-The filtrate obtained in the preceding paragraph is reduced, hot, by hydrogen sulphide, boiled to collect sulphur and the platinum sulphide ${ }^{2}$ resulting from the bisulphate fusion, the hydrogen sulphide being allowed to pass for a short time after boiling. It is then filtered ${ }^{3}$ hot into a flask attached to a carbonic-acid apparatus and brought to boiling to expel hydrogen sulphide. When this is fully effected the flask is cooled in water while the carbon dioxide still passes, and the solution is then titrated by potassium permanganate. The results are strictly accurate, with the limitations set forth in the paragraph below, when care is taken with the reduction by hydrogen sulphide. The method is altogether superior to that involving the use of zinc, since no foreign impurity affecting the result is introduced and the ever-present titanium is not affected, nor is vanadium reduced below the condition of $\mathrm{V}_{2} \mathrm{O}_{4}$, whereas nascent hydrogen converts it, in part at least, to $\mathrm{V}_{2} \mathrm{O}_{3}$. Titanium can be conveniently estimated by adding hydrogen peroxide to the titrated iron solution (see p. 68).

:Some years ago, in a series of analyses of rocks from the Leucite Hills, in Wyoming, there was obtained at this stage, when it was customary to dissolve the melt in cold water preliminary to precipitation of titanium by boiling the neutralized sulphuric solution in presence of sulphur dioxide, a white, more or less flocculent residue amounting to 1 to 3 per cent of the rock, which was at first taken to be a mixture of tantalic and columbic acids. Eventually it was found to consist apparently of nothing but $\mathrm{TiO}_{2}$ and $\mathrm{P}_{2} \mathrm{O}_{6}$, with perhaps a little $\mathrm{ZrO}_{2}$. By repeated fusion with acid potassium sulphate and leaching with cold water it could be gradually brought into solution. It was these rocks which furnished the most striking instance of the peculiar, milky, sulphate residues mentioned on p. 53, as derived from the ignited silica.

Knop (Zeitschr. für Kryst., Vol. X, p. 73, 1885) seems to have obtained a similar mixture in analyzing minerals from the Kaiserstuhl in Baden, but its nature was not ascertained, though suspected to be, if not silica, columbiferous titanic acid.

2It may be mentioned that the precipitation of platinum from a hot sulphate solution is far quicker and cleaner than from hydrochloric acid. Further, this platinum sulphide, when ignited in the crucible in which the bisulphate fusion was made, should weigh together with the crucible itself what the latter weighed before the main silica precipitate was ignited in it; in other words, the weight of the platinum recovered by hydrogen sulphide should equal the loss in weight of the crucible due to attack by the bisulphate. In somewhat rare instances this will not be so, but the weight will be greater, showing a gain in platinum which may amount to a milligram. Tests have shown that this is not due to retention of platinum by the main $\mathrm{Al}_{2} \mathrm{O}_{3}$, etc., precipitate; hence, it must come from platinum mechanically loosened from the dish during the drying and powdering of the silica preparatory to its collection on the filter, or to some insoluble compound of platinum formed during evaporation and drying of the silica. It may also be in part or wholly due to contaminution from reduction of platinum during evaporation of the filtrate from the basic acetate separation. It will be remembered that from this filtrate a small amount of iron and alumina is recovered and added to the main precipitate. Hence it is always well in fine work to collect the sulphide and weigh the platinum in the original crucible, deducting any excess from the alumina, or else to get rid of the platinum by hydrogen sulphide before proceeding to the precipitation of alumina, etc. (see p. 54).

${ }^{3}$ Filtration is not necessary if only precipitated sulphur and no sulphides are in suspension, since this is without reducing action on cold permanganate solution, as Wells and Mitchell, and others before them, have pointed out. The above authors used this method of reducing ferric ironin titanic iron ores. (Jour. Am. Chem. Soc., Vol. XVII, p. 78, 1895; also Chemical News, Vol. LXXIII, p. 123, 1896.) 
Having regard to the presence of vanadium. - If vanadium is present the value found for iron will be in error by the amount of permanganate required to oxidize $\mathrm{V}_{2} \mathrm{O}_{4}$ to $\mathrm{V}_{2} \mathrm{O}_{5}$. The amount of the correction will differ according as titration of the iron is made after reduction by hydrogen sulphide or by nascent hydrogen. If the former is used, as should always be the case, because of the ever present titanium; the vanadium is reduced by it to $\mathrm{V}_{2} \mathrm{O}_{4}$, which in its action on permanganate is equivalent to two molecules of $\mathrm{FeO}$, while the reduction goes further with hydrogen. After the first transitory pink blush throughout the liquid, the slower-acting vanadium may require the addition of a drop or two more of permanganate before a comparatively permanent coloration appears.

When the amount of vanadium in the rock is known a correction can be applied on the assumption that practically all the vanadium is here collected, a point that needs further investigation. Various authors assert its precipitability with alumina and iron by ammonia and ammonium-acetate, though Carnot $^{1}$ states that repeated precipitation by ammonia, ammonium carbonate, or ammonium sulphydrate, separates it from iron. The writer's experience with ores very rich in vanadium shows that precipitation along with iron and aluminum is only partial. Ridsdale ${ }^{z}$ has determined its precipitability with various metals and gives numerous figures which show an approximation to 90 per cent thus thrown down under the conditions prevailing in analysis of iron slags, the remainder passing into the filtrates and appearing in small part with the lime and to a greater extent with the magnesium phosphate. For all practical purposes it is probably safe to assume that the small amounts of vanadium met with in rocks are wholly in the alumina precipitate.

If the amount of vanadium in the rock is not known and greataccuracy is necessary, caution requires the determination of the total iron to be made either in a separate portion or after reprecipitation from the above solution, as follows: Fuse with sodium carbonate, extract with water, bring the insoluble residue into sulphuric solution, reduce and titrate as above directed. But unless a certain precaution is here observed an error greater than that which it is designed to avoid will be committed. Contrary to general belief the aqueous extract from the sodium-carbonate fusion carries a small but appreciable fraction of a per cent of iron, as the writer has repeatedly found by actual test. This iron is thrown out with the alumina (and silica, if present) by the usual methods of neutralizing the alkaline solution, and can be brought to light when the precipitate thus formed is treated with a fixed caustic alkali, or again fused with sodium carbonate and leached with water,

${ }^{1}$ Comptes Rendus, Vol. CIV; p. 1803, 1887; Zeitschr, für. anal. Chem., Vol. XXXII, p. 223, 1893.

2 Jour. Soc. Chem. Industry, Vol. VII, p. 73, 1888. 
when it remains wholly or in part undissolved. Hence it is necessary to collect this iron and add it to the main portion before titration.

DETERMINATION OF THE TRUE VALUE FOR FERRIC IRON.

Having in one way or another found the total iron in the rock, it remains to deduct an amount equivalent to the ferrous oxide the rock contains, and a further amount corresponding to the sulphides often present, in order to get what may pass for the true value for ferric iron. That this is often only an approximation appears from the difficulties due to the presence of vanadium and the generally indeterminable effect of sulphides on the ferrous oxide determination. (See pp. 94-96.)

\section{METHODS AIMING AT THE MORE OR LESS DIRECT ESTIMATION OF ALUMINUM.}

\section{AFTER FIRST REMOVING IRON AS SULPHIDE.}

Should it be desirable for any reason to effect an actual separation of aluminum, this may best be done, up to a certain point, after the bisulphate fusion (p. 56), by removal of the iron ${ }^{1}$ by ammonium sulphide in ammonium tartrate solution, evaporation of the filtrate, ignition of the residue with sodium carbonate and nitrate, and extraction with water, whereby titanium and zirconium are left on the filter as sodium salts while chromium and vanadium are carried into the filtrate as chromate and vanadate along with aluminum and phosphorus. The further separation of the two last from the chromium and vanadium is outlined under Phosphorus, p. 79. This is as far as the separation can well be carried, and the $\mathrm{Al}_{2} \mathrm{O}_{3}$ must still be found by subtracting the $\mathrm{P}_{2} \mathrm{O}_{5}$ from the combined weights of the $\mathrm{Al}_{2} \mathrm{O}_{3}$ and $\mathrm{P}_{2} \mathrm{O}_{5}$. The possibility of loss of some $\mathrm{P}_{2} \mathrm{O}_{5}$ by volatilization ${ }^{2}$ during the bisulphate fusion must be borne in mind here, for if it takes place the final weight of $\mathrm{Al}_{2} \mathrm{O}_{3}+\mathrm{P}_{2} \mathrm{O}_{5}$ will not contain all the $\mathrm{P}_{2} \mathrm{O}_{5}$.

Some writers recommend dissolving the ignited alumina, iron oxide, etc., in hydrochloric acid, but when the precipitate has been heated over the blast, as it should be, this is very ineffective.

BY EXTRACTION With a FiXed CAUSTIC ALKALI.

A favorite practice in some countries of Europe is to fuse the ignited precipitate containing $\mathrm{Al}_{2} \mathrm{O}_{3}, \mathrm{Fe}_{2} \mathrm{O}_{3}, \mathrm{TiO}_{2}, \mathrm{P}_{2} \mathrm{O}_{5}$, etc. - or that of the $\mathrm{Al}_{2} \mathrm{O}_{3}, \mathrm{TiO}_{2}, \mathrm{P}_{2} \mathrm{O}_{6}$, etc., after separation of iron by ammonium sulphide in tartrate solution-with sodium hydroxide in a silver crucible, or to

1 This being first reduced to the ferrous condition by hydrogen sulphide in acid solution in order to obviate the possibility of precipitating some titanium, which otherwise is likely to happen. (Cathrein, Zeitschr. für Kryst., Vol. VI, p. 246, 1882, and Vol. VII, p. 250, 1883.)

$2 \mathrm{H}$. Rose speaks of such loss when volatilizing sulphuric acid in presence of phosphoric acid. (Handb. f. quant. Anal., Finkener edition, Vol.II, p. 575, and elsewhere.) 
boil the freshly precipitated mixture.with a solution of the alkali, on the assumption that the titanium oxide is hereby rendered wholly insoluble and thus separated from the alumina. This, however, is in part an error long since pointed out by Gooch, ${ }^{1}$ who showed that pure titanic oxide is markedly soluble under both conditions of treatment. Experiments very recently made by the writer to test the extent of this error brought out the following interesting results.

When 0.045 gram of titanic oxide was fused by itself with sodium hydroxide, the clear aqueous extract of the fusion held $0.0031 \mathrm{TiO}_{2}$, or about 7 per cent, determined colorimetrically. When freshly precipitated and boiled with the alkali the solubility was less. When fused with sodium carbonate but an infinitesimal trace was dissolved, which required strong concentration for its detection. When mixed with a large excess of alumina and fused with the caustic alkali, the solubility was still very marked, though less than when alumina was absent. With a large excess of ferric oxide, with or without alumina, no titanium could be detected in the unconcentrated filtrate.

It thus appears that fusion with caustic alkali after first removing iron involves an error in the gravimetric determination of both aluminum and titanium which does not appear if the iron has not been removed.

DIRECT PRECIPITATION OF ALUMINA.

A recent and promising method for the "direct determination of alumina in presence of iron, manganese, calcium, and magnesium" is that of Hess and Campbell, ${ }^{2}$ but, as with the methods just considered, it involves finally weighing aluminum and phosphorus together, and the behavior of titanium has not been investigated. For this latter reason the details of the method will not be given. Suffice it to say that precipitation of the aluminum and phosphorus is made by phenylhydrazine, after first neutralizing the (preferably chloride) solution by ammonia and reducing iron by a saturated solution of ammonium bisulphite. Phenylhydrazine "precipitates aluminum from its solutions quantitatively as the hydroxide without a trace of the precipitate being redissolved in excess of the precipitant."

IX. MANGANESE, NICKEL, COBALT, COPPER, ZINC.

Ammonia is added to the flask containing manganese, the earths, etc. (p. 56), and hydrogen sulphide gas is introduced, whereby manganese, nickel, cobalt, copper, zinc, and a small part of the platinum from the dish are precipitated. The flask is set aside, corked, for at least twelve hours, and preferably twenty-four, or even longer; the pre-

1 Proc. Am. Acad. Arts and Sci., Vol. XII, p. 436, 1885; Bull. U. S. Geol. Survey No. 27, pp. 16 and 17.

2Jour. Am. Chem. Soc., Vol. XXI, p. 776, 1899; Chemical News, Vol. LXXXI, p. 158, 1900. 
cipitate, collected and washed on a small filter with water containing ammonium chloride and sulphide, is extracted by hydrogen-sulphide water acidified with one-fifth its volume of hydrochloric acid (sp. gr. 1.11), manganese and zinc, if present, going into solution.

\section{MANGANESE AND ZINC.}

The filtrate is evaporated to dryness, ammonium salts are destroyed by evaporation with a few drops of sodium-carbonate solution, hydrochloric and a drop of sulphurous acids are added to decompose excess of carbonate and to dissolve precipitated manganese, and the latter is reprecipitated at boiling heat by sodium carbonate after evaporation of the hydrochloric acid. If zinc is present, it can be separated from the manganese after weighing. For the small quantities of manganese usually found the sodium-carbonate method of precipitation is to be preferred to that by bromine or sodium phosphate, as equally accurate and a great time saver.

The precipitation of manganese in alkaline solution by hydrogen peroxide, as proposed by Jannasch and Cloedt, ${ }^{1}$ a method which appeared to be simple and accurate, besides affording a separation from zinc, has been shown by Friedheim and $\mathrm{Brühl}^{2}$ to be valueless, as also other separation methods of Jannasch based on the use of hydrogen peroxide.

The employment of ammonium sulphide instead of bromine for the separation of manganese from the alkaline earths and magnesia has the advantage that, by a single operation, nickel, copper, and zinc are likewise removed if present. There need be no fear of overlooking nickel or copper, for under the conditions of the precipitation they are not held in solution. Now and then a trace of alumina may be found in the precipitate, and magnesia, too, would contaminate it if ammonium salts were not present in sufficient quantity. Regard must therefore be had to these possibilities, and also to the rather remote possibility of the presence of rare earths which were not thrown out by the basic acetate precipitation (see footnote, p. 55).

\section{NICKEL, COBALT, COPPER.}

The paper containing these is incinerated in porcelain, dissolved in a few drops of aqua regia, evaporated with hydrochloric acid, the copper and platinum thrown out warm by hydrogen sulphide, and nickel and cobalt thrown down from the ammoniacal filtrate by hydrogen sulphide. This is then rendered faintly acid by acetic acid and allowed to stand. The sulphide of nickel is simply burned and weighed as 
oxide, its weight being always very small, and is then tested for cobalt in the borax bead.

It is somewhat unsafe to consider traces of copper found at this stage to belong to the rock if the evaporations have been conducted, as is usually the case, on a copper water bath, or if water has been used which has been boiled in a copper kettle, even if tinned inside. Therefore, and because of its contamination by a little platinum, it is better to determine copper in a separate portion if its presence is indicated with certainty. (See p. 54.)

\section{CALCIUM AND STRONTIUM (BARIUM).}

SEPARATION FROM MAGNESIUM.

Precipitation and ignition of the oxalates together.-The platinum derived from the dish in the silica evaporation, except for the small portion precipitated with the manganese sulphide; is now wholly in the filtrate from the latter. Its separation at this or any other stage is quite unnecessary; nor is the removal of ammonium chloride usually demanded, since there is no undue amount present in most cases, the first precipitation of alumina, etc., having been by sodium acetate. ${ }^{1}$ Therefore, without destroying ammonium sulphide the calcium and strontium are thrown out by ammonium oxalate at boiling heat, the precipitate, often darkened by deposited platinum sulphide, is ignited and redissolved in hydrochloric acid, boiled with ammonia to throw out traces of alumina sometimes present and reprecipitated as before, but in a small bulk of solution. It is weighed as oxide, transferred to a small flask of $20 \mathrm{~cm}^{3}$ capacity, dissolved in nitric acid, evaporated to dryness at 150 to $160^{\circ} \mathrm{C}$., and the separation of strontium from calcium effected by ether-alcohol ${ }^{2}$ as described below.

The weight of .strontia found deducted from that of the two oxides gives that of the lime.

Necessity for two precipitations by ammonium oxalate.-It may be said with regard to the separation of calcium from magnesium that two precipitations by ammonium oxalate are essential to the attainment of correct results, not only for the complete removal of magnesium but of sodium as well, the retention of compounds of the latter element by calcium oxalate being now generally known. For the treatment of the filtrates, see Magnesium, p. 64.

\footnotetext{
${ }^{1}$ If two or three precipitations by ammonia alone are depended on, the second and third filtrates are evaporated rapidly to dryness and the ammonium salts removed by ignition.

${ }^{2}$ See Fresenius: Zeitschr. für anal. Chemie, Vol. XXXII, pp. 189, 312, 1893, for the. latest improvements in this method.
} 
SEPARATION OF STRONTIUM (BARIUM) FROM CALCIUM BY ETHER-ALCOHOL.

The thoroughly dried nitrates are treated with as little (rarely over $2 \mathrm{~cm}^{3}$ ) of a mixture in equal parts of absolute alcohol and ether as may be needed to dissolve the calcium salt, solution being hastened by occasional gentle agitation. After standing over night in a corked flask the insoluble matter is collected on the smallest possible filter and washed with more of the above mixture of alcohol and ether. After drying, a few cubic centimeters of hot water are passed through the filter, on which may remain a few tenths of a milligram of residue which does not usually contain any lime or other alkaline earth and - whose weight is therefore to be deducted from that of the lime, unless it can be shown that it is derived from the glass of the little flask in which the nitrates of calcium and strontium were evaporated. To the solution of strontium nitrate in a small beaker sulphuric acid and then alcohol is added, whereby the strontium is precipitated as sulphate, in which form it is weighed and then tested spectroscopically as to freedom from calcium and barium.

Because of the slight solubility of strontium nitrate in amyl alcohol the method of Browning ${ }^{1}$ does not appear to be adapted to the separation from calcium of the small amounts of strontium met with in rocks, though with barium the case is different, since its nitrate according to Browning is insoluble in absolute amyl alcohol.

\section{BEHAVIOR OF BARIUM.}

Barium will, after two ammonium oxalate precipitations, never be found with the ignited calcium and strontium in more than spectroscopic traces, unless originally present in excess of 3 or 4 milligrams, and very often only when in considerable excess. ${ }^{2}$ If present with them, however, it will be separated with the strontium by. ether-alcohol or amyl alcohol, and these two must then be treated by the ammoniumchromate method, given below, in order to arrive at the strontium. The barium is best estimated in a separate portion. (See Barium, p. 73.)

\section{SEPARATION OF BARIUM FROM STRONTIUM.}

Fresenius has show $\mathrm{n}^{3}$ in what manner only a correct separation of barium and strontium can be made by the ammonium-chromate method, involving double precipitation when the amounts are at all large. This procedure is here given for the amounts used by him, but a single precipitation will suffice for the small amounts met with in rock analysis.

${ }^{1}$ Am. Jour. Sci., 3d series, Vol. XLIII, pp. 50, 314, 1892.

2W. F. Hillebrand: Jour. Am. Chem. Soc., Vol. XVI, p. 83, 1894; Chemical News, Vol. LXIX, p. 147, 1894.

${ }^{3}$ Zeitschr. für aual. Chemie, Vol. XXIX, p. $428 ; 1890$. 
The volumes of solutions used should be largely reduced and the operations otherwise shortened.

The chlorides corresponding to 0.2774 gram $\mathrm{BaO}$ and 0.4864 gram $\mathrm{SrO}$ were dissolved in $300 \mathrm{~cm}{ }^{3}$ of water with addition of 6 drops of acetic acid (1.065 sp. gr.). To the hot solution was added an excess $\left(10 \mathrm{~cm}^{3}\right)$ of ammonium chromate solution $\left(1 \mathrm{~cm} .{ }^{3}=0.1 \mathrm{gr}\right.$. neutral chromate). After settling and cooling for an hour the precipitate was washed, mainly by decantation, with water holding ammonium chromate till the filtrate gave no precipitate with ammonia and ammonium carbonate $\left(100 \mathrm{~cm}^{3}\right.$ used). The washing was continued with warm water till silver nitrate gave but a very slight reddish coloration (110 $\mathrm{cm} .{ }^{3}$ ). The precipitate was then washed into the precipitating dish, the filter rinsed with warm dilute nitric acid (1.2 sp. gr.), and more nitric acid $\left(2 \mathrm{~cm} .{ }^{3}\right.$ in all $)$ added to the dish. The solution having been diluted to $200 \mathrm{~cm} .{ }^{3}$ and heated, $5 \mathrm{~cm}^{3}$ of ammonium acetate solution $\left(1 \mathrm{~cm} .^{3}=0.31 \mathrm{gr}\right.$. ammonium acetate $)$ was very gradually added, and then ammonium chromate till the odor of acetic acid had wholly disappeared $\left(10 \mathrm{~cm}^{3}\right)$. After one hour the supernatant liquid was passed through the filter and the precipitate digested with hot water, which was then cooled; thereupon the precipitate itself was brought on the filter and washed with cold water till silver nitrate gave a scarcely perceptible reaction. The strontium was thrown down from the filtrate by ammonia and ammonium carbonate, after concentration in presence of a little nitric acid, and weighed as carbonate; or the carbonate can be redissolved, precipitated by sulphuric acid and alcohol, and weighed as sulphate. The barium is weighed as chromate after ignition, the filter being burned separately.

\section{MAGNESIUM.}

\section{PRECIPITATION.}

The first precipitation of magnesium is made without special precautions in the filtrate from the first calcium oxalate separation (p. 62) by sodium-ammonium-hydrogen phosphate (microcosmic salt) ${ }^{1}$ in indefinite decided excess and without the great excess of ammonia usually prescribed. It is not necessary to first remove ammoniacal salts unless very little magnesium is present, and then only in order to hasten precipitation. Neubauer ${ }^{2}$ has shown that precipitation is complete even in presence of large quantities of salts of ammonium, including the oxalate. He has, however, also shown that the composition of the precipitate is largely affected by ammonium salts, and also by the way in which the

\footnotetext{
1 The objection that has been made by one writer to the use of this salt instead of disodium-hydrogen phosphate is, so far as our experience tenches, entirely groundless.

2 Zeitschr. für angew. Chemie, 1896, p. 435.
} 
precipitation is made. These points are only of importance when a single precipitation is to be made or in the final of two or more, as will be discussed later.

Platinum sulphide usually strongly contaminates the separated phosphate, but this matters not; as it remains on the filter when the phosphate is redissolved in hydrochloric acid, of which not more than the amount really needed should be used. The solution thus obtained is united with that of the residue from evaporation and ignition of the second filtrate from calcium oxalate, and is diluted if necessary. A few drops of sodium-ammonium phosphate solution are now added, and ammonia in slight excess, with constant stirring till the crystalline precipitate has well formed. The large excess of ammonia of 0.96 specific gravity (one-third the original volume) usually prescribed has been shown by Gooch and Austin ${ }^{1}$ to be quite unnecessary, in fact, disadvantageous.

Neubauer in the above-cited paper has shown, and Gooch and Austin (loc. cit.) have confirmed his statements, that it is only by working under these conditions-absence of any large excess of precipitant, of ammoniacal salts, and of ammonia-that a precipitate of normal composition is obtainable. It usually differs from the normal in contain-

- ing relatively more ammonium and less magnesium-for instance, an admixture of such a molecule as $\mathrm{Mg}\left(\mathrm{NH}_{4}\right)_{4}\left(\mathrm{PO}_{4}\right)_{2}$-the result being that when ignited in the ordinary way too much magnesium is found, because of formation of some metaphosphate. To obviate this error Neubauer considers it absolutely necessary to blast the precipitate for half an hour, and then to repeat the blasting for a second half hour to see if a constant weight has been reached. The phosphate is then entirely pyrophosphate, which is quite unaffected by further blasting. The intense heat has caused a decomposition of the metaphosphate with volatilization of $\mathrm{P}_{2} \mathrm{O}_{5}$, as follows: $2 \mathrm{Mg}\left(\mathrm{PO}_{3}\right)_{2}=\mathrm{Mg}_{2} \mathrm{P}_{2} \mathrm{O}_{7}+\mathrm{P}_{2} \mathrm{O}_{5}$. Neubauer worked with the usual excess of ammonia, and it remains to be seen whether by precipitating and working according to Gooch and Austin the composition of the precipitate is always close enough to the ideal $\mathrm{MgNH}_{4} \mathrm{PO}_{4}$ to obviate the necessity for blasting.

From the labor's of Neubauer and of Gooch and Austin it is clear that the common way of adding the phosphate precipitant to the ammoniacal solution of the magnesium salt is not calculated to produce a precipitate of normal composition. The precipitant should be added to the acid solution of the magnesium, and ammonia should then be added in slight excess.

Gooch and Austin call attention to a modification proposed some years ago by Wolcott Gibbs, ${ }^{2}$ whereby the phosphorus and magnesium

${ }^{1}$ Am. Jour. Sci., 4th series, Vol. VII, p. 187, 1899; Chemical News, Vol. LXXIX, pp. 233, 244, 255, 1899; Zeitschr. für anorg. Chemie, Vol. XX, p. 121.,1899.

2 Am. Jour. Sci., 3d series, V.ọl. V, p. 114, 1873.

Bull. 176--5 
salts are first boiled together in neutral solution for a few minutes and to the cooled solution ammonia is added. The results are said to be remarkably exact.

\section{METHODS OF COLLECTING AND IGNITING THE PRECIPITATE.}

If the blast has not to be employed, the weight of the pyrophosphate can doubtless be most accurately arrived at by collecting and igniting the precipitate in a Gooch crucible, provided the asbestos felt is well constructed and not of the serpentine variety so largely on the market.

Neubauer ignites slowly in platinum after drying, without removing from the paper, applying the blast only when the carbon has been wholly burned off. ${ }^{1}$

Almost as exact are the two modifications of the method in use for phosphate analyses at the agricultural experiment stations at Danzig and Lisbon, described by Schmoeger ${ }^{2}$ and Mastbaum. ${ }^{3}$ According to the former the precipitate after drying is detached from the paper and placed in a platinum crucible, followed by the folded filter. To the covered crucible the full flame of a burner is applied, and after a short time the burning off of the carbon is accomplished with the crucible open. A short blasting follows. In a number of experiments on quantities ranging from 0.06 to 0.28 gram of pyrophosphate the results were, with a few exceptions, naturally lower than those obtained on duplicates by the ordinary method of igniting, but only by 0.0013 gram in maximum.

Mastbaum, to shorten time, applies the full flame to the moist precipitate wrapped in its filter. Later, when most of the carbon is burned off, he moistens the residue with two or three drops of strong nitric acid, evaporates this carefully, heats with full burner for a few minutes, then blasts for half a minute. He describes the results as irreproachable. In this laboratory only the Mastbaum modification has been tried, and it certainly seems to be satisfactory when the extreme of accuracy is not required.

At one time the procedure first recommended by Ulbricht, later by Broockmann, and also by L. L. de Koninck, was used. It consists in dissolving the ammonium-magnesium phosphate off the filter with nitric acid, collecting the filtrate in a weighed crucible, evaporating the contents to dryness, ${ }^{4}$ and subsequently igniting, the product being presumably pyrophosphate. But it was soon observed that the ignited salt, especially when large in amount, does not always dissolve com-

\footnotetext{
1 Zeitschr. für anal. Chemie, Vol. XXXIII, p. 362, 1894.

2 Ibid., Vol. XXXVII, p. 308; 1898.

3Ibid., p. 581, 1898.

${ }^{4} \mathrm{~A}$ pink color of varying intensity almost invariably becomes apparent as the mass approaches dryness, a most delicate test for the traces of manganese which always escape precipitation by ammonium sulphide or bromine.
} 
pletely in hydrochloric acid, but that sometimes a white residue is left in light lumps which appears to be quite insoluble in acids. This residue contains no silica, but only the constituents of a magnesian phosphate, and it may be a peculiar metaphosphate. Whether its appearance is due to an abnormal composition of the original magnesian precipitate or to conceivable change during evaporation in the crucible with nitric acid remains to be determined. Until this is done the employment of this method of igniting is not to be recommended.

\section{CONTAMINATION BY AND REMOVAL OF BARIUM AND CALCIUM.}

Barium phosphate will not contaminate the second magnesian precipitate unless there are notable amounts of barium in the rock, in which case it must be removed by sulphuric acid prior to the final. precipitation of the magnesium. Calcium, however, is probably never absent, and has to be estimated and allowed for as follows:

To the ignited pyrophosphate, dissolved in but slight excess of hydrochloric acid, is added ammonia to alkalinity, and then acetic acid, drop by drop, till the solution, which should not be hot, clears. It now and then happens that a little flocculent matter fails to dissolve. This is to be removed, ignited, and subtracted from the original weight. It is likely to consist, in great part or wholly, of phosphates of iron or manganese, or both, and shows often a reddish color on ignition. If an excess of acetic acid has been used, this is cautiously removed by ammonia. Then a drop or two of solution of ammonium oxalate is added, and the small beaker: is set aside for twelve hours if necessary. Almost invariably a small precipitate soon shows itself, which if finegrained and nonadherent to the glass may be regarded as pure calcium oxalate; otherwise it contains, or may largely consist of, magnesium oxalate. It is in that case to be collected, ignited, redissolved, and reprecipitated. Its final weight, averaging perhaps one-half milligram, is to be added to that of the lime already found, and subtracted as tricalcium phosphate from that of the magnesium pyrophosphate in order to arrive at the true figure for magnesia. This separation, to be satisfactory, requires great care.

\section{TITANIUM.}

COLORIMETRIC ESTIMATION WITH HYDROGEN PEROXIDE (WELLER'S METHOD). ${ }^{1}$

The method consists in comparing the color of a known bulk of solution to be tested with that of a standard solution of titanium sulphate, both having been fully oxidized by hydrogen peroxide. The strength 
of the peroxide should be approximately measured by titration with permanganate on opening a fresh bottle, and again after a few weeks, otherwise very serious error may arise through its deterioration.

Mere traces of hydrofluoric acid, either in the peroxide or the titanium solution, render this method inexact, ${ }^{i}$ hence care should be exercised as to the character of the peroxide, which, as sold in the market, often contains fluorine.

Dunnington $^{2}$ has pointed out the necessity for the presence of at least 5 per cent of sulphuric acid in solutions which are to be thus tested for titanium, in order, as he concludes, to prevent partial reversion to metatitanic acid, which does not give a color with hydrogen peroxide. The standard solution of titanium sulphate, holding conveniently about 1 centigram $\mathrm{TiO}_{2}$ in $10 \mathrm{~cm}{ }^{3}$, equivalent to 1 per cent of $\mathrm{TiO}_{2}$ in 1 gram of rock, contains, therefore, 5 per cent or more of sulphuric acid. Of this, $10 \mathrm{~cm} .{ }^{3}$ are mixed with a sufficiency of hydrogen peroxide $\left(2 \mathrm{~cm} .^{3}\right.$ of most commercial brands is ample) and diluted to $100 \mathrm{~cm} .^{3}$ in a measuring flask.

Titanium can be estimated, as a rule, most conveniently in the solution which has served for the titration of total iron (p. 57). This, having been evaporated, if necessary, to less than $100 \mathrm{~cm}^{3}$, is to be fully oxidized with hydrogen peroxide, and if the color is less intense than that of the standard, is made up to $100 \mathrm{~cm} .{ }^{3}$ with dilute sulphuric acid in a measuring flask, and mixed; otherwise, in a flask of sufficient size to insure that its color shall be less intense. One of the rectangular glasses described below being filled with the solution to be tested, 10 $\mathrm{cm} .{ }^{3}$ of the diluted standard are run into the other from a burette, and water is added from a second burette until there is no distinction as to color. A second and a third portion of the standard can be run in and diluted and the mean of several determinations struck, when a simple calculation gives the percentage of $\mathrm{TiO}_{2}$ in the rock.

If the convenient but expensive Soleil-Duboscq colorimeter is used, or the simple Nessler tubes, it is of course unnecessary to dilute the rock solution to the extent above required, should it be stronger than the standard. Experience has shown, however, that differences can not be sharply estimated in strongly colored solutions, and that the results are much more satisfactory when the color intensity.is not much, if any, greater than that given by a standard of the above concentration. For the percentages of titanium found in rocks, clays, and soils, usually under 1 per cent, but rising to 2 or even 3 per cent or more . occasionally, the colorimeter method gives results which are fully equal to those of the best gravimetric method, besides being a great time

\footnotetext{
'Hillebrand: Jour. Am. Chem. Soc., Vol. XVII, p. 718, 1895; Chemical News, Vol. LXXII, p. 158, 1895; Bull. U.S. Geol.Survey No.167, p. 56.

2 Jour. Am. Chem. Soc., Vol. XIII, p. 210, 1891.
} 
saver. The error introduced by iron, in consequence of the yellowish color of its sulphate solution, is practically negligible unless its percentage is very high; then either the iron must be removed prior to making the color test, or correction should be applied for known amounts of ferric sulphate in solutions of the requisite dilution.

The exact correction to be applied in such cases is difficult of determination because of the impossibility of matching the colors of titanium peroxide solutions with those of ferric sulphate; but tests made go to show that the coloring effect of 0.1 gram of $\mathrm{Fe}_{2} \mathrm{O}_{3}$ in $100 \mathrm{~cm} .{ }^{3} 5$ per cent sulphuric-acid solution is about equal to 0.2 milligram of $\mathrm{TiO}_{2}$ in $100 \mathrm{~cm} .^{3}$ when oxidized by hydrogen peroxide. This amounts to a correction of only 0.02 per cent on 1 gram of rock containing the unusual amounts of 10 per cent $\mathrm{Fe}_{2} \mathrm{O}_{3}$. It will be more satisfactory, when much iron is present, to remove this as described on page 71 and to colorimetrically estimate the titanium thus freed from iron.

ALTERNATIVE MODE OF PREPARING THE TEST SOLUTION.

As said above (pp. 57 and 68 ), the solution that has been used for volumetric estimation of total iron can most conveniently be used for the colorimetric determination of titanium, but if desired this can, of course, be made on some other portion of rock powder. At one time it was the practice in this laboratory to combine it with the determination of barium, as described in Bulletin 148 of the United States Geological Survey, by decomposing the powder by sulphuric and hydrofluoric acids, ${ }^{1}$ expelling the latter by repeated evaporations with sulphuric acid, taking up with dilute sulphuric acid, ${ }^{2}$ filtering from barium sulphate, etc., and estimating the titanium colorimetrically in the filtrate. The expulsion of fluorine must be thorough, or else the titanium result will be low, as already ${ }^{\circ}$ stated (p. 68), ${ }^{-}$and it is not always easy to effect this complete removal, though the time required to do so seems to be in no slight degree dependent on the nature of the fluorides to be decomposed. Long after every trace of fluorine seems to be gone, the formation of a crust on the evaporating solution sometimes allows an accumulation of enough hydrofluoric-acid gas to become plainly manifest to the smell on breaking the crust.

\footnotetext{
It is to be borne in mind that evaporation with hydrofluoric acid alone results in loss of titanium by volatilization, but that there is no loss if excess of sulphuric acid is also present.

2 With acid rocks solution is very complete, and it can be made nearly so with the most basic by transference to a small beaker and gentle boiling. The residue thus obtained may contain, besides barium sulphate, a little calcium sulphate, zircon, andalusite, topaz, and possibly a trace of titanium ' in some form. It is therefore to be thoroughly fused with sodium carbonate, leached with water, fused with potassium bisulphate, dissolved in dilute sulphuric acid, filtered, and the filtrate arded to the main one. The insoluble matter will now be chiefly barium sulphate, for the further treatment of which see page 74 .
} 
THE COLORIMETRIC APPARATUS AND ITS USE.

The glasses $\mathrm{G}$ (fig. 12) may be of square or rectangular section, 8 to . $12 \mathrm{~cm}$. high and 3 to $3 \frac{1}{2} \mathrm{~cm}$. inside measurement between those sides through which the liquid is to be observed. ${ }^{1}$ These sides should, of course, be exactly parallel; the others need not be, but should be blackened externally. In order to further exclude the effect of side
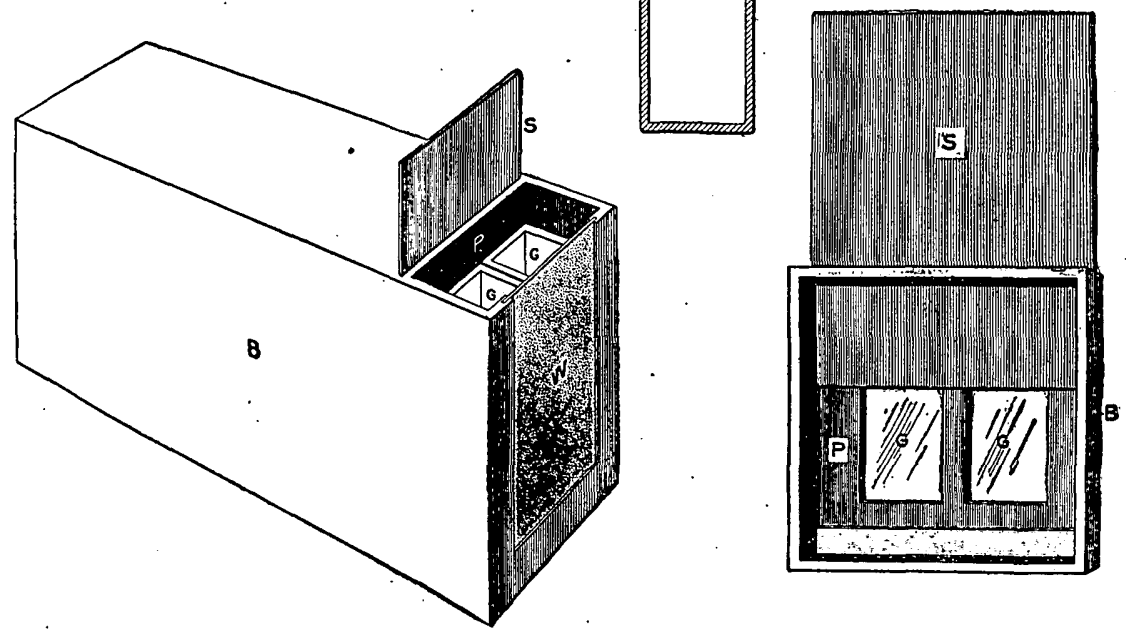

FIG. 12.-Apparatus for colorimetric determinations, in different aspects. G, one of two glasses of square or reetangular section, 8 to $12 \mathrm{~cm}$. high and 3 to $3 \frac{1}{2} \mathrm{~cm}$. inside measurement between those sides through which the liquid is to be observed. The other sides are blackened on the outside. $B$, rectangular box about $35 \mathrm{~cm}$. long and $12 \mathrm{~cm}$. square, stained black inside and out, one end closed by a ground-glass window, $W$, the other open, and a portion of the top removed. P, blackened partition, with openings corresponding to the interior dimensions of the glasses when in position. S, blackened cardboard shutter sliding stiffly up and down between partition and glasses, so as to shut off all light above the lowest surface of the liquid in the glasses.

light in this and other similar methods (chromium, for instance, p. 80), it is very convenient to have a simple light box ( $B$, fig. 12$)$ that can be easily held in one hand, about $35 \mathrm{~cm}$. long and $12 \mathrm{~cm}$. square, stained black inside and out and with one end closed by a piece of ground glass $\mathrm{W}$, the other open. For a space equal to the width of the glasses the

1 The allowable error in distance between the corresponding pairs of sides of the two glasses should not in any case exceed 1 per cent. Unfortunately there seems to be a disinclination or inability on the part of dealers in this country to furnish glasses fulfilling this requirement, and held together by a durable cement which shall be proof against dilute sulphuric acid. Canada balsam answers well for a time, but sooner or later it cracks, leaks then appear, and the sides soon drop off. It is, however, but a simple matter to cement them on again.

A pair of entirely satisfactory glasses can be made from a couple of square or rectangular 3 to 4 ounce bottles by cutting off one pair of sides from each and grinding down till the calipers show that agreement is perfect. The tops are then to be sawed off and pieces of plate glass cemented on the sides. 
cover is removed at the top next the glass end to permit of the insertion of the glasses side by side in such a way that no light shall penetrate around their sides or between them. Immediately back of the glasses is a partition $\mathrm{P}$, with openings of appropriate size cut in it. A stiffly sliding black cardboard shutter $\mathrm{S}$ is movable up and down immediately back of the partition, so that all light can be cut off except that which comes through the liquid.

Precautions of this kind are necessary if accurate results are to be counted on. Except for mere traces, this combination of glasses and darkened box insures greater accuracy and rapidity of work than Nessler tubes, and is preferable likewise, so far as the writer's experience goes, to expensive instruments like the colorimeter of Soleil-Duboscq, etc. In making the color comparisons the box is best held close to a window, so as to get a full, strong light. Daylight is far preferable to artificial light.

\section{GOOCH'S GRAVIMETRIC METHOD.}

When titanium is present in excess of 4 to 5 per cent and whenever for any reason it is desired to employ a gravimetric method, among the few that have been thoroughly tested that of Dr. Gooch ${ }^{1}$ is unequaled. With one or two minor modifications introduced by $\mathrm{Dr}$. T. M. Chatard, ${ }^{2}$ it is as follows:

Any solution of the rock freed from silica can be used, and the first step is to remove the iron. This is best done, after adding tartaric acid and reducing the iron by means of hydrogen sulphide to the ferrous condition, by rendering the solution ammoniacal and introducing more hydrogen sulphide. If the iron is not thus reduced before precipitation, titanium will be in part thrown down also. ${ }^{3}$ The amount of tartaric acid is to be gauged according to the combined weights of the oxides to be held by it in solution, and three times this weight is ample. After removing the iron sulphide by filtration-little washing suffices, because of the relatively small amount of titanium commonly present-the tartaric acid is destroyed as follows:

Potassium permanganate to the extent of two and one-half times the weight of the tartaric acid used is made into a strong solution, and to the ammoniacal filtrate from the iron sulphide enough sulphuric acid is introduced to leave some excess after all the permanganate has been reduced. After expulsion of hydrogen sulphide by boiling the permanganate is added gradually to the hot solution contained in a large beaker or flask. A vigorous reaction ensues. When a permanent brown precipitate of manganic hydrate appears the tartaric acid has

1 Proc. Am. Acad. Arts Sci., n. s., Vol. XII, p. 435; Bull. U.S. Geol. Survey, No. 27, p. 16, 1886; Chemical News, Vol. LII, pp. 55 and 68,1885 .

${ }^{2}$ Am. Chem. Jour., Vol. XIII, p. 106, 1891; Bull. U. S. Geol. Survey, No. 78, p. 87; Chemical News, Vol. LXIII, p. 267, 1891.

${ }^{3}$ Cathrein: Zeitschr., für Kryst., Vol. VI, p. 243, 1882; Vol. VII, p. 250, 1883. 
been fully broken up, and the precipitated manganese is to be redissolved by a few drops of ammonium bisulphite or of sulphurous acid solution.

Ammonia is then added in slight excess, followed at once by acetic acid in considerable excess, and the boiling is continued for a few minutes. Thereby the titanium is freed from most of the alumina, and from lime and magnesia if they had not been earlier removed, also from most of the manganese introduced. The precipitate is filtered and washed with water containing acetic and sulphurous acids, then ignited, fused thoroughly with sodium carbonate, and leached with water to remove phosphoric acid and most of the remaining alumina. The residue is again ignited and fused with sodium carbonate. To the cooled melt in the crucible strong sulpuric acid is to be added, wherein it dissolves readily by aid of gentle heat. This solution is to be poured into a small volume of cold water and the platinum it contains precipitated by hydrogen sulphide at or near boiling temperature. After filtering and cooling, ammonia is added till the titanium is just precipitated, and a measured volume, containing a known weight of absolute sulphuric acid, is then added-just enough to redissolve the precipitate. The solution is then made up with acetic acid in such amount that the final bulk shall contain from 7 to 11 per cent of absolute acid, and then enough solid sodium acetate is stirred in to more than take up the sulphuric acid introduced. Upon rapidly bringing the liquid to ebullition the titanium is precipitated in flocculent and easily filterable condition, and the precipitation is complete after a minute's boiling; provided all the prescribed conditions have been followed and zirconium is absent.

The precipitate is washed first with acetic acid of 7 per cent strength and then with hot water. After 15 to 20 minutes' ignition over a good burner it is in condition for weighing and will lose no more weight over the blast lamp. For large amounts of titanium a repetition of the sodium carbonate fusion, etc., should be made. The actual carrying out of all these operations, when once the method is understood, requires much less time than the detailed description would indicate.

GOOCH'S METHOD NOT DIRECTLY APPLICABLE TO ROCKS CONTAINING ZIRCONIUM.

Prior to the adoption of the colorimetric method, Dr. Gooch's was invariably used in this laboratory. Occasional inability to secure clean and complete precipitation by it was experienced, especially with a certain series of rocks rather poor in titanium. Long research showed the difficulty to be due to the presence of zirconium, which acts as a marked preventive of the precipitation of titanium by boiling in an acetic acid solution under the conditions of the Gooch method.

The above rocks were found to contain up to 0.2 per cent of $\mathrm{ZrO}_{2}$, and this amount was able to prevent precipitation of 0.3 per cent of 
$\mathrm{TiO}_{2}$. The titanium which came down in excess of this amount did not settle out in flocculent condition, as happens when zirconium is not present, and it was difficult to filter. After the removal of the zirconium in the manner to be hereafter described (p. 75), however, no difficulty was experienced in precipitating all the titanium with the usual ease.

SUPERIORITY OF TFE COLORIMETRIC AND GOOCH METHODS OVER THE OLDER ONES.

In view of the good results obtainable by the colorimeter method in all cases and by the Gooch method in the absence of zirconium, it is incomprehensible that the old method of precipitation by many hours' boiling in a nearly neutral sulphate solution in presence of sulphurous acid should still find adherents in any part of the world.

Attention has been directed (p. 60) to the error resulting from attempting to separate aluminum from titanium by either fused or dissolved sodium hyảroxide.

\section{BASKERVILLE'S METHOD.}

Baskerville ${ }^{1}$ has proposed the separation of titanium from iron and aluminum by boiling the neutralized solution of the chlorides for a few minutes in presence of sulphurous acid. The test separations as given by him are sharp, and a single precipitation is said to suffice, the titanium being free from iron and easily filterable. This last statement and the ready precipitability are fully confirmed by the experiments of the writer on titaniferous iron ores, but, although the titanium is completely thrown out, it carries with it a little iron, for instance, about 0.25 per cent $\mathrm{Fe}_{2} \mathrm{O}_{3}$ with 8 to 10 per cent $\mathrm{TiO}_{2}$. Zirconium would probably be likewise precipitated (see p. 77) and phosphorus perhaps also, but this last point hais not been investigated, neither has the applicability of the method to aluminous rocks been tested.

\section{BARIUM (ZIRCONIUM, TOTAL SULPHUR).}

Reasons for estimating barium in a separate portion of rock powder.It has been said above (p. 63) that only in very exceptional cases will barium be found with the calcium and strontium after two, or possibly three, precipitations of the latter as oxalate, since it passes into the filtrates with the magnesium, whence it may be obtained as sulphate after removal of ammoniacal salts. Addition of some alcohol insures also the recovery of traces of strontium if the rocks are very rich in it. But it is unsafe to regard the amount thus separated from the imagnesium as representing the total amount of barium in the rock. It 
will almost always be found lower than the truth, probably for the reason that there are opportunities during the analysis for slight losses. It is best to estimate it in a separate 2-gram portion, which may also serve with advantage for the estimation of zirconium and total sulphur.

Modes of attack and subsequent treatment.-If zirconium and sulphur are not to be looked for, the simplest procedure is to decompose the powder by sulphuric and hydrofluoric acids (see p. 69, under Titanium), and to complete the purification of the barium sulphate thus obtained in the manner: described in the third paragraph below.

If zirconium and sulphur are both to be likewise determined, decomposition is effected by fusing over the Bunsen flame and then over the blast with sulphur-free sodium carbonate and insufficient niter to injure the crucible, first fitting the latter snugly into a hole in asbestos board (Lunge) to prevent access of sulphur from the gas flame. In case sulphur is not to be regarded, the niter and asbestos board are omitted. After thorough disintegration of the melt in water, to which a drop or two of methyl or ethyl alcohol has been added for the purpose of reducing ${ }_{3}$ manganese, the solution is filtered and the residue washed with a very dilute solution of sodium carbonate free from bicarbonate. This is to prevent turbid washings. A yellow color in the filtrate indicates chromium.

For the further treatment of the filtrate see Sulphur, p. 106, and Chromium (colorimetric method), p. 80.

The residue is dissolved in quite dilute warm sulphuric acid (stronger acid may be used if barium only is sought) and filtered through the original filter. This, with its contents, is ignited, evaporated with hydrofluoric and sulphuric acid, and taken up with hot dilute sulphuric acid. The filtrate, added to the former one, now contains all the zirconium (see pp. 75-76 for its further treatment). The residue contains all the barium, besides some of the strontium, and perhaps a good deal of calcium. It is fused with sodium carbonate, leached with water, the residue dissolved off the filter by a few drops of hydrochloric acid, from which solution the barium is thrown out by a large excess of sulphuric acid. A single solution of the barium sulphate in concentrated sulphuric acid and reprecipitation by water suffices to remove traces of calcium which might contaminate it if the rock was one rich in calcium, and even strontium is seldom retained by it in quantity sufficient to give concern. Should this 'be the case, however, which will occur' when the $\mathrm{SrO}$ and $\mathrm{BaO}$ are together in the rock in, roughly speaking, 0.2 and 0.4 per cent, respectively, the only satisfactory way is to convert the sulphates into chlorides and to apply to the mixture the ammonium-chromate method of separation (p. 63).

Barium and strontium sulphates can be brought into a condition for testing spectroscopically by reducing for a very few moments the whole or part of the precipitate on a platinum wire in the luminous 
tip of a Bunsen burner, and then moistening with hydrochloric acid. This should be known to everyone, but probably is not.

The procedure outlined in the foregoing paragraphs for the estimation of calcium, strontium, and barium in silicate rocks is the one which long experience has shown to be best adapted for securing the most satisfactory results with a minimum expenditure of time. ${ }^{1}$ Even where no attempt is made to separate contaminating traces of $\mathrm{SrO}$ and $\mathrm{BaO}$ one from the other, the error is usually of no great consequence, for an absolute error of 25 per cent, even, in a substance constituting only one or two tenths per cent of a rock is ordinarily of small moment compared with the ability to certify to its presence with approximate correctness.

With such imall amounts of barium as are usually found in rocks it is doubtful if Mar's ${ }^{2}$ method for the separation of barium from calcium and magnesium, by the solvent action of concentrated hydrochloric acid mixed with 10 per cent of ether on the chlorides, could be conveniently applied here, although for larger amounts the method would seem to be accurate and easily executed. Moreover, it would probably not entirely remove contaminating strontium, and hence offers no advantage.

\section{ZIRCONIUM.}

This element is rarely looked for by chemists, though shown by the microscope to be one of the most constant rock constituents, usually in the form of zircon, in which occurrence its amount can be approximately judged of and a chemical test rendered almost unnecessary; but sometimes it occurs in other minerals, and is then unrecognizable under the microscope. It may rarely be present up to a few tenths of 1 per cent of the rock.

\section{AUTHOR'S METHOD.}

For its detection and estimation in such cases, or whenever a search for it seems called for, the following procedure, based on a method by G. H. Bailey, ${ }^{3}$ has been devised, which serves, when carried out with care, to detect with certainty the merest trace- 0.02 per cent, for instance-in $1 \mathrm{gram}$.

The preliminary treatment of the rock powder has been fully given under Barium (p. 74), where the separation from barium has been described and also the concentration of the zirconia in a small amount of very dilute sulphuric solution. This should probably not contain much above 1 per cent of sulphuric acid, though the actually permissible limit has not been established. To the solution, which

${ }^{1}$ For details consult W. F. Hillebrand: Jour. Am. Chem. Soc., Vol. XVI, p. 83, 1894; Chemical News, Vol. LXIX, p. 147, 1894.

2Am. Jour. Scl., 3d ser., Vol. XLIII, p. 521, 1892.

3 Jour. Chem. Soc., Vol. XLIX, pp, 149, 481, 1886. 
should be in a small flask, is now added hydrogen peroxide to oxidize the titanium, and then a few drops of a soluble orthophosphate solution. The flask is set aside in the cold for twenty-four to forty-eight hours. If the color bleaches after a time, more hydrogen peroxide should be added. Under these circumstances the zirconium is thrown out as phosphate and collects as a flocculent precipitate, which at this stage is not always pure. No matter how small or insignificant, it is collected on a filter, ignited, fused with sodium carbonate, leached with water, the filter again ignited, fused with very little acid potassium sulphate, brought into solution in hot water with a few drops of dilute sulphuric acid, poured into a flask of about $20 \mathrm{~cm} .^{3}$ capacity, a few drops of hydrogen peroxide and of sodium phosphate added, and the flask set aside. Titanium is now almost never present, and the zirconium appears after a time as a white flocculent precipitate, which can be collected and weighed as phosphate. For the small amounts usually met with it is safe to assume that it contains 50 per cent of $\mathrm{ZrO}_{2}(51.8$ by theory): If the amount is rather large, it may be fused with sodium carbonate, leached, ignited, fused with acid potassium sulphate, reprecipitated by ämmonia, and weighed as $\mathrm{ZrO}_{2}$. Certainty as to its identity can be had by again bringing it into solution, precipitating by ammonia, dissolving in hydrochloric acid, evaporating to a drop or two, and testing with turmeric paper or by a microchemical reaction. With the very smallest amounts no color can be obtained by this turmeric-paper test, which, however, responds readily to as little as 1 milligram of dioxide and with proper care for as small an amount as 0.3 milligram (Di. H. N. Stokes). No element other than thorium is ever likely to contaminate the zirconium thus precipitated.

In Bailey's experiments the precipitation was not made by addition of a phosphate, but is said to be due solely to the hydrogen peroxide, the precipitate being a hydrated peroxide, $\mathrm{Zr}_{2} \mathrm{O}_{5}$, or $\mathrm{ZrO}_{3} \cdot{ }^{1}$. My own efforts to secure a precipitate in acid solutions of zirconium sulphate by hydrogen peroxide alone were unsuccessful, perhaps for lack of a sufficiently strong peroxide. The ability to obtain the zirconium free from phosphoric acid would certainly be a great improvement on the method described above.

Were it not for the necessity of working in a weakly acid solution, the separation of zirconium could be made in the same portion in whick the titanium is colorimetrically determined.

\section{OTHER METHODS OF SEPARATING ZIRCONIUM.}

Streit and Franz ${ }^{2}$ claim to secure complete separation of titanium from iron and zirconium by boiling the neutralized solutions of the

${ }^{1}$ Bailey, Chemical News, Vol. LX, p. 6, 1889.

2 Jour. für prakt. Chemie, Vol. CVIII, p. 65, 1869. 
sulphates with a large excess (50 per cent) of acetic acid. The method has been from time to time recommended, but without any data showing its value. The single separation made by Streit and Franz was far from perfect.

Davis ${ }^{1}$ separated zirconium sharply from aluminum, but not from iron, by precipitation as an oxyiodate in a boiling neutralized solution of chlorides, but the method is hardly applicable for rock analysis.

Baskerville ${ }^{2}$ has proposed a method for the separation of zirconium from iron and aluminum similar to his method for the separation of titanium from those elements (p. 73). It is based on the precipitability of $\mathrm{ZrO}_{2}$ by boiling the neutralized chloride solution for two minutes in presence of sulphurous acid, and seems to be excellent. As titanium is always present and is presumably quantitatively thrown down also, the two would have to be separated by hydrogen peroxide. No tests as to the availability of the method for separating the small amounts met with in rock analysis have been made.

\section{RARE EARTHS OTHER THAN ZIRCONIA.}

For the few cases in which it may be necessary to look for rare earths other than zirconia, the following procedure is suggested as likely to prove satisfactory.

The rock powder is thoroughly decomposed by several partial evaporations with hydrofluoric acid, the fluorides of all earth metals except zirconium are collected on a platinum cone, washed with water acidulated by hydrofluoric acid, and the precipitate washed back into the dish or crucible and evaporated with enough sulphuric acid to expel all fluorine. The filter is burned and added. By careful heating the excess of sulphuric acid is removed and the sulphates are taken up by dilute hydrochloric acid. The rare earths, with perhaps some alumina, are then separated by ammonia, washed, redissolved in hydrochloric acid, and evaporated to dryness, then taken up with water and a drop of hydrochloric acid, and only enough ammonium acetate to neutralize the latter added, followed by oxalic acid (not ammonium oxalate, which would fail to precipitate thorium). In this way as little as 0.03 per cent of rare earths have been found when working on not more than 2 grams of materials.

This method eliminates at once most of the aluminum, all the iron, phosphorus, titanium, and zirconium, and has the further advantage of collecting with the earthy fluorides, as $\mathrm{UF}_{4}$, any uranous uranium that the rock might have held.

An alternative method would be to fuse with sodium carbonate, leach with water to get rid of phosphorus as far as possible, dissolve the

${ }^{1}$ Am. Chem. Jour., Vol. XI, p. 27, 1889.

2 Jour. Am. Chem. Soc., Vol. XVI, p. 475, 1894; Chemical News, Vol. LXX, p. 57, 1894. 
residue in hydrochloric acid, separate silica as usual, precipitate alumina, etc., by ammonia, dissolve the precipitate again in hydrochloric acid, evaporate, and proceed as by the former method, which in most cases would undoubtedly give better results than this one.

\section{PHOSPHORUS.}

It is sometimes possible to extract all phosphorus from a rock by simple digestion with nitric acid, but quite as often, if not oftener, this fails; hence the necessity for resorting to one of the longer methods of extraction detailed below.

\section{PROCEDURE WHEN MATERIAL IS AMPLE.}

When material is ample it is best to use one portion for phosphorus only and to proceed as follows:

Fuse with sodium carbonate, separate silica by a single evaporation with nitric acid, treat the ignited silica with hydrofluoric and a drop or two of sulphuric acids, evaporate to expel hydrofluoric acid, bring the small residue into solution by boiling with nitric acid and add it to the main portion, in which, after addition of considerable ammonium nitrate, precipitate the phosphorus by molybdate solution.

The turbidity often observed on dissolving the precipitated and washed phospho-molybdate in ammonia is due to a compound of phosphorus. If the addition of a small fragment of a crystal of citric or tartaric acid fails to dissolve it, this should always be re-fused with sodium carbonate, extracted with water and the filtrate otherwise treated as above, in order to secure the phosphorus in it.

According to Gooch and Austin, ${ }^{1}$ in order to secure a magnesiumammonium phosphate of normal composition, the procedure at this point should be as follows: To the phosphate solution, containing not more than 5 to 10 per cent of ammonium chloride and a slight excess of magnesia mixture, a little ammonia is added, and the precipitate is washed in due time with weak ammonia water. In general, however, as these conditions can seldom be fulfilled, they recommend to decant the supernatant liquid through the filter which is later to receive the precipitate, to dissolve this in as little hydrochloric acid as possible, to reprecipitate by dilute ammonia without further addition of magnesia mixture, and to wash finally with weakly ammoniacal water. Excess of ammonia, of ammonium salts, and of precipitant are all objectionable. In rock analysis the second precipitation will seldom be necessary. For ignition, etc., of the precipitate, see this subject under Magnesium (p. 66.)

\footnotetext{
${ }^{1}$ Am. Jour. Sci., 4th series, Vol. V1I, p. 187, 1899; Zeitschr. für anorg. Chemie, Vol, XX, p. 121,
} 1899; Chemical News, Vol. LXXIX, pp. 233, 244, 255, 1899. 
The following procedure admits of determining in the same portion, besides phosphorus, barium, iron, vanadium, chromium, and titanium, the last two either colorimetrically or gravimetrically, and is in large part extracted from a paper by Dr. T. M. Chatard. ${ }^{1}$

Silica is removed by hydrofluoric and sulphuric acids, excess of fluorine expelled, the residue brought into solution as far as possible with sulphuric or hydrochloric acid and hot water, filtered, the residue ignited, fused with sodium carbonate, dissolved in hydrochloric acid, and the solution, after precipitation of barium, added to the main one, which is now precipitated by ammonia to get rid of the magnesium salts usually present and thus insure a cleaner subsequent fusion with sodium carbonate.

The precipated $\mathrm{Al}_{2} \mathrm{O}_{3}, \mathrm{P}_{2} \mathrm{O}_{5}, \mathrm{Cr}_{2} \mathrm{O}_{3}, \mathrm{Fe}_{2} \mathrm{O}_{3}$, and $\mathrm{TiO}_{2}$ is dissolved in hot hydrochloric acid and filtered into a large platinum crucible, the filter burned and added, the solution evaporated to pastiness, a little water added to dissolve the salts, and dry sodium carbonate added in portions and stirred in thoroughly to prevent lumpiness in the fusion to follow, which is continued for half an hour. Addition of sodium nitrate is not necessary.

The fused mass is boiled out with water and washed with very dilute sodium-carbonate solution. In the residue iron and titanium can be determined by the methods already described. In the filtrate chromium can be determined colorimetrically if present in sufficient amount to give a pronounced color (see p. 80). Afterwards, or immediately if the chromium is not to be thus estimated, enough ammonium nitrate is added to react with all the carbonate and the solution is digested on the bath till most of the ammonium carbonate is gone. Nearly, if not quite all alumina is thus thrown out, carrying with it all phosphorus. The precipitate is washed with dilute ammonium-nitrate solution till the yellow color wholly disappears, after which it is dissolved in nitric acid and the phosphorus thrown out by molybdate solution. The filtrate, containing chromium and vanadium, can be treated as detailed in the next following sections.

\section{CHROMIUM.}

If vanadium is absent, or nearly so, as is apt to be the case in those highly magnesian rocks (peridotites) usually carrying a good deal of chromium, the following separation and gravimetric method for chromium gives good and concordant results, but in presence of vanadium, and it is best generally to assume its presence, the colorimetric method should always be adopted.

${ }^{1}$ Am. Chem. Jour. Vol. XIII, p. 106, 1891; Bull. U. S. Geol. Survey No. 78, p. 87; Chemical News, Vol. LXIII, p. 267, 1891. 


\section{GRAVIMETRIC METHOD.}

Having obtained chromium in solution as chromate and free from all else but a little alumina, as at the conclusion of the preceding section on phosphorus, proceed as follows:

Concentrate if necessary and add fresh ammonium sulphide, or introduce hydrogen sulphide. The chromium is reduced and appears as a precipitate of sesquioxide mixed with the rest of the alumina. This precipitate is now treated according to Baubigny ${ }^{1}$ by dissolving in nitric acid, evaporating to near dryness and heating with strong nitric acid and potassium chlorate, finally evaporating to dryness to get rid of the acid. Oxidation is complete and very speedy. On dilution with cold water, acid sodium carbonate is added in slight excess, and after two or three hours the precipitated alumina is filtered off. From the filtrate the chromium is then thrown out by fresh ammonium sulphide, redissolved and reprecipitated to free from alkali, and weighed.

The separation of aluminum from chromium by hydrogen peroxide in ammoniacal solution, as recommended by Jannasch and Cloedt, ${ }^{2}$ has been shown by Friedheim and Brühl, ${ }^{3}$ together with most of the other separations of Jannasch based on the use of hydrogen peroxide and from which so much was hoped, to be valueless.

\section{COLORIMETRIC METHOD.}

For this very accurate and by far the quickest method ${ }^{4}$ for determining chromium in rocks and ores where the amount does not exceed a few per cent, there is needed the aqueous extract of a sodium-carbonate fusion of the rock (as obtained, for instance, under Phosphorus, p. 79) in order to compare its color with that of a standard solution.

Preparation and strength of standard solution.-This standard solution is made by dissolving 0.25525 gram or double that amount of purre potassium monochromate in one liter of water made alkaline by a little sodium carbonate. Each cubic centimeter then corresponds to one-tenth milligram or two-tenths milligram of chromic oxide $\left(\mathrm{Cr}_{2} \mathrm{O}_{3}\right)$, in which condition chromium is usually reported in rocks and ores. It is probably inadmissible to increase the strength of the standard much above the figure given.

Preparation of the test solution.-Before filtering the aqueous extract of the sodium-carbonate fusion a few drops of alcohol (ethyl or methyl) are added to destroy the color of sodium manganate. If the yellow color of the filtrate is very faint, concentration by evaporation

${ }^{1}$ Bull. Soc. Chimique (n. s.) Vol. XLII, p. 291, 1884; Chemical News, Vol. L, p. 18, 1885.

2 Zeitschr. für anorg. Chemie, Vol. X, p. 402, 1895.

${ }^{3}$ Zeitschr. für anal. Chemie, Vol. XXXVIII, p. 681, 1899.

4 W. F. Hillebrand, Jour. Am. Chem. Soc., Vol. XX, p. 454, 1898; Chemical News, Vol. LXXVIII, pp. 227, 239, 1898; Bull. U. S. Geol. Survey No. 167, p. 37; First applied by L. de Koningh (Nederl. Tyds. voor Pharm. Chem. and Tox., 1889) for the estimation of chromium in foodstuffs. 
will strengthen it, and less than 2 milligrams of chromic oxide in 1 gram of rock can then be exactly measured. For smaller amounts it is best to employ from 3 to 5 grams of powder and then to concentrate the chromium by precipitation by mercurous nitrate, as detailed in the next section under Vanadium (p. 83); otherwise it may be difficult or impossible, because of the large amount of alkali carbonate present, to obtain a filtrate of sufficiently small bulk to show a decided color.

If niter has been used in the fusion and the crucible has been at all attacked by it, a yellow coloration of the filtrate may be due to dis. solved platinum, but neither the proportion of niter nor the temperature of the blast should ever be high enough to permit the crucible to be attacked.

Comparison of colors. - The final solution is transferred to a graduated flask of such size that its color shall be weaker than that of the standard chromium solution. Definite amounts of the latter are then diluted with water from a burette until of the same strength as the test solution, exactly as described on page 68 for the colorimetric estimation of titanium. For very minute amounts it is necessary to use Nessler tubes, as in ammonia estimations, instead of the glasses and apparatus described and depicted under Titanium (p. 70).

As with colorimetric methods in general, this one gives better results with small than with large percentages of chromium, yet it can be applied in the latter cases with satisfactory results by making a larger number of consecutive comparisons with the same solution.

\section{a FEW COMPaRative Data.}

A few comparisons between colorimetric and gravimetric determinations of chromium are here given to show the order of agreement, the former having been made several months and even years after the latter.

\begin{tabular}{|r|c|}
\hline $\begin{array}{c}\text { Gravimetric } \\
\text { per cent } \mathrm{Cr}_{2} \mathrm{O}_{3} .\end{array}$ & $\begin{array}{c}\text { Colorimetric } \\
\text { per cent } \mathrm{Cr}_{2} \mathrm{O}_{3} .\end{array}$ \\
\hline Trace. & 0.018 \\
0.05 & .051 \\
.14 & .12 \\
.08 & .033 \\
Trace. & .013 \\
None. & .0086 \\
None. & .0067 \\
\hline
\end{tabular}

The outcome was somewhat surprising, for it was hardly to be expected that the long and laborious quantitative separations should Bull. $176-6$ 
have resulted so well as they are shown to have done. It should be mentioned that for the gravimetric tests but one or two grams at most were used, which accounts for the reported absence of chromium in two instances, this report being based on the lack of color in the aqueous extract of the alkali fusion after removal of manganese.

\section{VANADIUM (CHROMIUM) AND MOLYBDENUM.}

\section{DISTRIBUTION OF VANADIUM AND MOLYBDENU'̃.}

The wide distribution of vanadium thoughout the earth's crust has in recent years been clearly established (see ante, p. 18), not only in ores and in coals, but in clays, limestones, sandstones, and igneous rocks. ${ }^{1}$ The writer has shown (loc. cit.) that vanadium occurs in quite appreciable amounts in the more basic igneous and metamorphic rocks up to 0.08 per cent or more of $\mathrm{V}_{2} \mathrm{O}_{3}$, but that it seems to be absent, or nearly so, from the highly siliceous ones. Some of their ferric aluminous silicate constituents carry still higher percentages-up to 0.13 per cent $\mathrm{V}_{2} \mathrm{O}_{3}$ in a biotite separated from a pyroxenic gneiss. Molybdenum, on the other hand, appears to be confined in quantities susceptible of detection to the more siliceous rocks, and, except perhaps in rare instances, is not present in them in quantitatively determinable amount when operating on 5 grams of material. Hence the quantitative search for vanadium will usually be limited to rocks with less than 60 per cent of silica. The search for it even then will perhaps not often warrant the necessary expenditure of time, but in this connection it is to be remembered that neglect to estimate it introduces an error in the figures for both ferrous and ferric oxides, which in extreme cases met with may be of considerable moment. (See p. 58, and also pp. 95-96.)

\section{DESCRIPTION OF METHOD.}

In the following method there is nothing absolutely novel except that chromium and vanadium, when together, need not be separated, but are determined, the former colorimetrically, as already described (p. 80), the latter volumetrically, in the same solution. ${ }^{2}$

Five grams of the rock are thoroughly fused over the blast with 20 of sodium carbonate and 3 of sodium nitrate. After extracting with water and reducing manganese with alcohol it is probably quite unnecessary, if the fusion has been thorough, to remelt the residue

1 W. F. Hillebrand, Distribution and quantitative occurrence of vanadium and molybdenum in rocks of the United States: Am. Jour. Sci., 4th series, Vol. VI, p. 209, 1898; Chemical News, Vol. LXXVIII, p. 216, 1898; Bull. U. S. Geol, Survey No. 167, p. 49.

2 W. F. Hillebrand: Jour. Am. Chem. Soc., Vol. XX, p. 461, 1898; Chemical News, Vol. LXXVIII, p. 295, 1898; Bull. U. S. Geol. Surv., No. 167, p. 44. 
as above, though for some magnetites and other ores containing larger amounts of vanadium than the generality of rocks, this may be necessary, as Edo Claassen has shown. ${ }^{1}$ The aqueous extract is next nearly neutralized by nitric acid, the amount to be used having been conveniently ascertained by a blank test with exactly 20 grams of sodium carbonate, etc., and the solution is evaporated to approximate dryness. Care should be taken to avoid overrunning neutrality, because of the reducing action of the nitrous acid set free from the nitrite produced during fusion, but when chromium is present it has been my experience that some of this will invariably be retained by the precipitated silica and alumina, though only in one case have I observed a retention of vanadium, it being then large. The use of ammonium nitrate instead of nitric acid for converting the sodium carbonate into nitrate does not seem to lessen the amount of chromium retained by the silica and alumina.

As a precautionary measure, therefore, and always when chromium is to be estimated also, the silica and alumina precipitate should be evaporated with hydrofluoric and sulphuric acids, the residue fused with a little sodium carbonate and the aqueous extract again nearly neutralized with nitric acid and boiled for a few moments, the filtrate being added to the main one.

Mercurous nitrate is now added to the cold alkaline solution in some quantity, so as to obtain a precipitate of considerable bulk, containing, besides mercurous carbonate, chromium, vanadium, molybdenum, tungsten, phosphorus, and arsenic, should all happen to be in the rock. The mercurous carbonate serves to counteract any acidity resulting from the decomposition of the mercurous nitrate. Precipitating in a slightly. alkaline instead of a neutral solution, renders the addition of precipitated mercuric oxide unnecessary for correcting this acidity. If the alkalinity, as shown by the formation of an unduly large precipitate, should have been too great, it may be reduced by careful addition of nitric acid until an added drop of mercurous nitrate no longer produces a cloud.

After heating and filtering, the precipitate is ignited in a platinum crucible, after drying and removing from the paper to obviate any chance of loss of molybdenum and of injury to the crucible by reduction of arsenic. The residue is fused with a very little sodium carbonate, leached with water, and the solution, if colored yellow, filtered into a graduated flask of 25 or more cubic centimeters capacity. The chromium is then estimated accurately in a few minutes by comparing with a standard alkaline solution of potassium monochromate (p. 80). Then, or earlier in absence of chromium, sulphuric acid is added in slight excess and molybdenum and arsenic, together with occasional traces of platinum, are precipitated by hydrogen sulphide, preferably

${ }^{1}$ Am: Chem. Jour., Vol. VIII, p. 437, 1886. 
in a small pressure bottle. ${ }^{1}$ If the color of the precipitate indicates absence of arsenic the filter with its contents is carefully ignited in porcelain, and the delicate sulphuric-acid test for molybdenum is applied as follows: The molybdenum compound is heated in porcelain with a single drop of strong sulphuric acid till the acid is nearly vola: tilized. On cooling a beautiful blue color is proof of the presence of molybdenum.

The filtrate, in bulk from $25 \mathrm{~cm} .{ }^{3}$ to $100 \mathrm{~cm} .{ }^{3}$ is boiled to expel hydrogen sulphide, and titrated at a temperature of $70^{\circ}$ to $80^{\circ} \mathrm{C}$. with a very dilute solution of permanganate representing about $1 \mathrm{mg}$. of $\mathrm{V}_{2} \mathrm{O}_{5}$ per cubic centimeter as calculated from the iron strength of the permanganate, one molecule of $\mathrm{V}_{2} \mathrm{O}_{5}$ being indicated for each one of $\mathrm{Fe}_{2} \mathrm{O}_{3}$. One or two checks are always to be made by reducing again by means of a current of sulphur dioxide gas, boiling this out again, ${ }^{2}$ and repeating the titration. The latter results are apt to be a very little lower than the first and are to be taken as the correct ones.

In case the volume of permanganate used is so small as to make doubtful the presence of vanadium, it is necessary to apply a qualitative test, which is best made as follows: The solution is evaporated and heated to expel excess of sulphuric acid, the residue is taken up with two or three cubic centimeters of water and a few drops of dilute nitric acid, and a couple of drops of hydrogen peroxide are added. A characteristic brownish tint indicates vanadium. Unless the greater part of the free sulphuric acid has been removed the appearance of this color is sometimes not immediate and pronounced, hence the above precaution. It is also necessary that the nitric acid shall be in considerable excess, since in a neutral or only faintly acid solution the color does not appear strongly.

The above is a surer test to apply than the following: Reduce the bulk to about 10 cubic centimeters, add ammonia in excess and introduce hydrogen sulphide to saturation. The beautiful cherry-red color of vanadium in ammonium-sulphide solution is much more intense than that caused by hydrogen peroxide in acid solution, but the action of ammonia is to precipitate part or all of the vanadium with the chromium or aluminum that may be present or with the manganese used in titrating, and ammonium sulphide is unable to extract the vanadium wholly from these combinations. Usually, however, the

\footnotetext{
1 From a sulphuric solution the separation of platinum and molybdenum by hydrogen sulphide is much more rapid and satisfactory than from a hydrochloric solution.

2The direct use of a solution of sulphur dioxide or of an alkali sulphite is inadmissible unless these have been freshly prepared, since after a lapse of time they contain other oxidizable bodies than sulphurous acid or a sulphite. The sulphur dioxide is best obtained as wanted by heating a flask containing a solution of sulphur dioxide, or of a sulphite to which sulphuric acid has been added.

The expulsion of the last traces of sulphur dioxide is said to be more effectively accomplished by boiling with simultaneous passage of a rapid current of carbon dioxide for a few minutes at the last than by boiling alone. Because of the small amount of air carried with it, long passage of the gas is said to result in slight oxidation of the vanadium (Manasse, Ann. Chem, u. Pharm., Vol. CCXL, p. 23, 1887; Zeitschr. für anal. Chemie, Vol. XXXII, p. 225, 1893).
} 
solution will show some coloration, and addition of an acid precipitates brown vanadium sulphide, which can be collected, ignited, and further tested if desired.

application of the method in PResence of Relatively much Chromum.

The application of the method in its foregoing simplest form is subject to one limitation-the chromium must not be present above a certain moderate amount. This limitation is due to the considerable amount of permanganate then required to produce a clear transition tint when titrating in a hot solution, as is advisable with vanadium. In a cold solution of chromic sulphate much less permanganate is needed to produce the peculiar blackish tint without a shade of green, which affords a sure indication of excess of permanganate, but in a hot and especially a boiling solution the oxidation of the chromium itself takes place so rapidly that a very large excess of the reagent may be added before a pronounced end reaction is obtained. Nevertheless, quite satisfactory determinations of as little as one or two milligrams of vanadium pentoxide can be made in presence of as much as 30 milligrams of chromic oxide. To accomplish this it is only necessary to apply a simple correction obtained by adding permanganate to a like bulk of equally hot chromic sulphate solution containing approximately the same amount of chromium.

Ridsdale ${ }^{1}$ titrated the cold solution to avoid oxidation of chromium, and obtained accurate results, but in the writer's experience the end reaction is then uncertain. 
. 86 SOME PRINCIPLES AND METHODS OF ROCK ANALYSIS. [BULL, 176.

The following tables contain the results of a considerable number of tests, those in Table II being tabulated separately in order to show the degree of accuracy attainable with a large excess of chromium by applying the correction above mentioned and also the amount of this correction:

Table I.

Tests for vanadium in the presence of chromium.

\begin{tabular}{|c|c|c|c|c|}
\hline No. & $\begin{array}{l}\text { Chromic } \\
\text { oxide. }\end{array}$ & $\begin{array}{l}\text { Vanadium } \\
\text { pentoxide. }\end{array}$ & $\begin{array}{l}\text { Vanadium } \\
\text { pentoxide } \\
\text { found. }\end{array}$ & Error. \\
\hline & Milligrams. & Milligrams. & Milligrams. & Mrilligram. \\
\hline 1 & 1 & 9.87 & 9.22 & -0.15 \\
\hline \multirow{2}{*}{2} & 1 & .94 & 1.04 & +.10 \\
\hline & & & .98 & +.04 \\
\hline \multirow[t]{2}{*}{3} & 1.5 & 5.25 & 5.49 & +.24 \\
\hline & & & 5.43 & +.19 \\
\hline \multirow[t]{2}{*}{4} & 2 & 5.62 & 5.5 & -.12 \\
\hline & & & 5.5 & -.12 \\
\hline \multirow[t]{3}{*}{5} & 3 & 4.68 & 4. 78 & +.10 \\
\hline & & & 4. 78 & +.10 \\
\hline & & & 4.83 & +.15 \\
\hline \multirow[t]{2}{*}{6} & 3 & 5.62 & 5.58 & -.04 \\
\hline & & & 5. 58 & -.04 \\
\hline \multirow[t]{2}{*}{7} & 3.5 & 18.74 & 18. 89 & +.15 \\
\hline & & & 18.97 & +.23 \\
\hline 8 & 6 & 5.6 & 6.1 & +.50 \\
\hline 9 & 6 & 4. 68 & 4.78 & +.10 \\
\hline 10 & 6 & 5.62 & 5.58 & -.04 \\
\hline 11 & 10 & 5. 62 & 5.58 & -.04 \\
\hline \multirow[t]{2}{*}{12} & 10 & 23.52 & 23.81 & +.29 \\
\hline & & & 23.71 & +.19 \\
\hline \multirow[t]{2}{*}{13} & 10 & 46.85 & 46.98 & +.13 \\
\hline & & & 47.20 & +.35 \\
\hline \multirow[t]{2}{*}{14} & 25 & 23.52 & 23.65 & +.13 \\
\hline & & & 23.75 & +.23 \\
\hline 15 & 87.5 & 23.52 & 23.71 & +.19 \\
\hline
\end{tabular}


TABLe II.

Showing application of correction for larger amounts of chromium, obtained by adding potassium permanganate to an equal bulk of solution containing a like amount of chromic sulphate.

\begin{tabular}{|c|c|c|c|c|c|c|}
\hline No. & $\begin{array}{l}\text { Chromic } \\
\text { oxide. }\end{array}$ & $\begin{array}{l}\text { Vanadium } \\
\text { pentoxide. }\end{array}$ & $\begin{array}{l}\text { Vanadium } \\
\text { pentoxide } \\
\text { found. }\end{array}$ & $\begin{array}{l}\text { Vanadium } \\
\text { pentoxide } \\
\text { found. }\end{array}$ & Error. & $\begin{array}{l}\text { Volume of solu- } \\
\text { tion. }\end{array}$ \\
\hline 16 & $\begin{array}{c}\text { Milligrams. } \\
20\end{array}$ & $\begin{array}{c}\text { Milligrams. } \\
0.94\end{array}$ & $\begin{array}{c}\text { Uncorrected. } \\
1.59\end{array}$ & $\begin{array}{c}\text { Corrected. } \\
0.99\end{array}$ & $\begin{array}{c}\text { Ifilligram. } \\
+0.05\end{array}$ & $50-100 \mathrm{~cm}^{3}$ \\
\hline \multirow[t]{3}{*}{17} & \multirow[t]{3}{*}{20} & \multirow[t]{3}{*}{1.87} & 2.69 & 2.09 & +.22 & $50-100 \mathrm{~cm}^{.3}$ \\
\hline & & & 2.39 & 1.79 & -.08 & . \\
\hline & & & 2.59 & 1.99 & +.12 & \\
\hline \multirow[t]{3}{*}{18} & \multirow[t]{3}{*}{20} & \multirow[t]{3}{*}{18.74} & 19.4 & 18.73 & -.01 & $50-100 \mathrm{~cm}^{3}$ \\
\hline & & & 19.3 & 18. 63 & -.11 & \\
\hline & & & 19.3 & 18.63 & -.11 & \\
\hline \multirow[t]{5}{*}{19} & \multirow[t]{5}{*}{30} & \multirow[t]{5}{*}{1.87} & 2.99 & 2.14 & +.27 & About $100 \mathrm{~cm}^{3}$ \\
\hline & & & 2.79 & 1.94 & +.07 & \\
\hline & & & 2.79 & 1.94 & +.07 & \\
\hline & & & 2.69 & 1.84 & -.03 & \\
\hline & & & 2.69 & 1.84 & -.03 & \\
\hline \multirow[t]{4}{*}{20} & \multirow[t]{4}{*}{30} & \multirow[t]{4}{*}{1.87} & 2.69 & 1.79 & -.08 & $200 \mathrm{~cm}^{3}$ \\
\hline & & & 2.89 & 2.09 . & +.22 & \\
\hline & & & 2.89 & 2.09 & +.22 & \\
\hline & & & 2.79 & 1.99 & +.12 & \\
\hline 21. & 62 & 46.85 & 48. 60 & 47.60 & +.75 & $200 \mathrm{~cm}^{3}{ }^{3}$ \\
\hline
\end{tabular}

In spite of the fact that the correcion in most of the trials of this last table represents a large proportion of the permanganate used, the results must be considered satisfactory in view of the small amount of vanadium present, and they show that the method in competent hands after a little experience affords trustworthy figures.

The method of T. Fischer ${ }^{1}$-digestion of the precipitated lead salts with a strong solution of potassium carbonate-appears to offer the long-needed satisfactory quantitative separation of arsenic, phosphorus, chromium, tungsten, and molybdenum from vanadium, the normal lead meta-vanadate remaining quite unattacked, according to the author, while the other lead salts are wholly decomposed, but the applicability of this method to the separation of the minute amounts often found in rocks and ores has not been tested. The object has been in the present case to reach satisfactory results with the greatest expedition, and when chromium is not present in considerable amount this is accomplished.

Fortunately, chromium is almost never a prominent constituent of 
clays, coals, iron ores, and those rocks in which vanadium has thus far been reported, for although it is usually certain of the most basic of the silicate rocks that are highest in chromium - as the peridotitesyet in these, so far as present experience teaches, vanadium is lacking, a fact doubtless connected with the simultaneous absence from them of ferric-aluminous silicates.

\section{CONDITION OF VANADIUM IN ROCKS.}

The above and elsewhere mentioned connection of vanadium with the ferric-aluminous silicates of rocks, taken in connection with the existence of the mineral roscoelite, classed as a vanadium mica, indicates a condition of the vanadium corresponding to aluminum and ferric iron, and that it is to be regarded as replacing one or both of these elements. Hence it should be reported as $\mathrm{V}_{2} \mathrm{O}_{3}$ and not as $\mathrm{V}_{2} \mathrm{O}_{5}$.

What its condition may be in matter of secondary origin, like clays, limestones, sandstones, coals, and ores of iron, is yet open to discussion. It was the writer's opinion until recently, that it should then be regarded as in the pentavalent state $\left(\mathrm{V}_{2} \mathrm{O}_{5}\right)$, but his work upon certain remarkable vanadiferous sandstones ${ }^{1}$ of western Colorado, in which it unquestionably occurs as trivalent vanadium $\left(\mathrm{V}_{2} \mathrm{O}_{3}\right)$, has led to a decided unsettling of this view. It is but proper to recall that Czudnowicz; ${ }^{2}$ because of the extreme difficulty in completely extracting it from iron ores by an alkali-carbonate fusion and because of the easy reducibility of vanadic acid by ferrous salts, under the conditions in which brown iron ores are supposed to form, considered the vanadium in such ores to be in a lower condition of oxidation $\left(\mathrm{V}_{2} \mathrm{O}_{3}\right)$. Lindemann's ${ }^{3}$ contrary conclusion with regard to certain iron ores, because the vanadium was extracted as $\mathrm{V}_{2} \mathrm{O}_{5}$ by sodium-carbonate fusion without niter, is not valid, since this would probably be the case even if it existed in the ore as $\mathrm{V}_{2} \mathrm{O}_{3}$.

XIX. FERROUS IRON.

COMPARISON OF SEALED-TUBE AND HYDROFLUORIC-ACID METHODSCOMPARATIVE WORTHLESSNESS OF THE FORMER IN ROCK ANALYSIS.

No point in rock analysis has been the cause of greater solicitude to the chemist, and especially to the mineralogist and petrographer, than the determination of iron in ferrous condition. The sealed-tube or Mitscherlich method .with sulphuric acid, for a long time the only available onè, is in theory perfect, since complete exclusion of oxygen is easily attainable. Its chief hitherto recognized defect lies in the inability to always secure complete decomposition of the iron-bearing minerals, and even to ascertain, oftentimes, whether or not the decom-

1 Hillebrand and Ransome: Am. Jour. Sci., 4th series, Vol. X, p. 120, 1900.

2 Pogg. Ann., Vol. CXX, p. 20, 1863.

${ }^{3}$ Dissertation, Jena. 1878, through Zeitsch. für anal. Chemie, Vol. XVIII, p. 99, 1879. 
position has been complete. The addition of hydrofluoric acid to the sulphuric in the tube, in order to insure this breaking up, is to be regarded as of very doubtful utility in most cases, since the glass may be so strongly attacked as to add an appreciable amount of iron to the solution, and the hydrofluoric acid may have exhausted itself in attacking the glass before the more refractory minerals succumb. Nevertheless, if decomposition can be effected by sulphuric acid alone the results obtained are sharp and concordant, and what has seemed especially remarkable, and up to almost the present moment without a satisfactory explanation, they are in rock analysis usually higher than when made by any of the modifications of the hydrofluoric-acid method now. so extensively practiced. This difference is not very marked with rocks containing but 1 or 2 per cent of ferrous iron, but it increases with rising percentage to such an extent that where the sealed-tube method will show 12 per cent $\mathrm{FeO}$ the other may indicate no more than 10 per cent. This is a fact of which the writer has long been cognizant, but it does not seem to be known to chemists or petrographers at large, though Wülfing ${ }^{1}$ has noticed this difference in certain analyses without appreciating its significance. Experiments with soluble iron salts of known composition, like ferrous sulphate and ferrous-ammonium sulphate, throw no light on the subject, for both methods give with them the same sharp and accurate results.

In spite of several attempts to find a solution to the problem, none presented itself until very recently, when, as a result of observations made in this laboratory by $\mathrm{Dr}$. H. N. Stokes in a pending investigation on the action of ferric salts on pyrite and other sulphides, the clue was given. Dr. Stokes found that ferric salts exercise a most marked oxidizing effect on pyrite and probably other sulphides. The reaction is not new (see J. H. L. Vogt in Zeitschr. für prakt. Geol., 1899, pp. 250-251), but the ease with which the change takes place and the completeness of the oxidation of the pyrite, not only of its iron but of the greater part of the sulphur as well, were entirely unexpected. Pure pyrite itself is attacked with extreme slowness by boiling dilute sulphuric and hydrofluoric acids, either alone or mixed, but the moment a ferric salt is introduced the case. is altogether different.

However, experiment has shown (p. 95) that with the amounts of sulphides usually found in igneous rocks their effect upon the estimation of ferrous iron by the hydrofluoric-acid method at atmospheric pressure and boiling heat is negligible, though by increasing the amount of sulphide the effect becomes more and more apparent, because of the greater surface of pyrite exposed to the action of the ferric iron of the rock.

Under the conditions of the Mitscherlich method, on the other handa temperature of 150 to $200^{\circ} \mathrm{C}$, and even higher, high pressure, much longer time of action, and impossibility of escape of any hydrogen sul-

1 Ber. deutsch. chem. Gesell., Vol. XXXII, p. 2217, footnote, 1899. 
phide that may be formed-the sulphur of the sulphides becomes nearly, if not fully oxidized to sulphuric acid at the expense of the ferric iron in the rock, with the production of an equivalent amount of ferrous iron in addition to that resulting from the sulphide itself. Now, rocks with hardly an exception, and many minerals, carry pyrite or pyrrhotite, or both, often in considerable amount, often in traces only. My own experience has been that sulphur can almost always be detected in 2 grams of rock powder.

Let us now see what the effect of these traces when fully oxidized amounts to. One atom of sulphur (32) requires for its complete conversion to trioxide the oxygen of three molecules of ferric oxide (480), which then becomes six molecules of ferrous oxide (432). In other words, 0.01 per cent of sulphur may cause the ferrous oxide to appear too high by 0.135 per cent, and 0.10 per cent of sulphur may bring about an error of 1.35 per cent in ferrous oxide. The case is still worse if the sulphur is set free as hydrogen sulphide from a soluble sulphide, for then the above percentages of sulphur produce errors of 0.18 and 1.8 per cent, respectively, in the ferrous oxide determination.

The error caused by sulphides tends to become greater the more there is present of either or both sulphide and ferric salt. Now, the highly ferruginous rocks usually carry more ferric iron than the less ferruginous ones, and they are often relatively high in pyrite and pyrrhotite; hence the increasing discrepancy between the results by the two methods as the iron contents of the rocks rise is fully in accord with the above explanation. ${ }^{1}$

Notwithstanding that the Mitscherlich method'has thus been discredited in its general applicability to rocks and minerals, it is still capable of affording valuable assistance with those which are totally free from sulphides. Hence the conditions under which success can best be achieved by it are set forth in the following paragraphs.

THE MODIFIED MITSCHERLICH . METHOD.

Strength of acid.-The method in its original and usual application calls for a mixture of three parts of sulphuric acid and one of water by weight, or about three to two by volume, though a still stronger acid is sometimes used. In some cases, however, perhaps in most, much better decomposition of the silicates is effected by reversing the proportions of water and acid, or at any rate by diluting considerably beyond the above proportion. Hereby the separation of salts difficultly soluble in the stronger acid is avoided and the actual solvent effect on the minerals seems to be in no wise diminished.

\footnotetext{
1 For details of experiments showing the worthlessness of the Mitscherlich method for rocks and minerals which contain even a trace of free sulphur or sulphides, see Hillebrand and Stokes, in an as yet unpublished paper in Jour. Am. Chem. Soc., Vol, XXII, and Zeitschr. füranory. Chem., Vol. XXV, 1900, entitled, Relative value of the Mitscherlich and hydrofluoric-acid methods for ferrous iron determinations.
} 
Filling, sealing, and heating of the tube.-The very finely powdered mineral having been introduced into a tube of resistant glass free from ferrous iron, the open end is drawn out, so as to leave a funnel for the introduction of the acid. A very little water is then introduced and carefully heated to boiling for a moment to expel all air from the powder. The diluted acid-which has just been boiled down from a state of greater dilution in order to have it free from air-is then poured in until the tube is about three-fourths filled. Carbon dioxide is then introduced from a generator which has been in active operation for some time, through a narrow glass tube drawn out of the same kind of glass as that of which the decomposing tube consists. In a few moments the air is expelled, and the small tube is then sealed into the large one over the blast lamp without interrupting the gas current until the very last instant, when to prolong it would perhaps cause a blowing out of the softened glass. The interruption of the current at the proper moment is easily effected by the pressure of the thumb and finger holding the small tube at the point where it enters the rubber tube leading from the gas generator. No breakage in the oven ever occurs as a consequence of thus fusing one tube into the other.

The heating is done in a bomb oven at any desired temperature up to, say, $200^{\circ} \mathrm{C}$, and continued at intervals until examination by aid of a low-power lens shows that decomposition is complete or has progressed as far as can be hoped for. By inclosing the glass in an outer tube of strong steel, properly capped ${ }^{1}$ and containing a little ether or benzine to equalize the pressure on both sides of the glass, the temperature can be elevated far beyond what is otherwise permissible, and the decomposition will then doubtless be more complete with refractory silicates.

Reason for introducing gas and sealing as above directed.-The usual practice in employing the above method has been to expel air before-sealing by introducing a few crystals or lumps of an alkali carbonate or bicarbonate, the gas set free on their contact with the acid being supposed to effectively expel all air. That this is not accomplished the following series of comparative results long since published elsewhere ${ }^{2}$ fully show. The material used was the oxide of uranium $\mathrm{U}_{3} \mathrm{O}_{8}$, requiring by theory 32.07 per cent of $\mathrm{UO}_{2}$. Operating as just above described on from 0.3 to 0.5 gram, the results were

$$
31.06,31.07,29.72,29.33,29.89,30.69 \text {, }
$$

whereas after filling the tube with gas from a generator there was found

$$
32.11,31.90,32.15,32.12,32.06,32.17,32.28
$$

the average error of the former series being 1.78 per cent. The percentage error would, of course, be reduced by increasing the weight

\footnotetext{
1 Ullmann, Zeitschr. für angew. Chemie, 1893, p. 274; Zeit. für anal. Chemie, Vol. XXXIII, p. 582, 1894.

2 Bull. U. S. Geol. Survey, No. 78, p. 50; Chemical News, Vol. LXIV, p. 232, 1891.
} 
of mineral operated on. An average error equal to the above when employing 1 gram of ferrous minerals would make the percentage for $\mathrm{FeO}$ about 0.3 per cent too low. While the absolute error might be the same in all cases, the relative error would increase with minerals low in ferrous iron.

THE HYDROFLUORIC-ACID METHOD.

This method is the one which has been almost exclusively used since the earliest years of the Survey's existence.

The specially ground powder, in a capacious crucible, is placed, after stirring up with dilute sulphuric acid, on a small water bath of a single opening (fig. 13) and covered with a glass funnel, the stem of which has been cut off near the flare, resting in a depression of the specially made cover, into which water constantly drops from a tubulated bottle, thus securing a perfect water joint and serving to keep the bath full. Through a small metal pipe carbonic-acid gas flows into the bath above the surface of the water, and rising through orifices in the cover fills the funnel and crucible. ${ }^{1}$ The lamp under the bath is lighted and hydrofluoric acid is poured into the crucible through a platinum funnel, which is left in place to serve as an occasional stirrer, for which a rod or wire may be substituted. After boiling commences the rapid gas current can be safely interrupted, to be restored when the lamp is extinguished after one-half to one or more hours. A full stream of cold water is then caused to flow from the tubulated bottle into the bath, the overflow from the outlet tube being caught in a receiver. As soon as cool the contents of the crucible are emptied into a platinum dish containing cold water, and titrated till the first permanent color appears, which usually will last for only a few seconds. Duplicate determinations are to be advised whenever possible, since even with the utmost care the results will occasionally differ more than is allowable.

In absence of a suitable water bath an ordinary one can be used covered with a beaker, through a hole in the bottom of which a strong current of carbon dioxide is introduced, or the crucible may be set in a sand bath and covered in the same way with a broken beaker (Doelter).

The cause of the rapid disappearance of the first pink blush when titrating in hydrofluoric-sulphuric solution appears to be the ready oxidizability of manganous fluoride by permanganate. The latter can be added by the cubic centimeter to solutions already containing manganous sulphate in presence of hydrofluoric acid without producing a more than passing pink blush. The solution, however, takes on in ever-increasing intensity the red-brown color characteristic of man. 
ganic salts. The decolorization due to this cause is hence much more pronounced in the case of rocks high in ferrous iron than of those low in this constituent, because of the greater amount of manganous salt

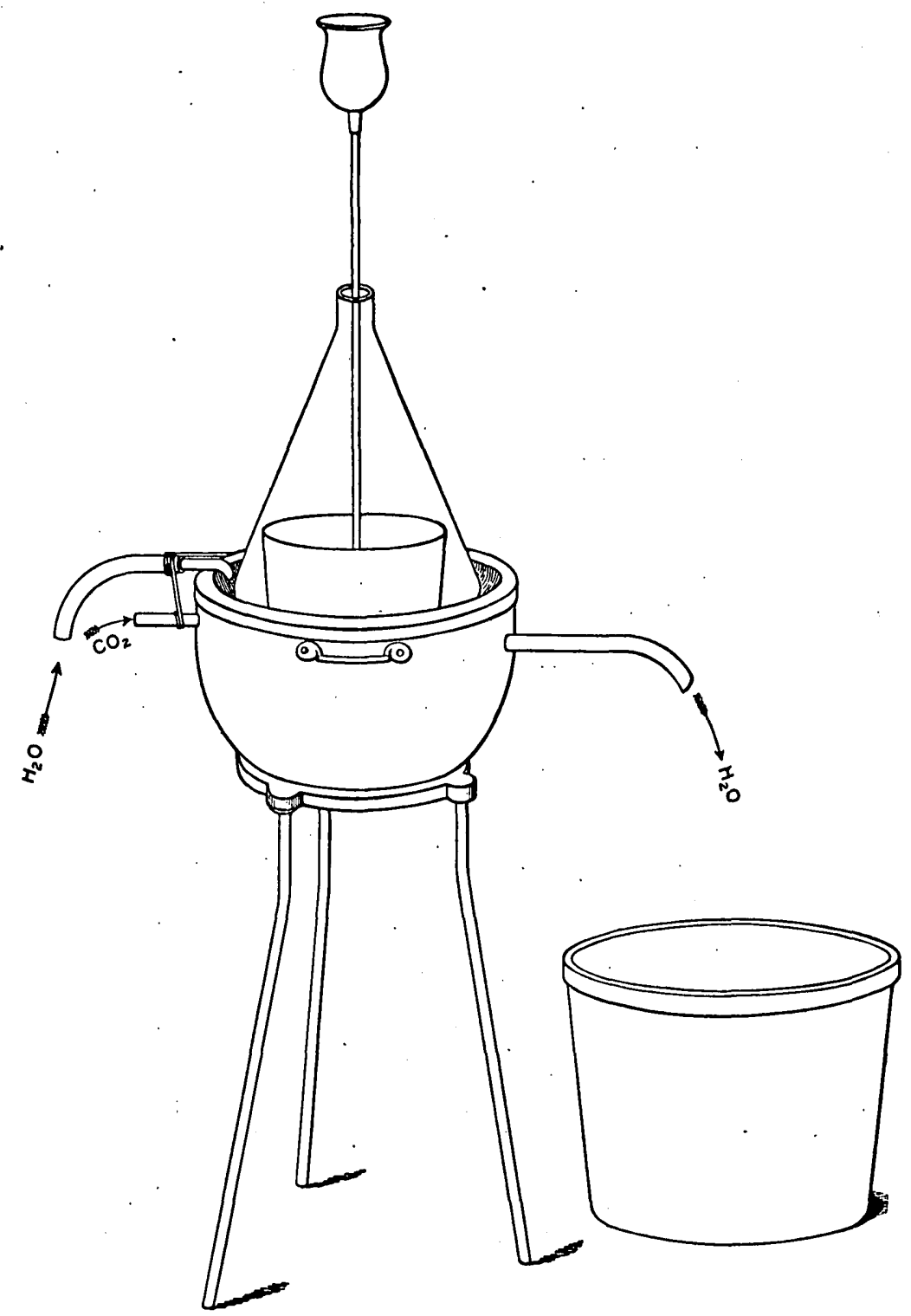

Fic. 13.-Cooke's apparatus for the determination of ferrous iron.

resulting from reduction of a correspondingly larger amount of permanganate.

When pyrite is present the bleaching is in part due to its action on any permanganate added in excess of the requirements of the ferrous 
oxide. But this action is not so immediate as to affect the ferrous oxide determination if the end point in the latter has been properly observed.

PRATT'S MODIFICATION OF THE HYDROFLUORIC-ACID METHOD.

J. H. Pratt ${ }^{1}$ has shown that very satisfactory ferrous iron determinations can be secured by simple boiling of the rock powder with hydrofluoric and sulphuric acids in a large crucible fitted with a cover and platinum tube for introduction of carbon dioxide. His test experiments on ferrous sulphate show that there need be practically no oxidation, even if the heating lasts several hours. The directions given on page 150 of his paper, with reference to the treatment of very refractory minerals which are not fully decomposed by this treatment, must be understood as referring only to homogeneous minerals and not to rocks, where the relations of ferrous and ferric iron in the undecomposed portion are certainly different from those in the part dissolved.

INFLUENCE OF SULPHIDES, VANADIUM, AND CARBONACEOUS MATTER ON

THE DETERMINATION OF FERROUS IRON BY THE HYDROFLUORIC-ACID METHOD.

A dark color of the undissolved resiaue may be due to pyrite, graphite, or carbonaceous matter. The first of these affects the result but little, the second probably not at all, and they can be distinguished by their behavior toward nitric acid. Organic matter of course renders impossible the estimation of ferrous iron.

Sulphides.-Pyrite, in the quantities usually met with in igneous rocks, is probably without serious effect on the ferrous-iron determination by any of the hydrofluoric-acid methods. This sulphide is very resistant toward attack in the absence of oxygen, as is shown by the fact that if present in any quantity it can be readily recognized in the residue after titration. In any case it is impossible to allow for an error introduced by its possible decomposition, and the result of titration must count as ferrous iron. In the case of soluble sulphides two sources of error are introduced-that of reduction of ferric iron by hydrogen sulphide evolved, and that due to the ferrous iron which the sulphides themselves may contain, especially if pyrrhotite is present. The first of these is perhaps negligible, since most of the hydrogen sulphide would probably be expelled without reducing iron. The second is approximately measurable if it is known that pyrrhotite is the only soluble sulphide present, and its amount has been ascertained by determining the hydrogen sulphide set free on boiling with hydrochloric acid in a current of carbon dioxide. In this case a correction is to be 
applied to the result of titration for total ferrous.iron. (See also p. 106, under Sulphur.)

In order to obtain quantitative data regarding the effect of pyrite on the ferrous-iron estimation by the hydrofluoric-acid method the following tests were recently made: Parc of a fine crystal of pyrite was rather finely powdered and boiled with dilute sulphuric acid, which extracted considerable ferrous iron, derived presumably from admixed or intergrown pyrrhotite, since a second boiling with fresh acid gave a negative test for ferrous iron. After washing by decantation with water, followed by alcohol and ether, the powder was dried and further pulverized. A quarter of a gram of it when treated with hydrofluoric and sulphuric acids in a large crucible by the Cooke method for ferrous iron, then rapidly filtered through a very large perforated platinum cone fitted with filter paper, required but 2 drops of a permanganate solution representing only 0.0032 gram $\mathrm{FeO}$ to the cubic centimeter.

Since, however, Dr. H. N. Stokes has found in a pending investigation that the oxidizing effect of ferric salts on pyrite and other sulphides is vastly greater than seems to have been suspected (see p. 89), the following tests were made in order to ascertain the probable error due to this action under the conditions prevailing in rock analysis: Successive portions of 1 gram each of a hornblende-schist, free from sulphur and carrying 10.09 per cent $\mathrm{FeO}$ as the méan of several determinations and 4.00 per cent $\mathrm{Fe}_{2} \mathrm{O}_{3}$, were mixed in a large $\left(50 \mathrm{~cm} .^{3}\right)$ platinum crucible with $0.02,0.025$, and 0.10 gran, respectively, of the above purified pyrite powder, and treated with hydrofluoric and sulphuric acids by the Cooke method, the water bath being at boiling heat for one hour. The cooled contents of the crucible were poured into a platinum dish containing water and titrated rapidly nearly to an end. Then, in order to get rid of the pyrite, which would obscure the end reaction by its reducing effect on the pemanganate, the solution was filtered as above and in the clear filtrate the titration was carried to completion. The results were 10.02, 10.16, and 10.70. Inasmuch as the smallest of these three charges of pyrite was several times greater than what may be considered an unusually high amount for an igneous rock, it is very evident that for all practical purposes the influence of pyrite on the ferrous estimation by the Cooke method is negligible. At the same time it is to be borne in mind that with increased content in ferric iron an increased amount of pyrite will be attacked, and that the extent of this attack is undoubtedly influenced by the degree of fineness of the pyrite powder.

Vonadium.-If vanadium, when present, exists in the trivalent condition, it necessarily affects with an error varying with its amount the result of titration for ferrous iron. Knowing the amount of vanadium a correction can be applied as follows: One molecule of $\mathrm{V}_{2} \mathrm{O}_{3}(150.8)$ 
in oxidizing to $\mathrm{V}_{2} \mathrm{O}_{5}$ requires as much oxygen as four molecules of $\mathrm{FeO}(288)$ when oxidized to $\mathrm{Fe}_{2} \mathrm{O}_{3}$. The proportion, 150.8: $288:: \mathrm{V}_{2} \mathrm{O}_{3}$ present : $x$, therefore gives the figure to be deducted from the uncorrected value for $\mathrm{FeO}$. That this correction is very needful with many of the basic rocks becomes at once evident from the following perhaps extreme example:

Found 2.50 per cent apparent $\mathrm{FeO}$ in a rock containing .13 per cent $\mathrm{V}_{2} \mathrm{O}_{3}$.

Deduct .25 per cent $\mathrm{FeO}$ equivalent in its action on $\mathrm{KMnO}_{4}$ to $.13 \mathrm{~V}_{2} \mathrm{O}_{3}$.

Leaving 2.25 per cent $\mathrm{FeO}$ corrected.

Found 5.00 per cent apparent total iron as $\mathrm{Fe}_{2} \mathrm{O}_{3}$ in the same rock.

Deduct .14 per cent $\mathrm{Fe}_{2} \mathrm{O}_{3}$ corresponding to .13 per cent $\mathrm{V}_{2} \mathrm{O}_{3}$.

Leaving 4.86 per cent corrected total iron as $\mathrm{Fe}_{2} \mathrm{O}_{3}$.

Deduct 2.50 per cent $\mathrm{Fe}_{2} \mathrm{O}_{3}$ equivalent to 2.25 per cent $\mathrm{FeO}$.

Leaving 2.36 per cent $\mathrm{Fe}_{2} \mathrm{O}_{3}$ in the rock.

Failure to correct for the vanadium in both cases would have made the figures for $\mathrm{FeO}$ and $\mathrm{Fe}_{2} \mathrm{O}_{3}$, respectively, 2.50 and 2.22 instead of 2.25 and 2.36 as shown above.

Carbonaceous matter.-As said before (p. 94), matter of organic origin other than graphitic carbon renders the results of the ferrous iron determination altogether unreliable.

\section{UNCERTAINTIES OF THE FERROUS IRON DETERMINATION.}

From the foregoing it is apparent that, despite the utmost care in practical manipulation, the exact estimation of ferrous iron in rocks is one fraught with extraordinary difficulties and uncertainties. Only in absence of decomposable sulphides, and when the amount and condition of vanadium are known, can the result be regarded as above suspicion.

\section{ALKalies.}

THE LAWRENCE SMITH METHOD.

The various methods for getting at the alkalies in insoluble silicates differ more in the mode of attack of the mineral powder and in the immediately subsequent treatment than in the final stages. With very few exceptions, since the early days of the Survey's existence, all alkali determinations have been made by the method of J. Lawrence Smith, ${ }^{1}$ which is far more convenient than and fully as accurate as those in which decomposition is effected by hydrofluoric and sulphuric acids, or by bismuth, lead, or boric oxides. One of its chief advantages is the entire elimination of magnesia at the start.

Decomposition of the powder is effected by heating it with its own

\footnotetext{
1Am. Jour. Sci., 2d series, Vol. L, p. 269,1871; Am. Chemist, Vol. I, 1871; Annalen Chem. und Pharm., Vol. CLIX, p. 82, 1871.
} 
weight of ammontum chloride and eight times as much precipitated calcium carbonate.

The ammonium chloride used must be purified, preferably by sublimation, or made by neutralizing pure ammonia by pure hydrochloric acid, and the calcium carbonate is best obtained from pure calcite by solution and reprecipitation. However obtained, this last is rarely free from alkalies, which must be estimated once for all in a blank test in order to apply a correction. Eight grams of the carbonate will contain usually from 0.0012 to 0.0016 gram of alkali chlorides, almost entirely the sodium salt, but the amount has been brought down to half the above by very long washing. This correction may be admitted at once to be a defect of the method, but it is one easily applied with safety.

The ignition may be made in a covered crucible of ordinary shape and of about 20 to $30 \mathrm{~cm}^{3}$ capacity, heated to dull redness for not more than two-fifths of its height, but the heat has to be kept so low in this case to avoid loss by volatilization that perfect decomposition is not always assured. Hence, to avoid waste of time in very fine grinding, the form of crucible with cap originally advocated by Smith is very much to be preferred, since it permits, when set at an angle through an opening in the side of a fire-clity cylinder, of the application of the

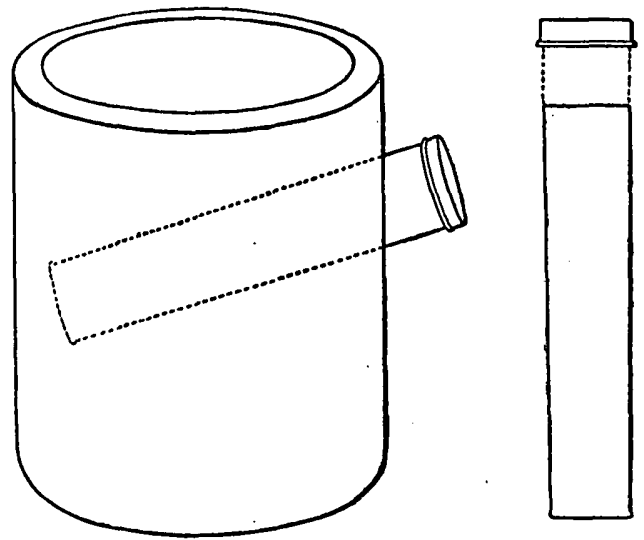

FIG. 14.-The J. Lawrence Smith crucible for alkali determinations. For dimensions see text. .

full heat of two burners, and perfect decomposition invariably resultse without the need of extrayordinary care in grinding. The crucible used in this laboratory (fig. 14) for one-half gram of rock powder and 4 grams calcium carbonate is $8 \mathrm{~cm}$. long, $1.8 \mathrm{~cm}$. wide at the mouth, and 1.5 at the bottom. For double the amounts or more the dimensions are $8 \mathrm{~cm} ., 2.5 \mathrm{~cm}$., and $2.2 \mathrm{~cm}$. The weights are 25 and 40 grams.

Treatment of the mineral powder.-Perfectly satisfactory results are to be obtained with but a half gram of rock powder. This is weighed out, ground down somewhat finer in a large agate mortar, mixed with its own tveight of sublimed ammonium chloride, and the two thoroughly ground together. Then nearly all of 4 grams of calcium carbonate is added and the grinding continued till a thorough mixing has resulted. The contents of the mortir are transferred to the long crucible, the rest of the carbonate being used for rinsing off mortar and pestle. The crucible is then capped and placed in a clay cylinder (fig. 14), or through Bull. $176-7$ 
a hole in a piece of stout asbestos board clamped in a vertical position, and heated for about ten minutes by a low, flat flame placed at considerable distance beneath. As soon as the odor of ammonia is no longer perceptible the nearly full flame of two Bunsen burners is applied, and continued for forty to fifty-minutes. The sintered cake ${ }^{1}$ detaches readily from the crucible as a rule; if not, it is softened up in a few minutes by hot water and digested in a dish until thoroughly disintegrated. It is first washed by decantation, and any lumps are broken up by a pestle, then thrown on the filter and well washed with hot water. The residue should dissolve completely in hydrochloric acid without showing the least trace of unattacked mineral, not even of quartz.

Separation of calcium and sulphuric acid. - All but a trifling amount of the calcium is separated at boiling heat in a large platinum dish by double precipitation by ammonia and ammonium carbonate. The combined filtrates are evaporated to dryness and the ammonium salts are carefully driven off. From the aqueous solution of the residue-but a few cubic centimeters in bulk-the rest of the calcium is thrown out by ammonia and ammonium oxalate, this last being more effective than the carbonate. The filtrate, caught in an untared platinum crucible or small dish, is evaporated to dryness and gently ignited; the residue is moistened with hydrochloric acid to decompose any alkali carbonatc that may have been formed, again evaporated, ignited, and weighed. On solution in water a few tenths of a milligram of fixed residue is invariably left, which should be collected, ignited, and weighed in the same crucible or dish in order to arrive at the weight of the chlorides.

If the rock contains sulphur this will be in part found with the chlorides as sulphate. Therefore, if the sulphur is at all considerable in amount it must be removed by a drop of barium chloride before the final precipitation of the calcium. The excess of barium is removed - by ammonium carbonate and the last of the calcium by ammonium oxalate, as above. If the sulphur is not thus removed there is danger, if not certainty, of the potassium-platinic chloride carrying sodium sulphate. A faint reaction for sulphate can usually be obtained, anyway, if the evaporations have been made on a water bath heated by gas.

Precipitation of potassium.-To the solution of the chlorides in a small porcelain ${ }^{2}$ dish an excess of platinic chloride solution is added. The dilution should be such that when heated on the water bath any precipitate that may form wholly redissolves. Evaporation is then carried on till the residue solidifies on cooling. It is'then drenched

\footnotetext{
${ }_{1}$ To avoid the formation of a melted cake with silicates very high in iron it is advisable to increase the proportion of calcium carbonate.

2 Preferred to platinum becnuse of the possibility, under certain rare and ill-understood conditions, of the formation of an insoluble subchloride of platinum, probably by reaction between the platinum of the dish and that of the salt. (See also Bohn, Zeitschr. für anal. Chemie, Vol. XXXVIII, p. 349, 1899.)
} 
with absolute alcohol ${ }^{1}$ or with that of 80 per cent strength, filtered by decantation through a very small filter and washed by decantation with alcohol of the same strength. The precipitate is not brought onto the filter more than can be avoided. Dish and filter are then dried for a few minutes to remove adhering alcohol, the contents of the former are transferred to a weighed platinum crucible or very small dish, and what still adheres to the porcelain is washed through the filter with hot water into the weighed receptacle. This is now placed on the water bath and afterwards heated to $135^{\circ} \mathrm{C}$. in an air bath. The factor used for reduction of $\mathrm{K}_{2} \mathrm{PtCl}_{6}$ to $2 \mathrm{KCl}$ is 0.307 and of $2 \mathrm{KCl}$ to $\mathrm{K}_{2} \mathrm{O}, 0.632$.

\section{LITHIUM.}

After separation of the potassium platinic chloride, the alcoholic filtrate is evaporated and tested spectroscopically for lithium. This element is almost invariably present, but almost never in amount to warrant quantitative estimation. Should it be so, however, the very excellent Gooch method (summarized below) of separation by amyl alchohol is to be followed, after removal of the platinum by hydrogen gas. $^{2}$ In rock analysis there need be no fear of enough lithium falling with the potassium to cause any concern.

If ammonium carbonate alone has been relied on to separate all calcium (ante, p. 98) the few tenths of a milligram of calcium chloride that escaped precipitation can now be found with the sodium.

GOOCH'S METHOD ${ }^{8}$ FOR SEPARATING LITHIUM.

To the concentrated solution of the chlorides amyl alcohol is added and heat is applied, gently at first, to avoid danger of bumping, until, the water disappearing from solution and the point of ebullition rising and becoming constant for some minutes at a temperature which is approximately that at which the alcohol boils by itself, the chlorides of sodium and potassium are deposited and lithium chloride is dehydrated and taken into solution. At this stage in the operation the liquid is cooled and a drop or two of strong hydrochloric acid added to reconvert traces of lithium hydrate in the deposit, and the boiling continued until the alcohol is again free from water. If the amount of lithium chloride present is small, it will now be found in solution and the chlorides of sodium and potassium, will be in the residue, excepting the traces for which correction will be made subsequently. If, however, the weight of lithium chloride present exceeds 10 or 20 milligrams, it is advisable at this point,

1 Precht (Zeitschr. für anal. Chemie, Vol. XVIII, p. 513, 1879) claims that this is to be preferred to 80 per cent alcohol, especially if evaporation has been carried to dehydration of the sodium salt. Atterberg disputes this final statement and says that 80 per cent alcohol gives better results.

2When haste is not an object, this way of Bunsen's for removing platinum from the chlorides of the alkalies is by far the neatest and most satisfactory. The small flask containing the solution is placed in a water bath and attached to a hydrogen generator. After expelling all nir the flask is closed, without breaking connection with the generator, and left to itself, except for occusional light shaking up, till reduction is accomplished. A more expeditious and very satisfactory reduction is effected by evaporating the solution to dryness with metallic mercury, then herting to expulsion of the excess of mercury and of its chloride (Sonstadt, Jour. Chem. Soc., LXVII, p. 984, 1895), who thus reduces potassium-platinic chloride in order to weigh its platinum.

3 Proc. Am. Acad. Arts Sci., p. 177, 1886; Bull. U. S. Geol. Survey No. 42, p. 73, 1887; Chemical News, Vol. LV, pp. 18, 29, 40,56,78, 1887; Am. Chem. Jour., Vol. IX, p. $33,1887$. 
though not absolutely essential to the attainment of fairly correct results, to decant the liquid from the residue, wash the latter a little with anhydrous amyl alcohol; dissolve in a few drops of water, and repeat the separation by boiling again in amyl alcohol. For washing, amyl alcohol, previously dehydrated by boiling, is to be used, and the filtrates are to be measured apart from the washings. In filtering it is best to make use of the perforated crucible and asbestos felt, and apply gentle pressure. The crucible and residue are ready for the balance after drying for a few minutes directly over a flame turned low. The weight of insoluble chlorides actually obtained in this manner is to be corrected by the addition of $0.00041 \mathrm{gram}$ for every $10 \mathrm{~cm} .^{3}$ of amyl alcohol in the filtrate, exclusive of washings, if the insoluble salt is entirely sodium chloride, 0.00051 gram for every $10 \mathrm{~cm}^{3}$ if potassium chloride constitutes the residue, and if both sodium and potassium chlorides are present, $0.00092 \mathrm{gram}$; but *** the entire correction may in any case be kept within very narrow limits if due care be given to the reduction of the volume of residual alcohol before filtration. The filtrate and washings are evaporated to dryness, treated with sulphuric acid, the excess of the latter driven off, and the residue ignited to fusion and weighed. From the weight thus found the subtraction of $0.00050 \mathrm{gram}$ is to be be made if sodium chloride constitutes the precipitate, 0.00059 gram if potassium chloride alone is present in the residue, and $0.00109 \mathrm{gram}$ if both these chlorides are present, for every $10 \mathrm{~cm} .^{3}$ of filtrate, exclusive of washings.

Amyl alcohol is not costly, the manipulations of the process are easy, and the only objectionable feature-the development of the fumes of amyl alcohol-is one which is insignificant when good ventilation is available.

The process has been used for some months frequently and successfully, by others as well as by myself, for the estimation of lithium in waters and minerals.

\section{SEPARATION OF ALKALIES BY OTHER METHODS.}

When, as may happen in rare instances, it is necessary to cstimate alkalies in the main portion after elimination of silica, alumina, lime, etc., in one of the usual ways, the question of a suitable method for separating magnesium becomes important.

The mercuric oxide method.-The old barium-hydroxide method is not to be recommended. The mercuric oxide method of Zimmermann, whereby the magnesia is precipitated from solution of the chlorides by moist, freshly precipitated, and alkali-free mercuric oxide, can give satisfactory results. The oxide is added in excess to the solution in a platinum crucible and evaporated to dryness. Then the mercuric chloride and most or all of the excess of oxide are expelled by cautious heating. On leaching with water the magnesia remains on the filter. With more than 1 per cent of magnesia the operation must be repeated (Dittrich).

The ammonium carbonate method.-Lately the once favored method of precipitating the magnesium by neutral ammonium carbonate in concentrated solution has been again recommended. ${ }^{1}$ The magnesium solution must be as strongly concentrated as possible, and a great excess of ammonium carbonate solution must be used. A voluminous

\footnotetext{
'Wülfing: Ber. deutsch. chem. Gesell., Vol. XXXII, p. 2214, 1899. The neutral carbonafe is prepared by dissolving 230 grams of ammonium carbonate in 180 cubic centimeters of ammonia of 0.92 specific gravity and enough water to make 1 liter.
} 
precipitate forms, which dissolves on vigorous stirring if enough of the precipitant was used. After a time a crystalline precipitate fallsa double carbonate of magnesium and ammonium-which is insoluble in a concentrated solution of ammonium carbonate. Allow to stand for six to twenty-four hours. Wash with the concentrated ammonium carbonate solution. It is probably no exercise of undue caution to redissolve and reprecipitate, to make sure of getting all alkali in the filtrate.

The amyl alcohol method.-Under certain circumstances, notably absence of lithium, the method of Gooch developed by Riggs ${ }^{1}$ may be satisfactory. It is similar to that of Gooch for separating lithium from sodium and potassium chlorides by amyl alcohol, and involves the same solubility corrections for the alkali chlorides above noted (p. 100) in the description of Gooch's method. Riggs's summary is as follows:

Evaporate the solution nearly or quite to dryness. Dissolve the residue in as little water as possible and add a few drops of hydrochloric acid. Then add 30-40 cubic centimeters of amyl alcohol and expel the water by bringing the alcohol to boiling. Continue the boiling until the volume of the solution is reduced to 10 cubic centimeters, or even considerably less. In filtering, it is of great advantage to use a perforated crucible and an asbestos felt and to filter under pressure. In case the total chlorides exceed 0.2 grams it may be advisable to decant the liquid, wash the residue, redissolve, and repeat the precipitation. If this be not done, the precipitate should be redissolved with the least possible quantity of water, a few drops of hydrychloric acid added, and the precipitation repeated in the original solution. The filtrate is transferred to a weighed platinum dish and evaporated. Water is added before the alcohol has been expelled, and the evaporation continued. The residue is dissolved in water. Sulphuric acid is added in slight excess. This solution is evaporated to dryness, the residne ignited and weighed, and the treatment with sulphuric acid is repeated. The residue of insoluble chlorides may be transferred to the weighed perforated crucible and dried at a temperature below their melting points, or it may be dissolved and the solution transferred to a weighed platinum dish, evaporated, and the residue dried as above and weighed.

As with the Gooch method for lithium, the numerous test results are good.

\section{CARBON DIOXIDE, CARBON.}

For this estimation an apparatus (fig. 15) permanently set up is used, of which several forms have been described by different writers. The rock powder is boiled with dilute hydrochloric acid in a small Erlenmeyer flask, attached to an upward-inclined condenser, whence, after passing through a compact arrangement of drying tubes-first, one of calcium chloride, then one of anhydrous copper sulphate to retain hydrogen sulphide from decomposable sulphides and any hydrochloric acid that may pass over, and finally a second calcium chloride tube-the carbon dioxide is retained by absorption tubes filled with soda-lime followed

${ }^{1}$ Am. Jour. Sci., 3d series, Vol. XLIV, p. 103, 1892. 
by calcium chloride. Of course arrangement is made for a current of $\mathrm{CO}_{2}$-free air with which to sweep out the apparatus before and after the experiment, and for a slow current during its continuance. The

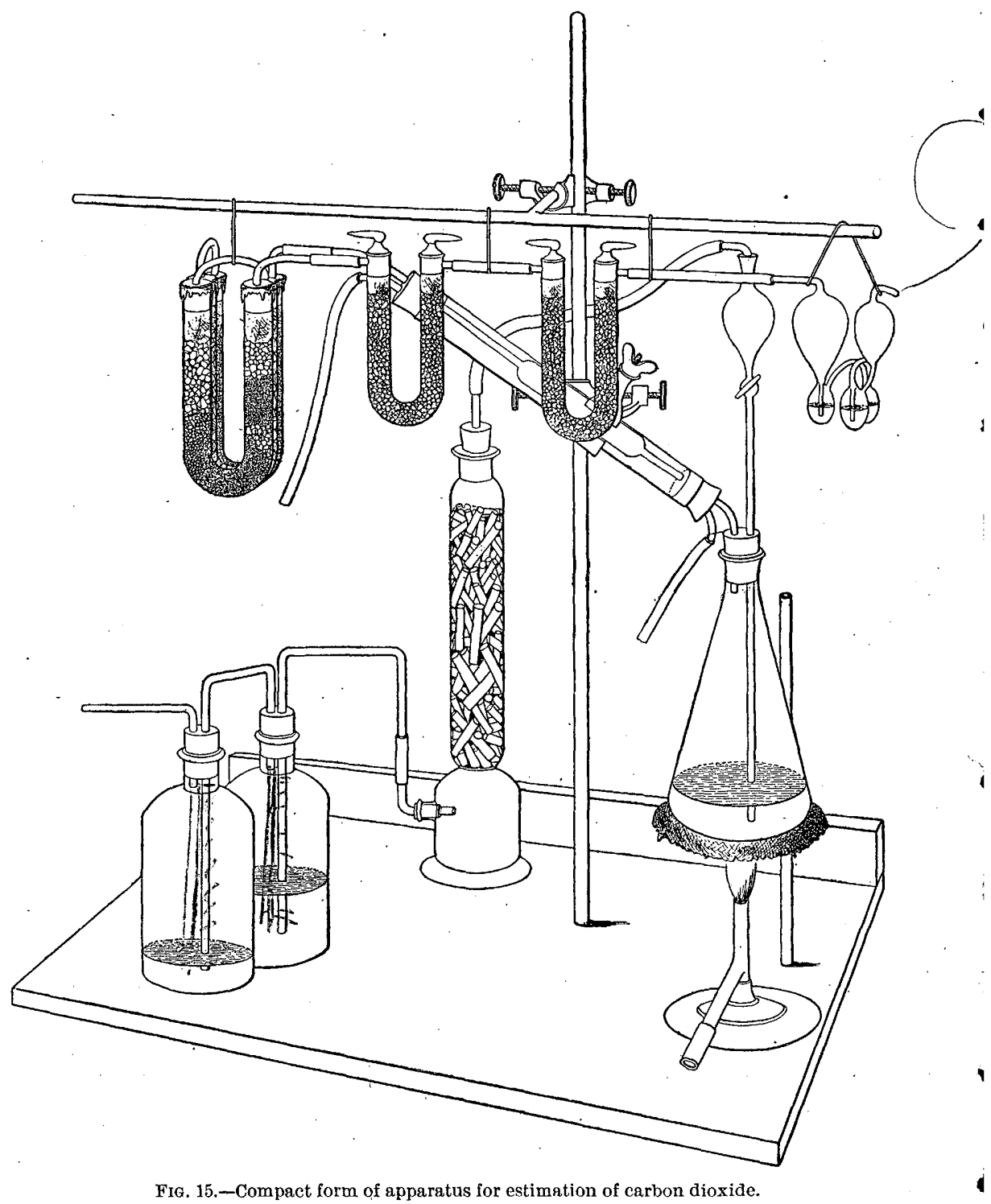

results are very accurate and the determination can be quickly carried out.

In the preliminary qualitative test for carbon dioxide, it must be remembered that while calcite gives off its carbon dioxide on treatment with cold acid, dolomite and siderite do not, and hence warming 
should not be omitted; otherwise, a few tenths per cent of carbon dioxide can very well be overlooked. Moreover, the powder should first be stirred up with a little hot water, to remove all entangled air which might otherwise be mistaken for carbon dioxide.

It has been already shown under Water (p. 45) how, in case of need, the determination of carbon dioxide can be combined with that of water by fusion with lead chromate or potassium chromate. This latter method must always be resorted to when the carbon of graphite or carbonaceous matter has to be estimated. If carbonates are present at the same time the result of the test includes the carbon from both sources, and a separate determination by the wet way of that of the carbonates is necessary.

\section{CHLORINE.}

To make sure of getting all the chlorine, it is best to fuse with chlorine-free sodium-potassium carbonate, or even sodium carbonate alone, first over the full burner, then for a moment or two over the blast, leach with water, acidify with nitric acid, and precipitate by silver nitrate without preliminary separation of silica. If 1 gram of material has been used no precipitation of silica need be feared on acidifying or on standing.

In many cases it is quite sufficient to attack the powder by chlorinefree hydrofluoric acid and a little nitric acid, with occasional stirring, and after filtering through paper fitted into a large platinum cone or rubber funnel, to throw down the chlorine by silver nitrate. The presence of nitric acid is necessary, since otherwise ferrous fluoride reduces silver nitrate with deposition of crystallized silver. When coagulated by heating and stirring, the precipitate is collected on the filter, washed, dissolved by a little ammonia, and reprecipitated by nitric acid, when it can be collected in a Gooch crucible and weighed, or, if very small in quantity, on a small paper filter, which is then dried, wound up in a tared platinum wire, and carefully ignited. The increased weight of the wire is due to the metallic silver of the chloride which has alloyed with it.

\section{FLUORINE.}

Fluorine can only be estimated by the method of Rose, care being taken to use sodium-potassium carbonate as a flux, and to avoid use of the blast if possible. For minerals rich in fluorine and low in silica it may be necessary to add pure silica before the fusion in order to effect complete decomposition of the fluoride just as with the alkalineearth phosphates. To the hot aqueous extract several grams of ammonium carbonate are added and more on cooling. After twelve 
hours the solution is filtered, ammonium carbonate is expelled, and an ammoniacal solution of zinc oxide added, whereupon evaporation is carried on till the odor of ammonia is entirely gone. After filtering, add nitric acid in insufficient quantity to fully neutralize.

The use of ammonium nitrate or chloride, instead of carbonate, for throwing out the silica and alumina is not to be recommended, because of loss of fluorine on evaporation (Rose). If the rocks are very basic it may happen that the amount of silica in the alkaline solution is so small that ammonium carbonate may be dispensed with and the anmoniacal zinc oxide solution added at once.

By whatever modification of the method the silica and alumina may have been separated, the alkali carbonate must be converted into nitrate and not chloride if phosphorus or chromium, or both, are present. "To remove the chromium and the last of the phosphorus, silver nitrate in excess is added to the solution containing still enough alkali carbonate to cause a copious precipitate of silver carbonate, which shall take up the acid set free and thus insure a neutral solution and consequent complete precipitation of phosphorus and chromium. After heating and filtering, the excess of silver is to be removed by sodium or potassium chloride, and sodium carbonate is to be added, in order to furnish by addition of calcium chloride in excess to the hot solution a sufficient admixture of calcium carbonate with the fluoride. At this stage there must be no ammoniacal salts in solution; otherwise calcium fluoride may be held up.

The next operation is perhaps best conducted as recommended by Penfield and Minor. ${ }^{1}$ To the gently ignited precipitate of carbonate and fluoride of calcium at most $1-2 \mathrm{~cm} .{ }^{3}$ of acetic acid are added in the crucible, which is then placed for a time on the water bath and afterwards its contents are evaporated to dryness. They are then taken up: with hot water, the solution is decanted through a small filter, likewise the washings. The filter is burned in the same crucible, more acetic acid is added and the various operations are repeated until all calcium oxide and carbonate have been extracted. The above authors find that if a great excess of acetic acid is used at the start, the results are low. While their experiments related to the determination of fluorine in a mineral rich in that element-topaz-their precautions are probably not needless with the small amounts of fluorine met with in rocks.

The well-washed and gently ignited calcium fluoride finally obtained in the course of this method should be converted to sulphate as a check upon its.purity, and at the same time as a qualitative test to ascertain if it really is calcium fluoride by the characteristic odor of the gas given off. - Should fluorine be found, and the weight of sulphate not correspond to that of the fluoride, the former should be dissolved in 
hot nitric acid and tested for phosphorus by ammonium molybdate solution. If phosphate is absent the impurity may have been silica or calcium silicate- which of these it would be difficult to decide. In the former case the fluorine might be safely deduced from that of the sulphate, but not in the latter. If the rock were rich in sulphur it might happen that calcium sulphate would be thrown down with the fluoride, but this should be removed by thorough washing. If not, and it were certainly the only impurity present, the fluorine could be calculated, after conversion of the fluoride into sulphate, by the formula:

$\mathrm{CaSO}_{4}-\mathrm{CaF}_{2}: 2 \mathrm{~F}::$ Diff. between impure $\mathrm{CaSO}_{4}$ and $\mathrm{CaF}_{2}: x$.

It is an exceptional case when there is exact agreement between the weight of fluoride and sulphate, and with the small amounts usually met in rocks the error may be an appreciable one in percentage of fluorine, though of no great significance otherwise.

There is no qualitative test which will reveal with certainty the presence of fluorine in rocks. Heating the powder before the blowpipe with sodium metaphosphate on a piece of curved platinum foil inserted into one end of a glass tube, or in a bulb tube, is not to be relied on in all cases. 'While as little as one-tenth of 1 per' cent of fluorine can sometimes be thus detected with ease, much larger amounts in another class of rocks may fail to show.

\section{SULPHÜR.}

Before proceeding to the estimation of sulphur, its condition, if present, should be ascertained.

Evolution of hydrogen sulphide on boiling with hydrochloric acid is evidence of a soluble sulphide, usually pyrrhotite, but possibly lazurite. Extraction of magnetic particles reacting for sulphur shows pyrrhotite to have been in part at least the source of the hydrogen sulphide. A reaction for sulphuric acid in the filtered solution indicates a soluble sulphate, usually noselite or hauynite. If the residue, when well washed and treated with aqua regia or hydrochloric acid and bromine, gives more sulphuric acid, the probable presence of pyrite is shown. Should this solution likewise sbow arsenic, the sulphide may be arsenopyrite, which, however, is of very rare occurrence in igneous rocks, if, indeed, it is ever found there.

For the quantitative extraction of the sulphur of soluble sulphates, simple boiling with hydrochloric acid suffices, which should be done in an atmosphere of carbonic acid if pyrites or other oxidizable sulphides are present, and should be finished as quickly as possible in order to minimize the error resulting from oxidation to sulphuric acid of the sulphur: of sulphides, if present, by any ferric salts that may have been dissolved. 
The sulphur of sulphides may sometimes be correctly determıned by extraction with aqua regia or some other powerful oxidizer, but not always; so that it is better by far to fuse with. sulphur-free sodium carbonate and a little niter over the Bunsen burner, and for a few moments over the blast, fitting the crucible into a hole in asbestos board (Lunge) to prevent access of sulphur from the flame. After thorough disintegration of the fusion in water, to which a drop or two of alcohol has been added for the purpose of reducing manganese, the solution is filtered and the residue washed with a dilute solution of sodium carbonate. In the filtrate $\left(100-250 \mathrm{~cm}^{3}\right.$ in bulk) the sulphur is precipitated at boiling heat by barium chloride in excess after slightly acidifying by hydrochloric acid. . Evaporation to dryness first with acid, in order to eliminate silica, is needless, for in the above bulk of solution there will almost never be the least separation of silica with the barium sulphate. It is well that this is so, for evaporation on the water bath heated by gas to remove silica would in many cases involve an error fully equal to the sulphur present by contamination from the sulphur of the gas burned.

Owing to the small amount of sulphur in rocks, special purification of the barium sulphate obtained is hardly ever needful, especially as it has been precipitated in absence of iron. Should there be fear of a trace of silica being present, it can be removed by a drop of hydrofluoric and sulphuric acids before weighing the barium sulphate.

This, of course, gives the total sulphur in the rock. If soluble sulphates and sulphides as well as insoluble sulphates and sulphides are present together, the sulphur of the first is found in solution after extraction by hydrochloric acid in a carbon-dioxide atmosphere, and that of the decomposable sulphides by collecting the hydrogen sulphide evolved. ${ }^{1}$ In the residue the sulphur of the insoluble sulphides can be estimated, or from the total sulphur found in another portion its amount can be calculated. The error involved in the above estimation of the sulphur of soluble sulphides, due to the possible reducing effect of hydrogen sulphide on ferric salts, is probably negligible. Most of the hydrogen sulphide would be expelled before any such action could take place and probably before the ferric salts were largely attacked, but of course the small proportion of sulphur set free as such from pyrrhotite would escape estimation and introduce further uncertainty. In general, it would be safe enough to assume the composition $\mathrm{Fe}_{7} \mathrm{~S}_{8}$ for pyrrhotite. However carefully all these separate determinations may be carried out, the final figures for ferrous and ferric oxides can hardly be regarded as more than approximations when much sulphide is present. (See pp. 94-95.)

${ }^{1}$ With pyrrhotite a small fraction of its sulphur-one-eighth if the formula $\mathrm{Fe}_{7} \mathrm{~S}_{8}$ is adopted-is liberated as free sulphur and not as hydrogen sulphide. 
To the best of the writer's belief, it has never been found necessary in this laboratory to estimate boron in a silicate rock. Should the determination be required, since most silico-borates are insoluble minerals, it would probably be necessary to fuse with sodium carbonate, extract with water, faintly acidify with nitric or acetic acid, expel the boron by distillation with methyl alcohol, and collect the horic ether in a suitable manner. For simple borates, artificial or native, this method, ${ }^{1}$ first devised by Rosenbladt and Gooch independently, gives entire satisfaction when all needful precautions are carefully observed, but its application to boro-silicates yet needs investigation, in view of the as yet unexplained very discordant results obtained some years ago by J. E. Whitfield in this laboratory on the mineral warwickite, a boro-titanate of magnesium and iron.

It is also quite possible that the accurate estimation of but a few milligrams or even less of boric oxide by the use of a large excess of lime as a retainer would not be feasible. Fluorine would have to be first removed by calcium nitrate or acetate before freeing the boron.

\section{NITROGEN.}

Nitrogen has been found in igneous rocks or the minerals occurring in them by several observers. Thus, H. Rose ${ }^{2}$ says that pitchstone gives off ammoniacal water on heating; Silvestri ${ }^{3}$ mentions a nitride of iron in lavas from Etna; Sandberger finds ammonium carbonate to be given off from certain rocks of Pribram; the writer has shown nitrogen to exist in uraninite; Ramsay and others have noted it in traces with or without helium, etc., in numerous minerals; and later Erdmann ${ }^{4}$ found it to be given off as ammonia on treating various minerals of "ancient igneous rocks" with a caustic alkali. Luedeking also found ammonium sulphate in a barite from Missouri, the presence of which the writer was able to confirm.

It has been noted in this laboratory on three separate occasions, when series of ores, roofing slates, and eruptive rocks were analyzed, that

\footnotetext{
1 Rosenbladt (Zeitschr. für anal. Chemie, Vol. XXVI, p. 21, 1887) used magnesia for binding the boron, while Gooch (Proc. Am. Acad. Arts and Sci, p. 167, 1886: Bull: U. S. Geol. Survey No. 42, p. 64: Chemical News, Vol. LV, p. 7, 1887) preferred lime, as more active and reliable. Gooch and Jones have later (Am. Journ. Sci., 4th series, Vol. VII, p. 34, 1899; Chemical News, Vol. LXXIX, pp. 99, 111, 1899) upheld the use of lime and proposed, as a convenient though perhaps not quite so perfect substitute, sodium tungstate containing an excess of tungstic oxide. In this article they likewise indicate the precautions now used to insure complete collection and retention of the boron.

For a useful modification in the way of collecting the boric ether in ammonia before bringing in contact with the lime, see Penfield and Sperry (Am. Journ. Sci., 3d series, Vol. XXXIV, p. 222, 1887); also Moissan (Comptes Rendues, Vol. CXVI, p. 1087, 1893, and Bull. Soc. Chim., Vol. XII, p. 955, 1894), who modifies the Gooch distilling apparatus in certain respects.

2 Quantitative Analyse, p. 673 . Finkener edition.

${ }^{3}$ Gazz. chim. ital., Vol. V, p. $303,1875$.

1 Ber. Deutsch. chem. Gesell., Vol. XXIX, p. 1710, 1896.
} 
ammonia, either in the form of chloride or sulphate, or even as free ammonia, was given off on heating. Its appearance was not limited to one or a few specimens of a series, but seemed to be characteristic of all, and to be afforded by the unbroken rock as well as by the powdered sample. The precise conditions under which the specimens were collected not being known, it is impossible to affirm positively that the ammonia may not have been due to recent organic contamination of some sort, especially in the case of the slates, but it is believed that a more critical collection of material will not alter the general result. Its amount was sometimes readily determinable by Nesslerization, being as high as 0.04 per cent in some slates. Carbonaceous organic matter was absent from most of these, but doubtless existed in them in their early history. In their case the ammonia was, in part at least, evolved as such, imparting a strong alkaline reaction to the water in the upper part of the tube. The presence of sulphides, fluorides, or chlorides in the rock might cause the ammonia to appear as a sublimate of sulphate, fluoride, or chloride. Speculation on this matter would be altogether premature, but attention is called to it in the hope that other observers may be led to look for and investigate similar appearances. It should be borne in mind that the nitrogen present would not necessarily appear as ammonia or ammonium salts, since it might be given off in the elemental condition, as with the gases obtained from uraninite.

\section{SPECIAL OPERATIONS.}

The problem often presents itself of ascertaining the composition of that portion of a rock powder which is soluble in special reagents or in a reagent of a particular concentration. No precise directions can be formulated to meet such cases. The procedure must vary with the character of the constituents of the rock and with the object which it is sought to attain, and only in exceptional cases can a separation of this kind be sharp. Much depends on the degree of fineness of the powder and on the length of time it is exposed to the action of the reagent.

\section{DETECTION OF NEPHEIINE IN PRESENCE OF OLIVINE.}

For confirmation of the microscopic diagnosis, Prof L. V. Pirsson ${ }^{1}$ has indicated a means of detecting nepheline in presence of olivine, as in nepheline basalts, based on the very ready solubility of nepheline, as compared with olivine, when boiled for but one minute with a sufficiency of very dilute nitric acid (1:40). Gelatinization of the filtrate on evaporation is taken as evidence of the presence of nepheline. If olivine 
is present in quantity, however, this test must not be accepted at once as final, for some, if not a!l, olivines are much more soluble in nitric acid of the above strength than Professor Pirsson was led to believe from his original tests. If, therefore, on evaporation of the filtrate, much iron is indicated, the gelatinization may well be due to olivine alone or in part, and then the quantitative relation of silica to iron plus magnesium should be ascertained. It must also be borne in mind that any other very soluble silicates present will be more or less affected, and that apatite is largely or wholly dissolved. It is possible that still more dilute nitric, or perhaps some other, acid may exert a slighter solvent action on olivine without being appreciably less effective in dissolving nepheline, etc. In combination with a quantitative analysis of the extract the method is perhaps susceptible of a wider application than the particular case for which it was first used. It is well worth further study.

\section{ESTIMATION OF SOLUBLE SILICA.}

Very often in treatment by acids silica is separated in gelatinous or granular form mixed with the unattacked minerals, and it becomes necessary to remove or estimate this silica, or else to discriminate between soluble and insoluble silica already existing together. Usually a boiling solution of sodium carbonate has been employed for this purpose, though the caustic alkalies have found advocates.

Lunge \& Millberg ${ }^{1}$ have lately conclusively shown that quartz is not nearly so insoluble in solutions of the caustic alkalies as has been supposed, but that given a sufficient degree of subdivision it can be brought wholly into solution; that it is impossible to secure correct separation of quartz and opaline silica by the use of either caustic or carbonated alkalies; and that digestion on the water bath for $15 \mathrm{~min}$ utes with 5 per cent solution of sodium carbonate is the only way to secure exact separation of unignited precipitated silica from quartz, and then only when the finest flour has been removed by levigation. The authors say:

If, however, no more of such flour is present than is produced in the ordinary operations of powdering and sifting through cloth of the finest mesh, the error arising from the above mentioned treatment is so slight that it can generally be neglected; it reaches 0.1 to at the most 0.2 per cent of the total silica, by which amount the quartz will appear too low, the amorphous silica too high.

The above authors also show, however, that the solvent action of the caustic alkalies on quartz becomes very apparent only when the material has been reduced to such an utterly impalpable degree of fineness as is practically never reached in the preparation of samples for rock analysis. For this reason I have no hesitation in recommending the employment of a dilute solution of sodium hydroxide when 
the silica separated by acid from one of several mineral constituents of a rock is to be estimated. Even when dilution is conssderable, solution is almost immediate, and as soon as this is accomplishedthe point being known by the change in appearance of the residuethe solution should be diluted with cold water and filtered at once. The difficulty met with in filtration may often be overcome by faintly acidifying, which has the added advantage of at once arresting any further action of the alkali. If the dilution is sufficient no separation of silica results from so doing. Very dilute acid should also be used for washing. Lunge, when using sodium carbonate, washes with hot carbonate solution to which alcohol has been added, thus obtaining clear filtrates.

\section{ESTIMATION OF MINUTE TRACES OF CERTAIN CONSTIT-} UENTS.

If, as sometimes may happen, the problem is presented of examining rocks for traces of gold, silver, and other elements which are not ordinarily looked for, as in Sandberger's investigations bearing on the origin of the metalliferous contents of veins, large weights of material must be taken, up to 50 grams or more. This involves the use, also, of large quantities of reagents, the purity of which must then be looked to with the utmost care. Special directions to meet such cases can not now be given, nor even a complete reference list of the scanty and scattered literature on this subject. Sandberger's own writings deal but little with its analytical side, and from its inaccessibility in the Washington libraries the writer is as yet unacquainted with the report by Von Foullon "Ueber den Gang und die Ausführung der chemischen Untersuchung," "following Sandberger's own paper in the general report "Untersuchungen der Nebengesteine der Pribramer Günge." ${ }^{2}$ The present writer has published a few data as to gold, silver, lead, zinc, etc., ${ }^{3}$ in Mr. S. F. Emmons's report on "The Geology and Mining Industry of Leadville;" and Mr. J. S. Curtis, ${ }^{4}$ in his report on "The Silver-Lead Deposits of Eureka, Nevada," has given his method of assaying rocks for traces of gold and silver.

To Prof. F. W. Clarke and Dr. H. N. Stokes the writer's thanks are due for friendly suggestions in the preparation of the foregoing work.

\footnotetext{
JJahrbuch der Bergakademie, Leoben u. Pribram, 1887, p. 363.

2From Sindberger's report it appears that the rocks were treated successively with water, acetic ucid, boiling dilute hydrochloric acid for two days, and finally hydrofluoric acid, the several extracts and final residue of fluorides (and pyrite) being separately examined for heavy metals. The products of distillation were also examined. A striking fact observed in all cases was the complete insolubility of the pyrite, even after the severe treatment mentioned. This speaks strongly in favor of the correctness of ferrous iron estimations in silicates by the hydrofluoric and sulphuric acid method when pyrite is present unaccompanied by other sulphides. (See p.95.)

3 Mon. U. S. Geol. Survey, Vol. XII, Appendix B, pp. 592-596, 1886.

${ }^{4}$ Mon. U. S. Geol. Survey, Vol. VII, pp. 120-138, 1884.
} 


\section{N D E X.}

Alkalies, contamination of, by traces of calcium .................... 99

determination of . . . . . . . . . . . . . 96-101

Aluminum, direct method for determin-

$$
\text { ing }
$$

$59-60$

ignition of precipitate of........... 56

indirect method for determining . .... 54-57

methods of determining ............ 54-60

modes of precipitation of . ........... 55-56

Ammonia in rocks, occurrence of ......... 108

Analyses, ingredients sought in.......... 20

limit of allowable error in........... 24

mode of erushing or grinding rock for . 31-32

mode of statement of .............. 21-22

number of portions of powder needed

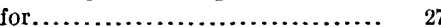

quantity of rock needed for......... . 31, 32

tables showing errors arising from incomplete.

16,17

time needed for making . . . . . . . . . . 22-23

unadvisability of qualitative.......... 26

weight of ground sample needed for.. 32

weights of portions of powder needed for

Austin and Gooch, cited . ...........65,78

Bailey, G. H., cited ................. 75, 76

Barium, contamination of magnesium phosphate by..................

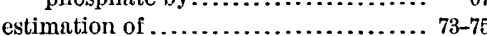

ignited silica free froni.............. 53

percentage of rock content of........ 17-18

preliminary procedure when material is scanty .........................

recovery from alumina precipitate of possible amounts of ............. $66-57$

separation of strontium from .......... 63-64

Basic acetate method of precipitating aluminum, iron, etc ............... 55-50

Baskerville, C., method of separating titanium from iron and aluminum devised by

method of separating zirconium devised by $\ldots . . \ldots \ldots \ldots \ldots \ldots \ldots \ldots$

Baubigny, $\mathrm{H}$., cited.

Beuteln" in preparing rock samp̉le, objections to

Blair, A. A., platinum-tipped crucible tongs devised by .............................

Bohn, C., cited ................ 98 (footnote)

Boric-oxide method for decomposing silicates, description of ............49-50

possible objections to............... $\quad 50$

Boron, estimation of.................. 107

rare occurrence of
Broockmann, K., cited................. 66

Browning, P. E., cited ................. 63

Brühl and Friedheim, cited $\ldots \ldots \ldots \ldots \ldots 61,80$

Brush, G. J., method of water determination devised by ..................... 38

Bunsen, R., cited.............. 99 (footnote)

Calcium, contamination of, in weighed alkali chlorides

contamination of magnesium phosphate by ........................ 67

determination of $\ldots \ldots \ldots \ldots \ldots \ldots \ldots \ldots .62-64$

separation of strontium from ......... 63

Campbell and Hess, cited ................ 60

Carbon, determination of ............. 101-103

Curbon dioxide, determination of....... 101-103 figure showing apparatus for estimution of $\ldots \ldots \ldots \ldots \ldots \ldots \ldots \ldots \ldots . . . \ldots \ldots$

Carbonaceous matter, determination of ferrous iron as affected by .......... 94,96

Carnot, A., cited ...................... 58

Cathrein, A., cited........... 59, 71 (footnote)

Cerium, determination of ............. 77 rare occurrence of $\ldots \ldots \ldots \ldots \ldots \ldots \ldots .21$

Chatard, T. M., cited ................... 79 description and figure of drying oven devised by ..................... 36-37 Gooch gravimetric method of estimating titanium as modified by....... 71-72 method of water determination devised

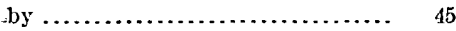

Chlorine, determination of............ 103

Chromium, determination of......... 79-82,83 preliminary procedure when material

is scanty . ...................... 79

tubles showing results of tests for . . . . 86,87

Claassen, E., eited ..................... 83

Clarke, F. W., acknowledgments to ...... 110

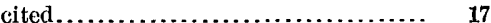

Cloedt and Jamnasch, cited.............61,80

Cobalt, determination of . ............ 61-62

Colorimetric apparatus for estimating titanium, descriptions and figures of ... 70-71

Colorimetic method for determining chromium....................... 80-81

comparison of gravimetric method with $\ldots \ldots \ldots \ldots \ldots \ldots \ldots \ldots \ldots \ldots . . \ldots 1-82$

Columbium, rare occurrence of .......... 21

Cooke, J. P., cited........................ 92

figure of apparatus for determining ferrous iron devised by .......... 93 Copper, determination of . ............ 61-62 Crucible tongs, description and figure of... 23 Crushing rock, mode of ............... 31

Curtis, J. S., cited .................... 110 
Page.

Czudnowicz, C., cited......................
Davis, J. Thomas, method of separating zirconium devised by

Dittrich, M., cited

Doelter, C., modification in method devised

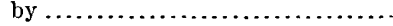

Drying oven for water determinations, description and figure of ............. $36-37$

Drying temperatures in moisture determinations.

Dunnington, F. P., eited .................. Eakins, L. G., pycnometer method of .......

Earths, rare, cautions regarding, in basic acetate method ........... 57 (footuote)

estimation of ......................... 77-78

Erdimann, H., cited .................... 107

Evaporation, description and figure of radiator for ......................... $23-24$

Feidspars, error in calculations of, based on incomplete analyses...............

Ferric iron, determination of............. partial reduction of, by fuulty ignition.. 38, 56

Ferrous iron, determination of............ 88-96 figure showing Cooke's apparatus for determining. uncertainties in estimation of...........

Fischer, T., cited..........................

Fluorine, determination of............. 103-105 qualitative detection of .............. 105

Foullon, H. von, cited ................. $\quad 110$

Franz and Streit, cited.................. 76 method of separating zirconium devised

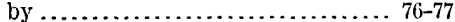

Fresenius, R., cited............ 62 (footnote), 63 Friedel, C., cited on water determinations.. $\quad 35$ Friedheim and Brühl, eited ............. 61, 80 Genth and Penfield, cited on precipitation of aluminum, iron, etc., by ammonia.

Gibbs, Wolcott, cited.

Gilbert, J.P. uited on silicn determination. $52-5$

Glucinum, rare occurrence of .............

Gooch, F.A., amyl-alcohol method of separating lithium devised by ......... 101

apparatus devised by................. 20 cited ..................6, 60,107 (footnote) gravimetric method of estimating titanium devised by ................ $71-73$ method of estimating boron devised by. $\quad 107$ methods of separating lithium devised by $\ldots \ldots \ldots \ldots \ldots \ldots \ldots \ldots \ldots \ldots . . .299-100$ method of water determination devised by ............................. 41-45 Gooch and Austin, cited............... 65,78 Gooch and Jones, cited........... 107 (footnote) Gravimetric method for determining chromium................. 80

comparison of colorimetric method with $81-82$

Grinding rock, mode of ................. 31-32

Harper and Penfield, cited on washing of precipitated alumına..............

Hayes, A. A., cited ..................... 18

Heidenreich and Jannasch, boric-oxide method of decomposing silicates devised by..................... 49-50

Hess and Campbell, cited ................. 60 Hillebrand, W. F., cited..........19, 63 (footnote), $68,75,80$ (footnote), 82,91 (footnote), 110
Page.

Hillebrand and Ransome, cited... ss (footnote) Hillebrand and Stokes, cited...... 90 (footnote) Hydrofluoric acid, cautions concerning quality of...$\ldots \ldots \ldots \ldots \ldots \ldots \ldots \ldots \ldots$

Hydrofluoric-acid method of determining ferrous iron.................. 92-94

Hydrogen peroxide, impurities of ......... 25

Hydrogen sulphide, metals precipitable by . . 54

Hygroscopic water, importance of determining .......................... $35-36$

modes of determining............... 32-37.

Iron, estimation of ................... 57-59.

ferrous, estimation of............... 88-96

ignition of precipitate of............. 56

methods of determining............. 54-60

preliminary procedure when material is scanty .........................

solution by sodium carbonate fusion of traces of . ...................... 58-59

Jannasch, P., methods of water determination devised by ................ 45-47

modes of silica determination devised by ........................... 48-50

Jannasch and cloedt, cited .............6 61, 80

Jannasch and Heidenreich, boric-oxide method of decomposing silicates devised by .................... 49-50

Jannaseh and Weber, boric-oxide method for decomposing silicates devised

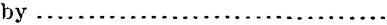

use of oxygen blast in treatment of refractory silicates by the boric-oxide method ...

Jones and Gooch, cited ...........107 (footnote)

Knop, A., cited ................. 57 (footnote)

Koninck, L. L. de, cited ................... 66

Koningh, L. de, colorimetric method of determining chromium devised by..

Krauch, C., cited on tests for purity of chemical reagents.................

Lindemann; Otto, cited .................. 88

Lithium, determination of ............. 99-100

Luedeking, C., cited ...................... 107

Lunge, G., cited ......................... 106

Lunge and Millberg, cited ............... 109

Magnesium, collection and ignition of precipitated phosphate of..............

- contamination by barium of precipitated phosphate of ................

contamination by calcium of precipitated phosphate of ................ 67

determination of ................... $64-67$

separation of calcium and strontium from ........................... 62

Manasse, 0. cited................ 84 (footnote)

Manganese, determination of ............ 60-61

Mar, F. W., cited ...................... $\quad 75$

Mastbaum, Hugo, cited................. 66

Metals precipitable by hydrogen sulphide. $\quad 54$

Microcosmic salt, cautions concerning quality of ............................ 25

Millberg and Lunge, cited............... 109 Mitchell and Wells, cited ......... 57 (footnote) Mitscherlich method for determining ferrous iron, defects of ......... $88-89,89-90$

modification of ..................... 90-92 Moissan, H., cited ............... 107 (footnote) 
rage.

Moisture, modes of determiningr......... 32-37

Molybdenum, distribution of .......... 18, 82 determination of $\ldots \ldots \ldots \ldots \ldots \ldots \ldots . .82-84$ precipitation of, by hydrogen sulphide 84 (footnote) qualitative test for

Nepheline, detection of............... 108-109

Neubatier, Hugo, cited.............. 64, 65, 66

Nickel, determination of .............. 61-62

Nitrogen, estimation of............... 107-108

Oven for water determination, description and figure of $. . . \ldots \ldots \ldots \ldots \ldots \ldots . . . . .$.

Penfield, S. I., cited.

methods of water determination used by ........................ 38-41

mode of determining specific gravity recommended by.

Penfield and Genth, cited on precipitation of aluminum, iron, etc., by ammonia.

Penfield and Farper, cited on washing of precipitated alumina..............

Penfield and Minor, cited................ 104

Penfield and sperry, cited ....... 107 (footmote)

Phosphoric und titanic oxides, combined average of, in rocks of earth's crust.

in ulumina precipitate, peculiar occurrence of ............... 57 (footnote)

Phosphorus, determination of .......... 78-79

Pirsson, L. V., arrangement of analyses proposed by ........................ cited on detection of nepheline ........

I'latinum, presence of, in filtrates from silica removal of from filtrates... 54,57 (footnote)

P'latinum crucibles, loss in weight of, on blusting ............... 53 (footnote)

Potassium bisulphate, cautions concerning mode of preparation of.............

Pratt, J. F., modification in method for determining ferrous iron devised by..

Precht, H., cited................. 99 (footnote)

Pycnometer method of determining specific gravity, description of........... 29-30

Fyrite, determinations of ferrous iron as nffected by .................. 94-95

Quulitative unalyses, unadvisubility of.... 26

Fadiator for evaporation, description of ...

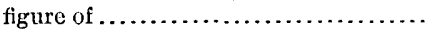

Ramsay, William, cited................ 107

Ransome and Hillebrand, cited.... $8 \$$ (footnote)

Rengents, quality of................. 25-26

Ridsdale, C. H., cited ............... 58,85

Riggs, R. B., development of Gooch amylalcohol method of separating alkalies from magnesium by........... 101

Rose, H., cited .............. 59 (footnote), 107 method of estimating fluorine devised by .......................... 103-105

Rosenbladt, T., method of estimating boron devised by ............... 107

Sandberger, F., cited ............... 21, 107, 110

Schmoeger, M., cited................... 66

Silica, method of separating alumina, etc.,

from......................... 47-54 mode of drying and testing . ......... 52-53 modes of determining............. 47-54 recovery from alumina precipitate of amounts of $56-57$
Silica, separation of, in rocks contalning

fluorine .........47,50 (fơtnote), 108

soluble, estimation of .............. 109-110

Silicates, modes of decomposition of . . .... 48-49

Silvestri, $\longrightarrow$, cited ..................... 107

Smith, J. Jawrence, figure showing crucible for alkali determinations de. vised by ...

method of determining alkalies devised

by $\ldots \ldots \ldots \ldots \ldots \ldots \ldots \ldots \ldots \ldots . \ldots 6-99$

Sodium carbonate, limit of impurity of ... 25,51 Sodium carbonate method for decomposing silicates, description of........... 50-52

Sonstadt, E., cited ............... 99 (footuote) Specific gravity, modes of determining .... 28-31 Sperry and Penfield, cited ....... 107 (footnote) Stokes, H. N., acknowledgments to....... 110 cited...................... 76,89,95 Stokes and Hillebrand, cited...... 90 (footnote) Streit and Franz, method of separating zirconium devised by ............. 76-77

Strontium, determination of............ 62-64 percentage of rock content of........ 17-18 separation of barium from.......... 63-64 separation of calcium from........... 63 Sulphides, determinations of ferrous iron as affected by ................. 94-95 methods of distinguishing ........... 105

nearly constant presence of, in rocks... 18-19 Sulphur, determination of ............ 105-106 Tantalum, rare occurrence of ........... 21 Thorium, determination of ........... 77 rare occurrence of................... 21

Titanic and phosphoric oxides, combined average of, in rocks of earth's crust.

in alumina precipitate, peculiar occurrence of ................ 57 (footnote)

Titanium, estimation of .............67-73

loss of, by evaporation with hydrofluoric acid alone............. 69 (footnote) preliminary procedure when material is scanty.

solubility in caustic alkalies of oxide of.

T'ongs for handling crucibles, description

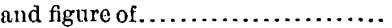

Ulbricht, $\mathbf{R}$, cited ................... 66

Ullmann, C., cited.............. $91^{\circ}$ (footnote)

Uranium, determination of............ 77

Vanadium, condition of ................ 88

determination of $\ldots \ldots \ldots \ldots \ldots \ldots \ldots \ldots .82-88$

determinations of ferrous iron as a[-

fected by .................. 94, 95,90

distribution of ................... 82

effect of, in estimation of total iron .... 58

occurrence of .................... 18

precipitation of, with iron, etc ........ 58

preliminary procedure when material

is scanty ...................... 79

qualitative tests for............... 84-85

tables showing results of tests for . .... 86,87

Vogt, J. H. I., cited .................. 89

Washington, H.S., arrangement of analyses advocated by ................... 22

Water, methods of determining ......... 32-47

Water determination, drying oven for..... 37

drying temperatures in.............. 33

figures of Jannasch's glass tubes for... $\quad 46$ 
Water determination, figures showing Gooch tubulated platinum crucible for....................... $42,43,44$ figure showing Penfield's fire-brick and charcoal oven for.

figures showing Penfield's tubes for .... 30,40

Weber and Janiasch, boric-oxide method for decomposing silicates devised

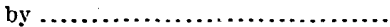

use of oxygen blast in treatment of refractory silicates by the boric-oxide method
Page.

Weller, A., colorimetric estimation of titanium by method of. ............ 67-69

Wells and Mitchell, cited ......... 57 (footnote)

Whitfield, J.E., cited ..................... 107

Wülfing, E. A.; cited ........... 89,100 (footmote)

Zinc, determination of ..................60-61

Zimmermann, C., mercuric oxife method of separuting alkalies devised by .. $\quad 100$ ric estimation of titanium......... $72-73$ estimation of ..................... 73-77 